\title{
Pion Superfluidity and Meson Properties at Finite Isospin Density
}

\author{
Lianyi He, Meng Jin, and Pengfei Zhuang \\ Physics Department, Tsinghua University, Beijing 100084, China
}

We investigate pion superfluidity and its effect on meson properties and equation of state at finite temperature and isospin and baryon densities in the frame of standard flavor SU(2) NJL model. In mean field approximation to quarks and random phase approximation to mesons, the critical isospin chemical potential for pion superfluidity is exactly the pion mass in the vacuum, and corresponding to the isospin symmetry spontaneous breaking, there is in the pion superfluidity phase a Goldstone mode which is the linear combination of the normal sigma and charged pion modes. We calculate numerically the gap equations for the chiral and pion condensates, the phase diagrams, the meson spectra, and the equation of state, and compare them with that obtained in other effective models. The competitions between pion superfluidity and color superconductivity at finite baryon density and between pion and kaon superfluidity at finite strangeness density in flavor SU(3) NJL model are briefly discussed.

PACS : 11.10.Wx, 12.38. - t, 25.75.Nq

\section{INTRODUCTION}

It is generally believed that there exists a rich phase structure of Quantum Chromodynamics (QCD) at finite temperature and baryon density, for instance, the deconfinement process from hadron gas to quark-gluon plasma, the transition from chiral symmetry breaking to the symmetry restoration [1], and the color superconductivity [2] at low temperature but high baryon density. Recently, the study on the QCD phase structure is extended to finite isospin density. The physical motivation to study QCD at finite isospin density and the corresponding pion superfluidity is related to the investigation of compact stars, isospin asymmetric nuclear matter and heavy ion collisions at intermediate energies. In early studies on dense nuclear matter and compact stars, it has been suggested that charged pions and even kaons are condensed at sufficiently high density [3-6].

While the perturbation theory of QCD can well describe the properties of the new phases at high temperature and/or high density, the study on the phase structure at moderate (baryon or/and isospin) density depends on lattice QCD calculation and effective models with QCD symmetries. While there is not yet precise lattice result at finite baryon density due to the Fermion sign problem [7], it is in principle no problem to do lattice simulation at finite isospin density [8]. Recently it is found that $[9-11]$ there is a phase transition from normal phase to pion superfluidity at a critical isospin chemical potential which is about the pion mass in the vacuum,
$\mu_{I}^{c} \simeq m_{\pi}$. The QCD phase structure at finite isospin density is also investigated in many low energy effective models, such as chiral perturbation theory [8,12-15], Nambu-Jona-Lasinio (NJL) model [16-20], random matrix method [21,22], ladder QCD [23], and strong coupling lattice QCD [24].

One of the models that enables us to see directly how the dynamic mechanisms of chiral symmetry breaking and restoration operate is the NJL model [25] applied to quarks [26-29]. The chiral phase transition line [26-32] in the temperature and baryon chemical potential $\left(T-\mu_{B}\right)$ plane calculated in the model is very close to the one obtained with lattice QCD. Recently, this model is also used to investigate the color superconductivity at moderate baryon density [33-40]. It is nature to extend the NJL model to finite isospin chemical potential $[16,17]$. In the frame of general NJL Lagrangian with $U_{A}(1)$ breaking term [19], it is analytically proved [20] at quark level that the critical isospin chemical potential for pion superfluidity is exactly the pion mass in the vacuum, $\mu_{I}^{c}=m_{\pi}$, and this relation is independent of the model parameters, regularization scheme, $U_{A}(1)$ breaking coupling, and color degrees of freedom $N_{c}$.

Unlike the effective models at hadron level where one can study the effect of phase transitions on meson and diquark properties in mean field approximation, in the NJL model applied to quarks, only the order parameters of the phase transitions, namely the condensates, can be described at mean field level, to study mesons and diquarks should go beyond the mean field approximation. It is well-known that [27] in the mean field approximation to quarks together with random phase approximation (RPA) to mesons in the NJL model, one can obtain the hadronic mass spectra and the static properties of mesons remarkably well, especially the Goldstone mode corresponding to the chiral symmetry spontaneous breaking. However, in the pion superfluidity or the color superconductivity phase, the structure of the bubble summation in RPA is much more complicated than that in the chiral breaking phase. The correlation among the mesons and diquarks becomes very important and leads to the Goldstone modes corresponding to the isospin and color symmetry spontaneous breaking. In this paper, we study analytically and numerically the pion superfluidity, its effect on the meson properties, and its possible competition with the color superconductivity and kaon condensation in the frame of standard NJL model at finite temperature and baryon, isospin and strangeness chemical potentials.

The paper is organized as follows. In Section II, we establish the mean field theory of the NJL model at finite 
temperature and baryon and isospin densities. In Section III, we study the pion superfluidity at zero temperature, zero baryon chemical potential, but finite isospin chemical potential, and calculate the chiral and pion condensates and thermodynamic functions. In Section IV, we study the temperature behavior of the pion superfluidity at zero baryon chemical potential and show the phase diagrams in the temperature and isospin chemical potential plane. In Section $\mathrm{V}$, we investigate the meson modes in both normal phase and superfluidity phase in RPA. In Section VI, the bosonized version of the $S U(2)$ NJL model is discussed at finite isospin chemical potential with pion condensation. In Section VII, we study the baryon chemical potential effect and the competition between pion and diquark condensations. In Section VIII, we briefly discuss the flavor $S U(3)$ NJL model at finite isospin and strangeness chemical potentials including both pion and kaon condensations. We summarize in Section IX.

\section{MEAN FIELD THEORY OF NJL MODEL AT FINITE $\mu_{I}$}

The standard approach for dealing with the thermodynamics of a variable particles is via the grand canonical ensemble. The key quantity for a system with baryon number conservation and isospin number conservation is the partition function defined by

$$
Z\left(T, \mu_{I}, \mu_{B}, V\right)=\operatorname{Tr} e^{-\beta\left(H-\mu_{B} B-\mu_{I} I_{3}\right)} .
$$

Here $V$ is the volume of the system, $\beta$ the inverse temperature $\beta=1 / T, \mu_{B}$ and $\mu_{I}$ are the baryon and isospin chemical potentials, and $B$ and $I_{3}$ the conserved baryon number and isospin number operators. If we take only quark field $\psi$ as elementary field of the system, the operators can be expressed as

$$
\begin{aligned}
B & =\frac{1}{N_{c}} \int d^{3} \mathbf{x} \bar{\psi} \gamma_{0} \psi \\
I_{3} & =\frac{1}{2} \int d^{3} \mathbf{x} \bar{\psi} \gamma_{0} \tau^{3} \psi
\end{aligned}
$$

The factors $1 / N_{c}$ and $1 / 2$ reflect the fact that $N_{c}$ quarks make a baryon and quark's isospin quantum number is $1 / 2$. In the imaginary time formulism of finite temperature field theory, the partition function can be represented as

$$
Z\left(T, \mu_{I}, \mu_{B}, V\right)=\int[d \bar{\psi}][d \psi] e^{\int_{0}^{\beta} d \tau \int d^{3} \mathbf{x}\left(\mathcal{L}+\bar{\psi} \hat{\mu} \gamma_{0} \psi\right)},
$$

where $\mathcal{L}$ is the Lagrangian density of the system, and $\hat{\mu}$ the quark chemical potential matrix in flavor space $\hat{\mu}=$ $\operatorname{diag}\left(\mu_{u}, \mu_{d}\right)$ with the $u$ and $d$ quark chemical potentials,

$$
\begin{aligned}
& \mu_{u}=\frac{\mu_{B}}{N_{c}}+\frac{\mu_{I}}{2} \\
& \mu_{d}=\frac{\mu_{B}}{N_{c}}-\frac{\mu_{I}}{2} .
\end{aligned}
$$

The flavor $S U(2)$ NJL Lagrangian density is defined as $[25-29]$

$$
\mathcal{L}=\bar{\psi}\left(i \gamma^{\mu} \partial_{\mu}-m_{0}\right) \psi+G\left[(\bar{\psi} \psi)^{2}+\left(\bar{\psi} i \gamma_{5} \boldsymbol{\tau} \psi\right)^{2}\right]
$$

with scalar and pseudoscalar interactions corresponding to $\sigma$ and $\pi$ excitations, or equivalently,

$$
\begin{aligned}
\mathcal{L} & =\bar{\psi}\left(i \gamma^{\mu} \partial_{\mu}-m_{0}\right) \psi \\
& +G\left[(\bar{\psi} \psi)^{2}+\left(\bar{\psi} i \gamma_{5} \tau_{3} \psi\right)^{2}+2\left(\bar{\psi} i \gamma_{5} \tau_{+} \psi\right)\left(\bar{\psi} i \gamma_{5} \tau_{-} \psi\right)\right]
\end{aligned}
$$

with $\pi_{+}$and $\pi_{-}$excitations instead of $\pi_{1}$ and $\pi_{2}$, and $\tau_{ \pm}=\left(\tau_{1} \pm i \tau_{2}\right) / \sqrt{2}$

The above Lagrangian density has the symmetry $U_{B}(1) \otimes S U_{I}(2) \otimes S U_{A}(2)$ corresponding to baryon number gauge symmetry, isospin symmetry and chiral symmetry, respectively. However, in the presence of a nonzero isospin chemical potential, the isospin symmetry $S U_{I}(2)$ breaks down to $U_{I}(1)$ global symmetry which is related to Bose-Einstein condensation of charged pions, and the chiral symmetry $S U_{A}(2)$ breaks down to $U_{I A}(1)$ global symmetry which is associated with the chiral condensation of sigma meson. Therefore, in the case with $\mu_{I} \neq 0$, chiral symmetry restoration at finite temperature and chemical potentials means only degenerate of $\sigma$ and $\pi_{0}$ meson masses. At zero baryon chemical potential, the "Fermi surfaces" of the $u(d)$ and anti- $d(u)$ quarks coincide. Therefore, at sufficiently high $\mu_{I}>0$ the condensate of $u$ and anti- $d$ quarks is favored, and at sufficiently high $\mu_{I}<0$ the condensate of $d$ and anti- $u$ quarks is favored. Since at low isospin chemical potential (and low temperature and low baryon chemical potential) there is no deconfinement, the condensates can be identified as Bose-Einstein condensates of charged pions, $\pi^{+}$ and $\pi^{-}$. Introducing the chiral condensate,

$$
\begin{aligned}
\langle\bar{\psi} \psi\rangle & =\sigma=\sigma_{u}+\sigma_{d}, \\
\sigma_{u} & =\langle\bar{u} u\rangle, \\
\sigma_{d} & =\langle\bar{d} d\rangle
\end{aligned}
$$

and two pion condensates,

$$
\begin{aligned}
& \left\langle\bar{\psi} i \gamma_{5} \tau_{+} \psi\right\rangle=\sqrt{2}\left\langle\bar{u} i \gamma_{5} d\right\rangle=\pi^{+}=\frac{\pi}{\sqrt{2}} e^{i \theta} \\
& \left\langle\bar{\psi} i \gamma_{5} \tau_{-} \psi\right\rangle=\sqrt{2}\left\langle\bar{d} i \gamma_{5} u\right\rangle=\pi^{-}=\frac{\pi}{\sqrt{2}} e^{-i \theta}
\end{aligned}
$$

a nonzero condensate $\sigma$ means spontaneous chiral symmetry breaking, and a nonzero condensate $\pi$ means spontaneous isospin symmetry breaking. The phase factor $\theta$ related to the condensates $\pi_{+}$and $\pi_{-}$indicates the direction of the $U_{I}(1)$ symmetry breaking. If the system 
is in global thermal equilibrium, $\theta$ is a constant and it does not change any physical result. For convenience we choose $\theta=0$ in the following. As is well-known and will be seen later, there are corresponding Goldstone modes in the region of spontaneous chiral breaking and the region of spontaneous isospin breaking.

Defining the meson fluctuations $\delta_{\sigma}, \delta_{\pi_{0}}, \delta_{\pi_{+}}, \delta_{\pi_{-}}$as

$$
\begin{aligned}
\bar{\psi} \psi & =\sigma+\delta_{\sigma}, \\
\bar{\psi} i \gamma_{5} \tau_{3} \psi & =\delta_{\pi_{0}}, \\
\bar{\psi} i \gamma_{5} \tau_{+} \psi & =\pi^{+}+\delta_{\pi_{+}}, \\
\bar{\psi} i \gamma_{5} \tau_{-} \psi & =\pi^{-}+\delta_{\pi_{-}},
\end{aligned}
$$

and keeping only the linear terms in $\delta$, the partition function in mean field approximation is simplified as

$$
Z\left(T, \mu_{I}, \mu_{B}, V\right)=\int[d \bar{\psi}][d \psi] e^{\int_{0}^{\beta} d \tau \int d^{3} \mathbf{x} \mathcal{L}_{m f}},
$$

with the mean field Lagrangian density

$$
\begin{aligned}
\mathcal{L}_{m f} & =\bar{\psi}\left[i \gamma^{\mu} \partial_{\mu}-\left(m_{0}-2 G \sigma\right)+\mu \gamma_{0}+2 G \pi i \gamma_{5} \tau_{1}\right] \psi \\
& -G\left(\sigma^{2}+\pi^{2}\right),
\end{aligned}
$$

from which the inverse quark propagator matrix in the flavor space as a function of quark momentum can be derived directly,

$$
\mathcal{S}_{m f}^{-1}(k)=\left(\begin{array}{cc}
\gamma^{\mu} k_{\mu}+\mu_{u} \gamma_{0}-M_{q} & 2 i G \pi \gamma_{5} \\
2 i G \pi \gamma_{5} & \gamma^{\mu} k_{\mu}+\mu_{d} \gamma_{0}-M_{q}
\end{array}\right)
$$

with the effective quark mass

$$
M_{q}=m_{0}-2 G \sigma .
$$

Using the method of massive energy projectors [34], we obtain explicitly the mean field quark propagator

$$
\mathcal{S}_{m f}(k)=\left(\begin{array}{cc}
\mathcal{S}_{u u}(k) & \mathcal{S}_{u d}(k) \\
\mathcal{S}_{d u}(k) & \mathcal{S}_{d d}(k)
\end{array}\right),
$$

with the four matrix elements

$$
\begin{aligned}
\mathcal{S}_{u u}(k) & =\frac{k_{0}+E_{k}+\mu_{d}}{\left(k_{0}-E_{-}^{-}\right)\left(k_{0}+E_{+}^{-}\right)} \Lambda_{+} \gamma_{0} \\
& +\frac{k_{0}-E_{k}+\mu_{d}}{\left(k_{0}-E_{-}^{+}\right)\left(k_{0}+E_{+}^{+}\right)} \Lambda_{-} \gamma_{0}, \\
\mathcal{S}_{d d}(k) & =\frac{k_{0}-E_{k}+\mu_{u}}{\left(k_{0}-E_{-}^{-}\right)\left(k_{0}+E_{+}^{-}\right)} \Lambda_{-} \gamma_{0} \\
& +\frac{k_{0}+E_{k}+\mu_{u}}{\left(k_{0}-E_{-}^{+}\right)\left(k_{0}+E_{+}^{+}\right)} \Lambda_{+} \gamma_{0}, \\
\mathcal{S}_{u d}(k) & =\frac{2 i G \pi}{\left(k_{0}-E_{-}^{-}\right)\left(k_{0}+E_{+}^{-}\right)} \Lambda_{+} \gamma_{5} \\
& +\frac{2 i G \pi}{\left(k_{0}-E_{-}^{+}\right)\left(k_{0}+E_{+}^{+}\right)} \Lambda_{-} \gamma_{5}, \\
\mathcal{S}_{d u}(k) & =\frac{2 i G \pi}{\left(k_{0}-E_{-}^{-}\right)\left(k_{0}+E_{+}^{-}\right)} \Lambda_{-} \gamma_{5} \\
& +\frac{2 i G \pi}{\left(k_{0}-E_{-}^{+}\right)\left(k_{0}+E_{+}^{+}\right)} \Lambda_{+} \gamma_{5},
\end{aligned}
$$

where $E_{\mp}^{ \pm}$are effective quark energies

$$
\begin{aligned}
E_{\mp}^{ \pm} & =E_{k}^{ \pm} \mp \frac{\mu_{B}}{3}, \\
E_{k}^{ \pm} & =\sqrt{\left(E_{k} \pm \mu_{I} / 2\right)^{2}+4 G^{2} \pi^{2}}, \\
E_{k} & =\sqrt{|\mathbf{k}|^{2}+M_{q}^{2}},
\end{aligned}
$$

and $\Lambda_{ \pm}$are the energy projectors

$$
\Lambda_{ \pm}(\mathbf{k})=\frac{1}{2}\left(1 \pm \frac{\gamma_{0}\left(\gamma \cdot \mathbf{k}+M_{q}\right)}{E_{k}}\right)
$$

\section{A. Gap Equations}

The quark propagator (14) is the background of calculations for quarks in mean field approximation and also for mesons in RPA. From the definitions of the chiral and pion condensates (7) and (8), it is easy to express them in terms of the matrix elements of the quark propagator,

$$
\begin{aligned}
\sigma_{u} & =-N_{c} \int \frac{d^{4} k}{(2 \pi)^{4}} \operatorname{Tr}_{D}\left[i \mathcal{S}_{u u}(k)\right], \\
\sigma_{d} & =-N_{c} \int \frac{d^{4} k}{(2 \pi)^{4}} \operatorname{Tr}_{D}\left[i \mathcal{S}_{d d}(k)\right], \\
\pi & =N_{c} \int \frac{d^{4} k}{(2 \pi)^{4}} \operatorname{Tr}_{D}\left[\left(\mathcal{S}_{u d}(k)+\mathcal{S}_{d u}(k)\right) \gamma_{5}\right],
\end{aligned}
$$

where the trace $\operatorname{Tr}_{D}$ is taken in Dirac space and the four momentum integration is defined as $\int \frac{d^{4} k}{(2 \pi)^{4}}=$ $i T \sum_{n} \int \frac{d^{3} \mathbf{k}}{(\mathbf{2} \pi)^{3}}$ at finite temperature. Obviously, in the study without considering color superconductivity, the color degrees of freedom in the NJL model is trivial, and the trace in color space simply contributes a factor $N_{c}$. Performing the trace in spin space (the trace including the energy projectors are presented in Appendix A) and the Matsubara frequency summation we have

$$
\begin{aligned}
\sigma_{u} & =\int \frac{d^{3} \mathbf{k}}{(2 \pi)^{3}} \frac{N_{c} M_{q}}{E_{k}}\left[f\left(E_{-}^{-}\right)+f\left(-E_{+}^{-}\right)-f\left(E_{-}^{+}\right)\right. \\
& -f\left(-E_{+}^{+}\right)+\frac{E_{k}-\mu_{I} / 2}{E_{k}^{-}}\left(f\left(E_{-}^{-}\right)-f\left(-E_{+}^{-}\right)\right) \\
& \left.+\frac{E_{k}+\mu_{I} / 2}{E_{k}^{+}}\left(f\left(E_{-}^{+}\right)-f\left(-E_{+}^{+}\right)\right)\right], \\
\sigma_{d} & =\int \frac{d^{3} \mathbf{k}}{(2 \pi)^{3}} \frac{N_{c} M_{q}}{E_{k}}\left[-f\left(E_{-}^{-}\right)-f\left(-E_{+}^{-}\right)+f\left(E_{-}^{+}\right)\right. \\
& +f\left(-E_{+}^{+}\right)+\frac{E_{k}-\mu_{I} / 2}{E_{k}^{-}}\left(f\left(E_{-}^{-}\right)-f\left(-E_{+}^{-}\right)\right) \\
& \left.+\frac{E_{k}+\mu_{I} / 2}{E_{k}^{+}}\left(f\left(E_{-}^{+}\right)-f\left(-E_{+}^{+}\right)\right)\right], \\
\pi & =-4 N_{c} G \pi \int \frac{d^{3} \mathbf{k}}{(2 \pi)^{3}}\left[\frac{1}{E_{k}^{-}}\left(f\left(E_{-}^{-}\right)-f\left(-E_{+}^{-}\right)\right)\right. \\
& \left.+\frac{1}{E_{k}^{+}}\left(f\left(E_{-}^{+}\right)-f\left(-E_{+}^{+}\right)\right)\right],
\end{aligned}
$$


with the Fermi-Dirac distribution function

$$
f(x)=\frac{1}{e^{\beta x}+1} .
$$

This group of gap equations has a symmetry that it is invariant under the transformations $\sigma_{u} \rightarrow \sigma_{d}, \sigma_{d} \rightarrow$ $\sigma_{u}, \mu_{I} \rightarrow-\mu_{I}$. Therefore, we can only concentrate on the case $\mu_{I}>0$ and the results for the case $\mu_{I}<0$ can be obtained easily. It is also necessary to note that for $\mu_{B}=0$ or $\mu_{I}=0$, there is always $\sigma_{u}=\sigma_{d}$, since in this case the chemical potential difference between $\bar{u}$ and $u$ is the same as the difference between $\bar{d}$ and $d$.

In any case we can combine the first two gap equations into one determining the chiral condensate $\sigma$,

$$
\begin{aligned}
\sigma & =\int \frac{d^{3} \mathbf{k}}{(2 \pi)^{3}} \frac{2 N_{c} M_{q}}{E_{k}}\left[\frac{E_{k}-\mu_{I} / 2}{E_{k}^{-}}\left(f\left(E_{-}^{-}\right)-f\left(-E_{+}^{-}\right)\right)\right. \\
& \left.+\frac{E_{k}+\mu_{I} / 2}{E_{k}^{+}}\left(f\left(E_{-}^{+}\right)-f\left(-E_{+}^{+}\right)\right)\right]
\end{aligned}
$$

We now consider the limit of $\mu_{I}=0$. In this case the gap equations are reduced to

$$
\begin{aligned}
& \sigma\left[1+\int \frac{d^{3} \mathbf{k}}{(2 \pi)^{3}} \frac{8 N_{c} G}{\sqrt{E_{k}^{2}+4 G^{2} \pi^{2}}}\left(f\left(E_{-}^{-}\right)-f\left(-E_{+}^{-}\right)\right)\right] \\
& =\int \frac{d^{3} \mathbf{k}}{(2 \pi)^{3}} \frac{4 m_{0} N_{c}}{\sqrt{E_{k}^{2}+4 G^{2} \pi^{2}}}\left(f\left(E_{-}^{+}\right)-f\left(-E_{+}^{+}\right)\right), \\
& \pi\left[1+\int \frac{d^{3} \mathbf{k}}{(2 \pi)^{3}} \frac{8 N_{c} G}{\sqrt{E_{k}^{2}+4 G^{2} \pi^{2}}}\left(f\left(E_{-}^{-}\right)-f\left(-E_{+}^{-}\right)\right)\right] \\
& =0 .
\end{aligned}
$$

Only in chiral limit, $m_{0}=0$, there is possibly nonzero $\pi$, and the gap equation

$$
\begin{aligned}
& 1+\int \frac{d^{3} \mathbf{k}}{(2 \pi)^{3}} \frac{8 N_{c} G}{\sqrt{k^{2}+4 G^{2}\left(\sigma^{2}+\pi^{2}\right)}}\left(f\left(E_{-}^{-}\right)-f\left(-E_{+}^{-}\right)\right) \\
& =0
\end{aligned}
$$

determines the combination of the two condensates $\sigma^{2}+$ $\pi^{2}$. In real world with nonzero quark mass, $m_{0} \neq 0$, the pion condensate is forced to vanish, $\pi=0$ at $\mu_{I}=0$, and $\sigma$ satisfies the well-known NJL gap equation

$$
\begin{aligned}
& \sigma-\int \frac{d^{3} \mathbf{k}}{(2 \pi)^{3}} \frac{4 N_{c} M_{q}}{E_{k}}\left[f\left(E_{k}-\frac{\mu_{B}}{3}\right)-f\left(-E_{k}-\frac{\mu_{B}}{3}\right)\right] \\
& =0 .
\end{aligned}
$$

\section{B. Thermodynamics}

The thermodynamic potential in mean field approximation

$$
\Omega=-\frac{T}{V} \ln Z=-\frac{T}{V} \operatorname{Tr} \ln \mathcal{S}_{m f}^{-1}+G\left(\sigma^{2}+\pi^{2}\right)
$$

can be evaluated with the effective quark energies

$$
\begin{aligned}
\Omega\left(T, \mu_{I}, \mu_{B} \mid \sigma_{u}, \sigma_{d}, \pi\right)=+G\left(\sigma^{2}+\pi^{2}\right) \\
\quad-N_{c} \int \frac{d^{3} \mathbf{k}}{(2 \pi)^{3}}\left[E_{-}^{-}-E_{+}^{-}+E_{-}^{+}-E_{+}^{+}\right. \\
+2 T\left(\ln \left(1+e^{-E_{-}^{-} / T}\right)+\ln \left(1+e^{E_{+}^{-} / T}\right)\right. \\
\left.\left.+\ln \left(1+e^{-E_{-}^{+} / T}\right)+\ln \left(1+e^{E_{+}^{+} / T}\right)\right)\right] .
\end{aligned}
$$

The gap equations (19) for the condensates are equivalent to the extremum condition of the thermodynamic potential,

$$
\frac{\partial \Omega}{\partial \sigma_{u}}=0, \quad \frac{\partial \Omega}{\partial \sigma_{d}}=0, \quad \frac{\partial \Omega}{\partial \pi}=0 .
$$

Note that when there exist multi roots of the gap equations, only the solution which satisfies the minimum condition

$$
\frac{\partial^{2} \Omega}{\partial \sigma_{u}^{2}} \geq 0, \quad \frac{\partial^{2} \Omega}{\partial \sigma_{d}^{2}} \geq 0, \quad \frac{\partial^{2} \Omega}{\partial \pi^{2}} \geq 0
$$

is physical.

Once $\Omega$ is known, the thermodynamic functions that measure the bulk properties of matter can be obtained. For an infinite system, these are the pressure $p$, the entropy density $s$, the charge number densities $n_{B}$ and $n_{I}$, the flavor number densities $n_{u}$ and $n_{d}$, the energy density $\epsilon$, and the specific heat $c$, that are defined as

$$
\begin{aligned}
p & =-\Omega, \\
s & =-\frac{\partial \Omega}{\partial T}, \\
n_{B} & =-\frac{\partial \Omega}{\partial \mu_{B}}, \quad n_{I}=-\frac{\partial \Omega}{\partial \mu_{I}}, \\
n_{u} & =-\frac{\partial \Omega}{\partial \mu_{u}}, \quad n_{d}=-\frac{\partial \Omega}{\partial \mu_{d}}, \\
\epsilon & =-p+T s+\mu_{I} n_{I}+\mu_{B} n_{B}, \\
c & =\frac{\partial \epsilon}{\partial T} .
\end{aligned}
$$

The flavor number densities $n_{u}=\left\langle\bar{u} \gamma_{0} u\right\rangle$ and $n_{d}=$ $\left\langle\bar{d} \gamma_{0} d\right\rangle$ can also be obtained directly from the matrix elements of the quark propagator,

$$
\begin{aligned}
n_{u} & =-N_{c} \int \frac{d^{4} k}{(2 \pi)^{4}} \operatorname{Tr}_{D}\left[i \mathcal{S}_{u u}(k) \gamma_{0}\right] \\
& =N_{c} \int \frac{d^{3} \mathbf{k}}{(2 \pi)^{3}}\left[f\left(E_{-}^{-}\right)+f\left(-E_{+}^{-}\right)+f\left(E_{-}^{+}\right)\right. \\
& +f\left(-E_{+}^{+}\right)+\frac{E_{k}-\mu_{I} / 2}{E_{k}^{-}}\left(f\left(E_{-}^{-}\right)-f\left(-E_{+}^{-}\right)\right) \\
& \left.-\frac{E_{k}+\mu_{I} / 2}{E_{k}^{+}}\left(f\left(E_{-}^{+}\right)-f\left(-E_{+}^{+}\right)\right)-2\right] \\
n_{d} & =-N_{c} \int \frac{d^{4} k}{(2 \pi)^{4}} \operatorname{Tr}_{D}\left[i \mathcal{S}_{d d}(k) \gamma_{0}\right]
\end{aligned}
$$




$$
\begin{aligned}
& =N_{c} \int \frac{d^{3} \mathbf{k}}{(2 \pi)^{3}}\left[f\left(E_{-}^{-}\right)+f\left(-E_{+}^{-}\right)+f\left(E_{-}^{+}\right)\right. \\
& +f\left(-E_{+}^{+}\right)-\frac{E_{k}-\mu_{I} / 2}{E_{k}^{-}}\left(f\left(E_{-}^{-}\right)-f\left(-E_{+}^{-}\right)\right) \\
& \left.+\frac{E_{k}+\mu_{I} / 2}{E_{k}^{+}}\left(f\left(E_{-}^{+}\right)-f\left(-E_{+}^{+}\right)\right)-2\right]
\end{aligned}
$$

Each pure number density is the difference between the corresponding quark number density and antiquark number density,

$$
\begin{aligned}
& n_{u}=n_{u}^{+}-n_{u}^{-}, \\
& n_{d}=n_{d}^{+}-n_{d}^{-} .
\end{aligned}
$$

With the help of the positive and negative energy projectors $\Lambda_{+}$and $\Lambda_{-}$, we separate $n_{u, d}^{ \pm}$from $n_{u, d}$,

$$
\begin{aligned}
n_{u}^{+} & =N_{c} \int \frac{d^{3} \mathbf{k}}{(2 \pi)^{3}}\left[f\left(E_{-}^{-}\right)+f\left(-E_{+}^{-}\right)\right. \\
& \left.+\frac{E_{k}-\mu_{I} / 2}{E_{k}^{-}}\left(f\left(E_{-}^{-}\right)-f\left(-E_{+}^{-}\right)\right)\right], \\
n_{u}^{-} & =-N_{c} \int \frac{d^{3} \mathbf{k}}{(2 \pi)^{3}}\left[f\left(E_{-}^{+}\right)+f\left(-E_{+}^{+}\right)\right. \\
& \left.-\frac{E_{k}+\mu_{I} / 2}{E_{k}^{+}}\left(f\left(E_{-}^{+}\right)-f\left(-E_{+}^{+}\right)\right)-2\right], \\
n_{d}^{+} & =N_{c} \int \frac{d^{3} \mathbf{k}}{(2 \pi)^{3}}\left[f\left(E_{-}^{+}\right)+f\left(-E_{+}^{+}\right)\right. \\
& \left.+\frac{E_{k}+\mu_{I} / 2}{E_{k}^{+}}\left(f\left(E_{-}^{+}\right)-f\left(-E_{+}^{+}\right)\right)\right], \\
n_{d}^{-} & =-N_{c} \int \frac{d^{3} \mathbf{k}}{(2 \pi)^{3}}\left[f\left(E_{-}^{-}\right)+f\left(-E_{+}^{-}\right)\right. \\
& \left.-\frac{E_{k}-\mu_{I} / 2}{E_{k}^{-}}\left(f\left(E_{-}^{-}\right)-f\left(-E_{+}^{-}\right)\right)-2\right] .
\end{aligned}
$$

It is easy to obtain the relations between the flavor number densities and the charge number densities,

$$
\begin{aligned}
n_{I} & =\frac{1}{2}\left(n_{u}-n_{d}\right), \\
n_{B} & =\frac{1}{3}\left(n_{u}+n_{d}\right) .
\end{aligned}
$$

\section{PURE ISOSPIN EFFECT IN MEAN FIELD APPROXIMATION}

To solve the gap equations and calculate the thermodynamic functions numerically, we should first fix the model parameters. Because of the contact interaction between quarks that is introduced in the model, there is of course no confinement. A further consequence of this feature is that the model is non-renormalizable, and it is necessary to introduce a regulator $\Lambda$ that serves as a length scale in the problem, and which can be thought of as indicating the onset of asymptotic freedom [41]. In the following we take a hard three-momentum cutoff $\Lambda$, which and the other two model parameters, the effective coupling constant $G$ and the current quark mass $m_{0}$, can be fixed by fitting the pion mass $m_{\pi}=0.134 \mathrm{GeV}$, the pion decay constant $f_{\pi}=0.093 \mathrm{GeV}$, and the quark condensate density $\sigma_{u}=\sigma_{d}=(-0.25 \mathrm{GeV})^{3}$ in the vacuum. They are [31] $m_{0}=0, \Lambda=0.65 \mathrm{GeV}$ and $G=5.01 \mathrm{GeV}^{-2}$ in the chiral limit, and $m_{0}=0.005 \mathrm{GeV}, \Lambda=0.653 \mathrm{GeV}$, and $G=4.93 \mathrm{GeV}^{-2}$ in the real word.

In this section we concentrate on the pure isospin effect with $T=\mu_{B}=0$. In this case the difference between the $u$ quark and $d$ quark condensates disappears and we need to calculate the total chiral condensate $\sigma=2 \sigma_{u}=2 \sigma_{d}$ only.

\section{A. Pion and Chiral Condensates}

At zero temperature and baryon chemical potential the gap equations for the chiral and pion condensates are reduced to

$$
\begin{aligned}
& \sigma+2 N_{c} M_{q} \int \frac{d^{3} \mathbf{k}}{(2 \pi)^{3}} \frac{1}{E_{k}}\left(\frac{E_{k}-\mu_{I} / 2}{E_{k}^{-}}+\frac{E_{k}+\mu_{I} / 2}{E_{k}^{+}}\right)=0 \\
& \pi\left[1-4 N_{c} G \int \frac{d^{3} \mathbf{k}}{(2 \pi)^{3}}\left(\frac{1}{E_{k}^{-}}+\frac{1}{E_{k}^{+}}\right)\right]=0 .
\end{aligned}
$$

It is clear that the order parameter $\pi=0$ is always a solution of the second gap equation, it corresponds to the phase with isospin symmetry at low isospin chemical potential. The other order parameter $\sigma$ describing chiral properties in this phase is $\mu_{I}$ independent,

$$
\sigma+4 N_{c} M_{q} \int \frac{d^{3} \mathbf{k}}{(2 \pi)^{3}} \frac{1}{E_{k}}=0 .
$$

This means that the chiral condensate keeps its vacuum value in the isospin symmetric phase with $\pi=0$.

At the critical isospin chemical potential $\mu_{I}^{c}$ where the isospin symmetry starts to break spontaneously and the pion condensate appears, the solution $\pi=0$ should satisfies the equation

$$
1-8 N_{c} G \int \frac{d^{3} \mathbf{k}}{(2 \pi)^{3}} \frac{1}{E_{k}} \frac{E_{k}^{2}}{E_{k}^{2}-\left(\mu_{I}^{c}\right)^{2} / 4}=0 .
$$

From the comparison with the well-known pole equation [26-31] determining the pion mass in the vacuum,

$$
1-8 N_{c} G \int \frac{d^{3} \mathbf{k}}{(2 \pi)^{3}} \frac{1}{E_{k}} \frac{E_{k}^{2}}{E_{k}^{2}-m_{\pi}^{2} / 4}=0,
$$

where the quark mass $M_{q}$ hidden in the quark energy $E_{k}$ is the same as that in (36) and controlled by the gap equation (35), we find explicitly that the critical isospin 
chemical potential $\mu_{I}^{c}$ for the pion condensation phase transition at $T=\mu_{B}=0$ is exactly equal to the pion mass in the vacuum,

$$
\mu_{I}^{c}=m_{\pi} .
$$

For $\mu_{I}>\mu_{I}^{c}$ the isospin dependence of the two order parameters is governed by the first equation of (34) and

$$
1-4 N_{c} G \int \frac{d^{3} \mathbf{k}}{(2 \pi)^{3}}\left(\frac{1}{E_{k}^{-}}+\frac{1}{E_{k}^{+}}\right)=0 .
$$

The condensates $\sigma$ and $\pi$ scaled by the chiral condensate $\sigma_{0}$ in the vacuum are shown in Fig.(1) as functions of $\mu_{I}$. In chiral limit, pions are Goldstone particles of chiral symmetry spontaneous breaking, $m_{\pi}=0$. Therefore, at $\mu_{I}=0$ the pion condensate jumps up from the vacuum value $\pi=0$ to a finite value, and the chiral condensate jumps down from the vacuum value $\sigma_{0}=2(-0.25 \mathrm{GeV})^{3}$ to zero and then keeps zero in the region $\mu_{I}>0$ due to the constraint of the gap equations (34). These sudden changes at the critical point $\mu_{I}^{c}=0$ means that in chiral limit, no matter how small the isospin chemical potential is, the chiral symmetry is restored and pion Bose-Einstein condensate occurs. In the real world with nonzero current quark mass, the critical point is at $\mu_{I}^{c}=m_{\pi}=0.134$ $\mathrm{GeV}$ and the both chiral and pion superfluidity phase transitions are of second order. When the isospin chemical potential is small, the vacuum state is not disturbed. Only when it exceeds the pion mass, the pion condensate goes up with $\mu_{I}$ and at the same time the chiral condensate drops down with $\mu_{I}$. The two condensates coexist in a wide region. Different from the result obtained with chiral perturbation theory [8] where the pion condensate at finite $\mu_{I}$ is always less than the chiral condensate in the vacuum, $\pi / \sigma_{0}<1, \pi$ in our calculation can be larger than $\sigma_{0}$ at some intermediate isospin chemical potential. The pion condensate tends to zero at about $\mu_{I}=1.75 \mathrm{GeV}$. While the value of the endpoint of pion condensation in our calculation is closely related to the finite momentum cutoff $\Lambda$, the disappearance of pion condensation at sufficiently high isospin chemical potential can be understood in the frame of asymptotic freedom of QCD.

What is the isospin effect on the chiral condensate if we do not consider isospin spontaneous breaking? In this case, the only condensate, the chiral condensate $\sigma$ is controlled by the gap equation

$$
\sigma+4 N_{c} M_{q} \int \frac{d^{3} \mathbf{k}}{(2 \pi)^{3}} \frac{1}{E_{k}} \Theta\left(E_{k}-\left|\mu_{I}\right| / 2\right)=0,
$$

the isospin chemical potential dependence of $\sigma$ is similar to its well-known baryon chemical potential dependence. In chiral limit, the phase transition point is at

$$
\mu_{I}=2 \sqrt{\Lambda^{2}-\frac{\pi^{2}}{2 G N_{c}}} .
$$
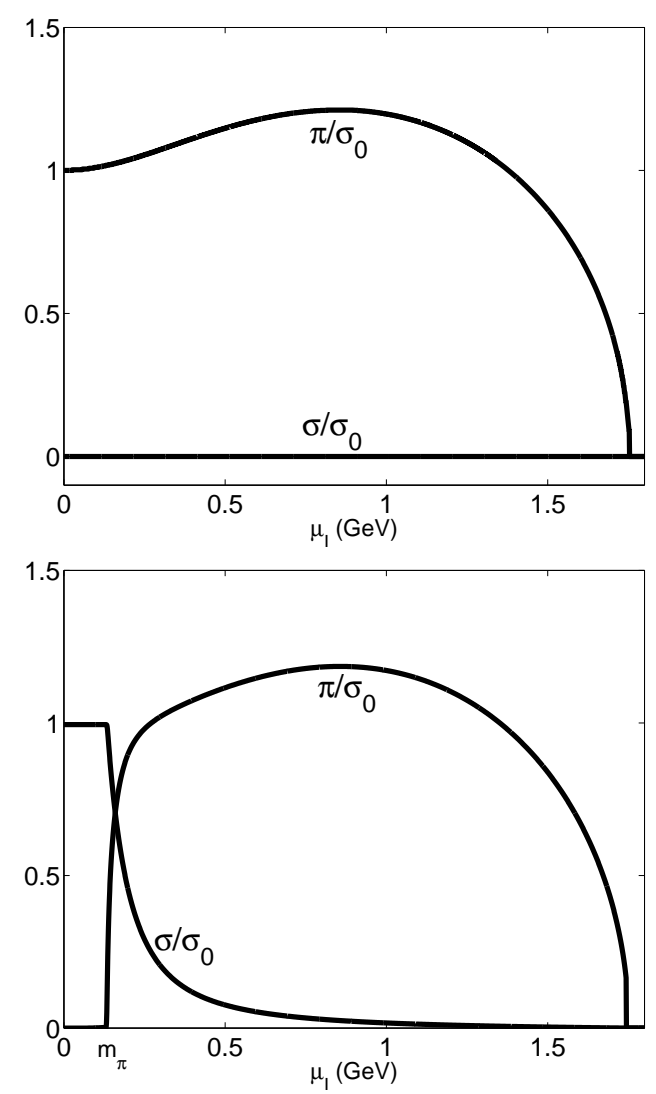

FIG. 1. The chiral and pion condensates $\sigma$ and $\pi$, scaled by the chiral condensate $\sigma_{0}$, as function of isospin chemical potential $\mu_{I}$ in the chiral limit (upper panel) and real world (lower panel) at $T=\mu_{B}=0$.

\section{B. Dispersion Relations}

The dispersion relations of quasi-particles are given by the poles of the quark propagator (14),

$$
\begin{array}{ll}
\omega_{1}(|\mathbf{k}|)=E_{-}^{-}(|\mathbf{k}|), & \omega_{2}(|\mathbf{k}|)=-E_{+}^{-}(|\mathbf{k}|) \\
\omega_{3}(|\mathbf{k}|)=E_{-}^{+}(|\mathbf{k}|), & \omega_{4}(|\mathbf{k}|)=-E_{+}^{+}(|\mathbf{k}|) .
\end{array}
$$

In the case with $\mu_{B}=0$, it is easy to see the symmetry of $\omega_{1}=-\omega_{2}$ and $\omega_{3}=-\omega_{4}$. The dispersion relations in this case are shown in Fig.(2) at $T=0$. In both the isospin symmetric phase with $\mu_{I}=0.1 \mathrm{GeV}<m_{\pi}$ and the symmetry breaking phase with $\mu_{I}=0.4 \mathrm{GeV}$ $>m_{\pi}$, any quasi-particle excitation needs energy. In the normal phase with $\pi=0$, the chiral condensate $\sigma$ opens the gap for the quasi-particle excitations, and in the pion superfluidity phase with $\mu_{I}>m_{\pi}$, the gap is mainly due to the pion condensate $\pi \neq 0$. In the normal phase, the minima of the quasi-particle energies are located at $|\mathbf{k}|=0$, but in the superfluidity phase, the location of the minima of $\omega_{1}$ and $\omega_{2}$ are shifted to a finite momentum when $\mu_{I}$ is large enough. 

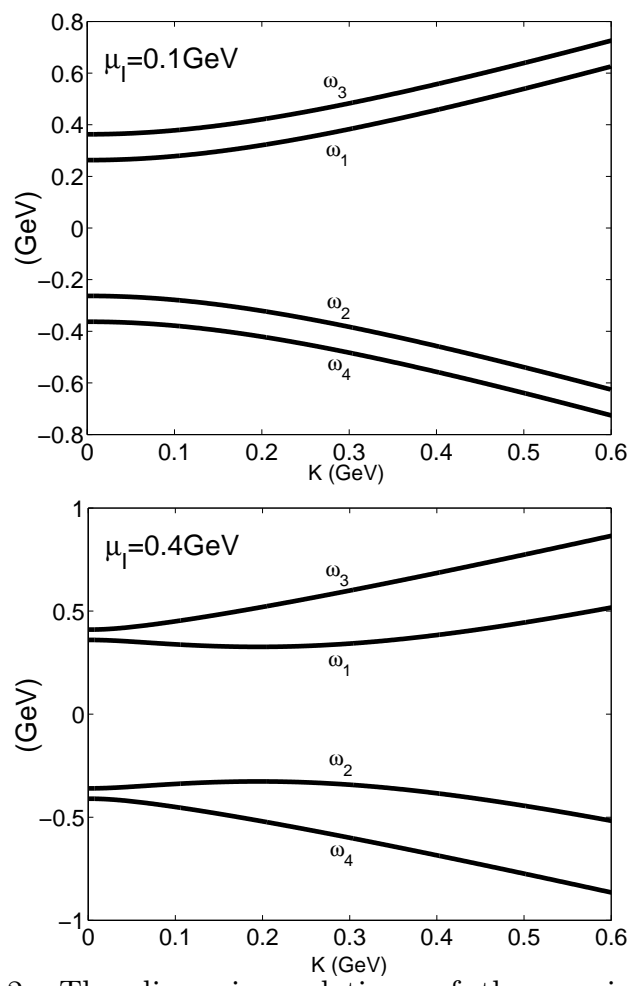

FIG. 2. The dispersion relations of the quasi-particles $\omega_{i}, i=1,2,3,4$ in the normal phase with $\mu_{I}=0.1 \mathrm{GeV}$ (upper panel) and pion superfluidity phase with $\mu_{I}=0.4 \mathrm{GeV}$ (lower panel) at $T=\mu_{B}=0$.

The quark occupation numbers $n_{u}^{+}(|\mathbf{k}|), \quad n_{u}^{-}(|\mathbf{k}|)$, $n_{d}^{+}(|\mathbf{k}|)$ and $n_{d}^{-}(|\mathbf{k}|)$ in momentum space are just the integrated functions in (32). At $T=\mu_{B}=0$ they are reduced to

$$
\begin{aligned}
& n_{u}^{+}(|\mathbf{k}|)=n_{d}^{-}(|\mathbf{k}|)=1-\frac{E_{k}-\mu_{I} / 2}{E_{k}^{-}}, \\
& n_{d}^{+}(|\mathbf{k}|)=n_{u}^{-}(|\mathbf{k}|)=1-\frac{E_{k}+\mu_{I} / 2}{E_{k}^{-}} .
\end{aligned}
$$

In the normal phase we have $n_{u}^{+}(|\mathbf{k}|)=n_{u}^{-}(|\mathbf{k}|)=$ $n_{d}^{+}(|\mathbf{k}|)=n_{d}^{-}(|\mathbf{k}|)=0$, since the ground state in this case is just the vacuum state and there are no quark and anti-quark excitations. In the superfluidity phase, there are excited quarks and anti-quarks, the occupation numbers are shown in Fig.(3) as functions of momentum. Note that the maximal occupation number is 2 because of the spin degenerate.

\section{Bulk Properties}

We first calculate the isospin density. At $T=\mu_{B}=0$ it is

$$
n_{I}=N_{c} \int \frac{d^{3} \mathbf{k}}{(2 \pi)^{3}}\left[\frac{E_{k}+\mu_{I} / 2}{E_{k}^{+}}-\frac{E_{k}-\mu_{I} / 2}{E_{k}^{-}}\right],
$$

the numerical result, scaled by the normal nuclear density $n_{0}=0.17 / \mathrm{fm}^{3}$, is shown in Fig.(4). Again in the normal phase with $\mu_{I}<m_{\pi}$ the unchanged ground state leads to a zero isospin density. Only when $\mu_{I}$ exceeds the vacuum pion mass, $n_{I}$ increases monotonously with $\mu_{I}$. This nonzero net isospin density is due to the BoseEinstein condensation of charged pions which results in different flavor densities. To see this clearly, we plot the flavor densities, scaled by the normal nuclear density $n_{0}$, as functions of isospin chemical potential in Fig.(5). In the superfluidity phase the relation $n_{u}^{+}=n_{d}^{-}>n_{d}^{+}=n_{u}^{-}$ leads to the net isospin density, and then the number of $\pi_{+}$in the system should be larger than the number of $\pi_{-}$.
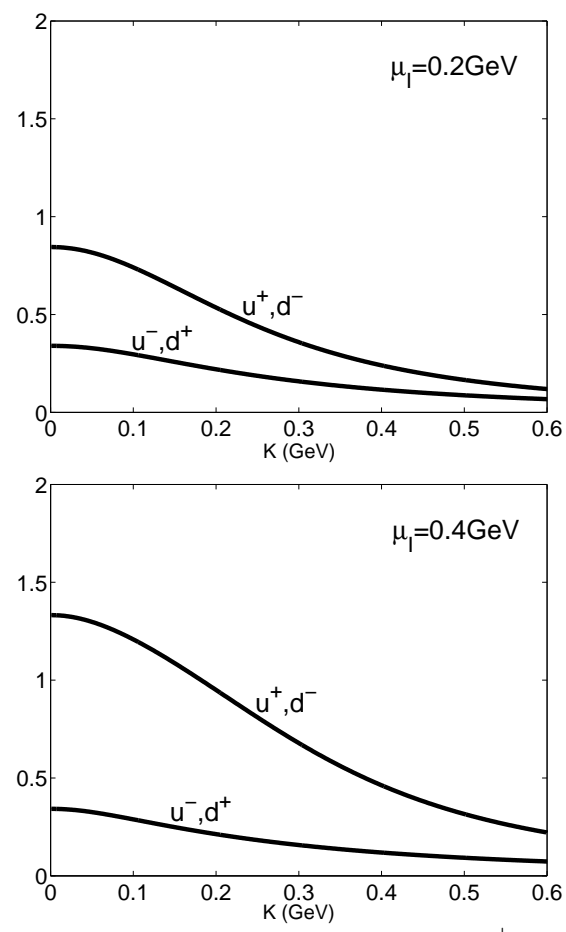

FIG. 3. The quark occupation numbers $n_{u, d}^{ \pm}(|\mathbf{k}|)$ as functions of momentum in pion superfluidity phase with $\mu_{I}=0.2$ $\mathrm{GeV}$ (upper panel) and $\mu_{I}=0.4 \mathrm{GeV}$ (lower panel) at $T=\mu_{B}=0$.

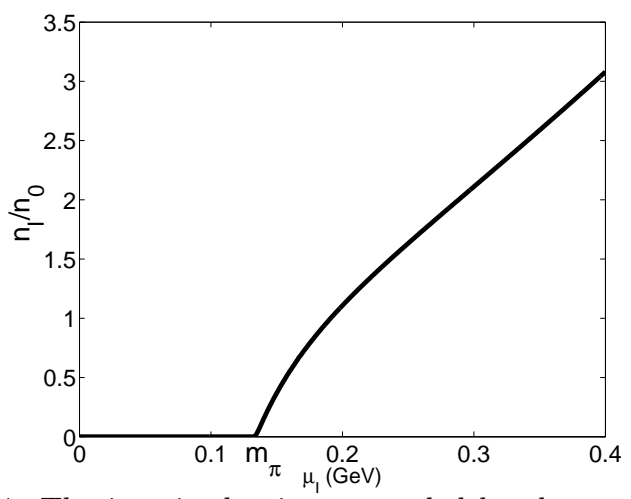

FIG. 4. The isospin density $n_{I}$, scaled by the normal nuclear density $n_{0}$, as a function of $\mu_{I}$ at $T=\mu_{B}=0$. 


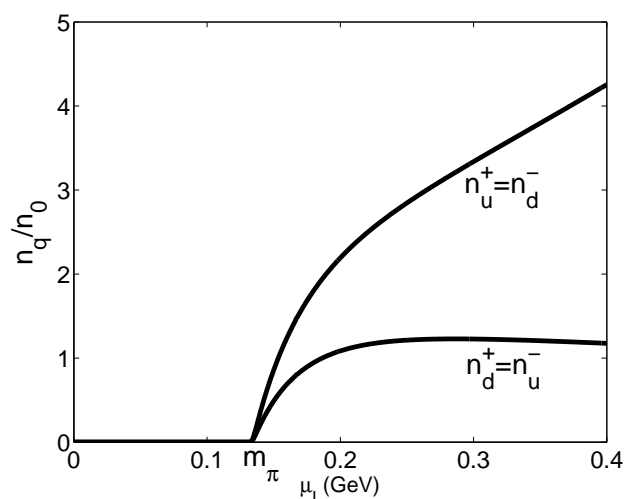

FIG. 5. The flavor densities $n_{u, d}^{ \pm}$, scaled by the normal nuclear density $n_{0}$, as functions of $\mu_{I}$ at $T=\mu_{B}=0$.

Physically, the thermodynamic potential $\Omega\left(T, \mu_{I}, \mu_{B}, \mid \sigma_{u}\left(T, \mu_{I}, \mu_{B}\right), \sigma_{d}\left(T, \mu_{I}, \mu_{B}\right), \pi\left(T, \mu_{I}, \mu_{B}\right)\right)$ corresponds to the pressure except for a sign, and only the pressure relative to the physical vacuum $\Omega_{v a c}^{p h y s}$ can be measured. The physical vacuum is defined to be

$$
\Omega_{v a c}^{\text {phys }}=\Omega\left(0,0,0 \mid \sigma_{u}(0,0,0), \sigma_{d}(0,0,0), \pi(0,0,0)\right),
$$

which for the mean field approximation corresponds to

$$
\left(\Omega_{v a c}^{\text {phys }}\right)_{m f}=\frac{\left(M_{q}(0,0,0)-m_{0}\right)^{2}}{4 G}-4 N_{c} \int \frac{d^{3} \mathbf{k}}{(2 \pi)^{3}} E_{k} .
$$

We introduce the rescaled thermodynamic potential

$$
\bar{\Omega}\left(T, \mu_{I}, \mu_{B} \mid \sigma_{u}, \sigma_{d}, \pi\right)=\Omega\left(T, \mu_{I}, \mu_{B} \mid \sigma_{u}, \sigma_{d}, \pi\right)-\Omega_{v a c}^{p h y s},
$$

the measurable pressure $p$ and the energy density $\epsilon$ are related to it. Of course, the redefinition of $\Omega$ will not change the number densities and the entropy density since they are the derivatives of $\Omega$ with respect to $\mu$ and $T$.

The pressure and energy density are shown in Fig.(6) at $T=\mu_{B}=0$. From the redefinition (47) they are zero in the vacuum and keep zero in the normal phase, since the isospin effect is not strong enough to disturb the vacuum state. In the pion superfluidity phase, the ratio of $p$ to $\epsilon$ which describes the equation of state of the system goes up with increasing $\mu_{I}$ first and then gets saturated, $p / \epsilon \sim 0.7$, at $\mu_{I} \sim 0.5 \mathrm{GeV}$. This behavior indicates that the system is far from an ideal gas which satisfies the equation of state $p=\frac{1}{3} \epsilon$.

To conclude this subsection, we remark that the isospin number density $n_{I}$ is perhaps a more physical variable than the isospin chemical potential $\mu_{I}$, since $n_{I}$ can be directly measured. The chiral and pion condensates $\sigma$ and $\pi$, scaled by the chiral condensate $\sigma_{0}$ in the vacuum, the pressure $p$ and energy density $\epsilon$, and the ratio of $p$ to $\epsilon$ are shown again in Figs.(7) and (8). Different from their $\mu_{I}$-dependence, Figs.(1) and (6), where the isospin symmetric and symmetry breaking phases are shown simultaneously and are separated from each other at $\mu_{I}=m_{\pi}$, the isospin symmetric phase is now contracted at a point $n_{I}=0$. The isospin density corresponding to the saturated equation of state is about 5 times the normal nuclear density.
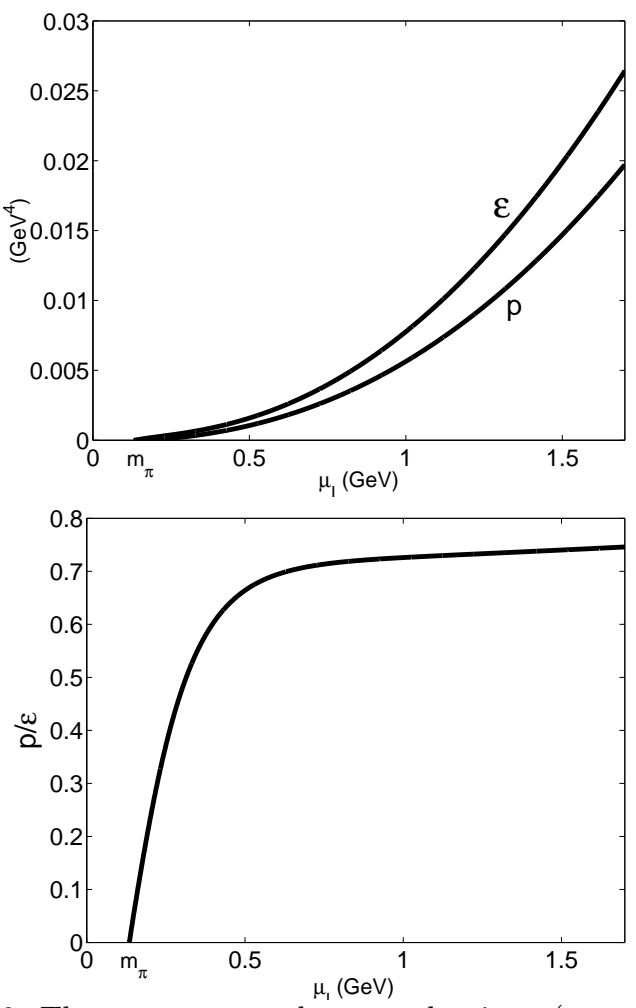

FIG. 6. The pressure $p$ and energy density $\epsilon$ (upper panel) and the ratio of $p$ to $\epsilon$ (lower panel) as functions of $\mu_{I}$ at $T=\mu_{B}=0$.

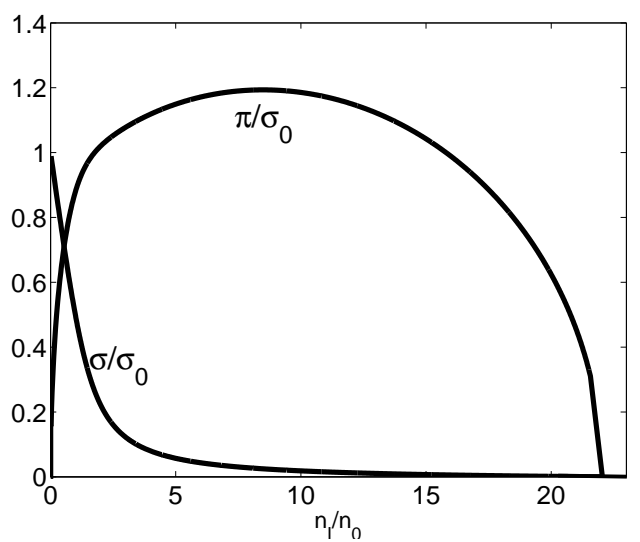

FIG. 7. The chiral and pion condensates $\sigma$ and $\pi$, scaled by the chiral condensate $\sigma_{0}$ in the vacuum, as functions of isospin density $n_{I}$ at $T=\mu_{B}=0$. 

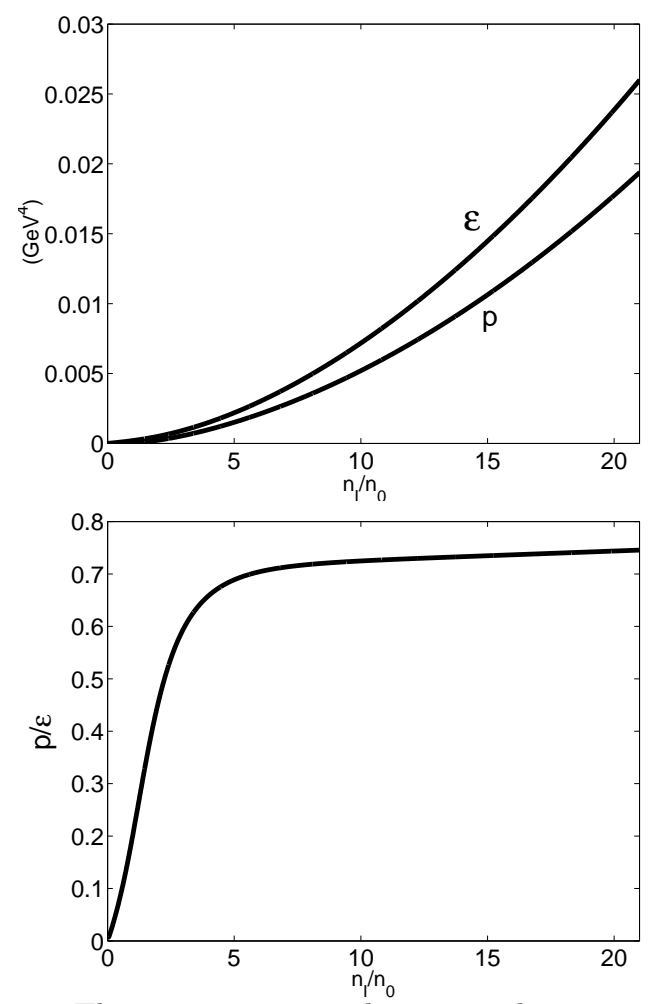

FIG. 8. The pressure $p$ and energy density $\epsilon$ (upper panel)and the ratio of $p$ to $\epsilon$ (lower panel) as functions of isospin density $n_{I}$ at $T=\mu_{B}=0$.

\section{TEMPERATURE EFFECT IN MEAN FIELD APPROXIMATION}

We now study the temperature behavior of the chiral and pion condensates and the thermodynamic functions, and discuss the phase diagram in the $T-\mu_{I}$ plane in mean field approximation at $\mu_{B}=0$.

\section{A. Chiral and Pion Condensates}

The temperature dependence of the two condensates $\sigma$ and $\pi$, again scaled by the chiral condensate $\sigma_{0}$ in the vacuum, at a fixed isospin chemical potential $\mu_{I}=0.05$ $\mathrm{GeV}$ is shown in Fig.(9) in the chiral limit. In the chiral limit any small isospin chemical potential will force the broken chiral symmetry to be restored and the isospin symmetry to be broken at $T=0$. Therefore, the chiral condensate keeps zero, and the pion condensate is continuously melted in the hot medium. Finally at a critical temperature $T_{c} \sim 0.18 \mathrm{GeV}$ the pion condensate vanishes and the isospin symmetry is restored. At small isospin chemical potential the value of the critical temperature for isospin restoration is almost the same for chiral restoration in the study without considering pion condensation $[27,31]$. In Fig.(10) we show the pion and chiral condensates as functions of $T$ at $\mu_{I}=0.15 \mathrm{GeV}$ and $0.2 \mathrm{GeV}$. For $\mu_{I}>m_{\pi}$, the pion condensate is already nonzero at the beginning, then drops down due to the temperature effect, and finally disappears at a critical temperature $T_{c}$. Like the standard BCS theory, the critical temperature and the pion condensate at $T=0$ satisfies the linear relation,

$$
T_{C}\left(\mu_{I}\right) \sim 0.57 \pi\left(T=0, \mu_{I}\right) .
$$

The relative strength of the two condensates at the beginning depends on the isospin chemical potential, $\sigma(T=$ $0)>\pi(T=0)$ at small $\mu_{I}$ and $\pi(T=0)>\sigma(T=0)$ at large $\mu_{I}$. Qualitatively different from the study on chiral symmetry restoration without considering pion condensation where the chiral condensate decreases with increasing temperature monotonously, here $\sigma$ goes up continuously in the coexistence region of the two condensates, and drops down only in the region where the isospin symmetry is restored. However, we found that when $\mu_{I}$ is close to the critical value $\mu_{I}^{c}=m_{\pi}$, say $m_{\pi}<\mu_{I}<0.2 \mathrm{GeV}$, the total condensate $\sqrt{\sigma^{2}+\pi^{2}}$ (the dashed line in Fig.(10), behaviors in a similar way like the $\sigma$ in the normal study without considering pion condensation.

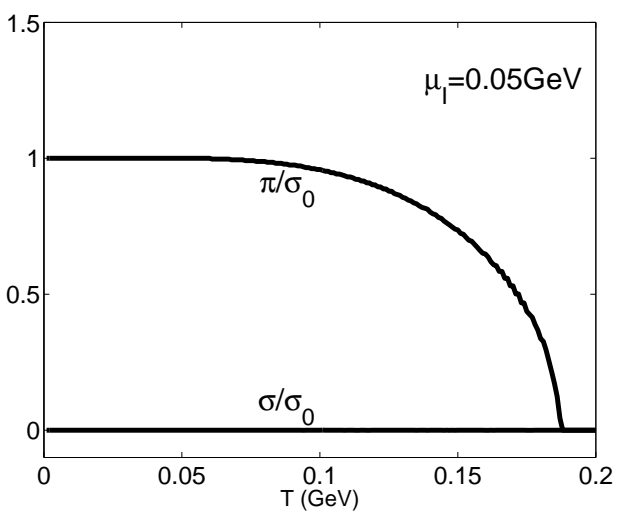

FIG. 9. The chiral and pion condensates $\sigma$ and $\pi$, scaled by the chiral condensate $\sigma_{0}$ in the vacuum, as functions of temperature $T$ at $\mu_{B}=0$ and $\mu_{I}=0.05 \mathrm{GeV}$ in the chiral limit.

\section{B. Number Densities}

The isospin density $n_{I}$ and flavor densities $n_{u, d}^{ \pm}$, scaled by the normal nuclear density $n_{0}$, are shown in Fig.(11) as functions of isospin chemical potential $\mu_{I}$ at $\mu_{B}=0$ but $T=0.1 \mathrm{GeV}$. At finite temperature, there is thermal excitation of particles. Since $T=0.1 \mathrm{GeV}$ is not very high, the critical isospin chemical potential $\mu_{I}^{c}$ for pion condensation is still approximately the vacuum pion mass $m_{\pi}$. In the region of $\mu_{I}<\mu_{I}^{c}$, the small but finite isospin density is purely due to the thermal excitation, while in the region of $\mu_{I}>\mu_{I}^{c}$ the rapidly increasing density is dominated by the Bose-Einstein condensation of charged pions. The thermal excitation depends strongly on the 
isospin chemical potential carried by the particle. At $\mu_{I}<\mu_{I}^{c}$, the densities of quarks with positive isospin chemical potential increase with $\mu_{I}$, while the densities of quarks with negative isospin chemical potential decrease with $\mu_{I}$.
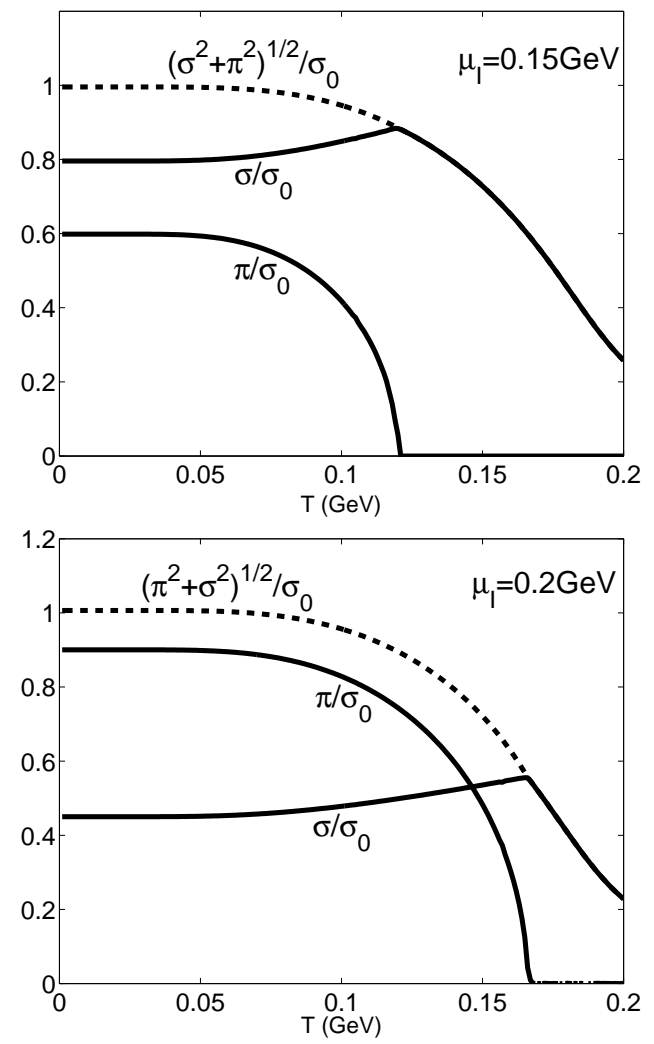

FIG. 10. The chiral and pion condensates $\sigma$ and $\pi$, scaled by the chiral condensate $\sigma_{0}$ in the vacuum, as functions of temperature $T$ at $\mu_{B}=0$ and $\mu_{I}=0.15 \mathrm{GeV}$ (upper panel) and $\mu_{I}=0.2 \mathrm{GeV}$ (lower panel) in the real world. The dashed lines are for the total condensate $\left(\sigma^{2}+\pi^{2}\right)^{1 / 2} / \sigma_{0}$.

\section{Phase Diagram in $T-\mu_{I}$ Plane}

In the chiral limit when $m_{0}=0$, the solution of the last gap equation of (19) for $\pi$ with $\sigma_{u}=\sigma_{d}=0$ separates the region of isospin symmetry breaking with $\pi \neq 0$ from the region of symmetry restoration with $\pi=0$. The phase transition line demarcating these two regions is given in the upper panel of Fig.(12) in the $T-\mu_{I}$ plane for $\mu_{B}=0$. Since an infinite small isospin effect can restore the chiral symmetry, there is $\sigma=0$ in the whole plane in the chiral limit. In the real world with nonzero current quark mass, the system is in isospin symmetric phase not only at high temperature and/or high isospin chemical potential but also at low isospin chemical potential with $\mu_{I} \leq m_{\pi}$. Since the chiral symmetry phase transition is not well defined in the case with nonzero current quark mass, we did not show the chiral phase transition line. In principle, there is $\sigma \neq 0$ in the whole $T-\mu_{I}$ plane.
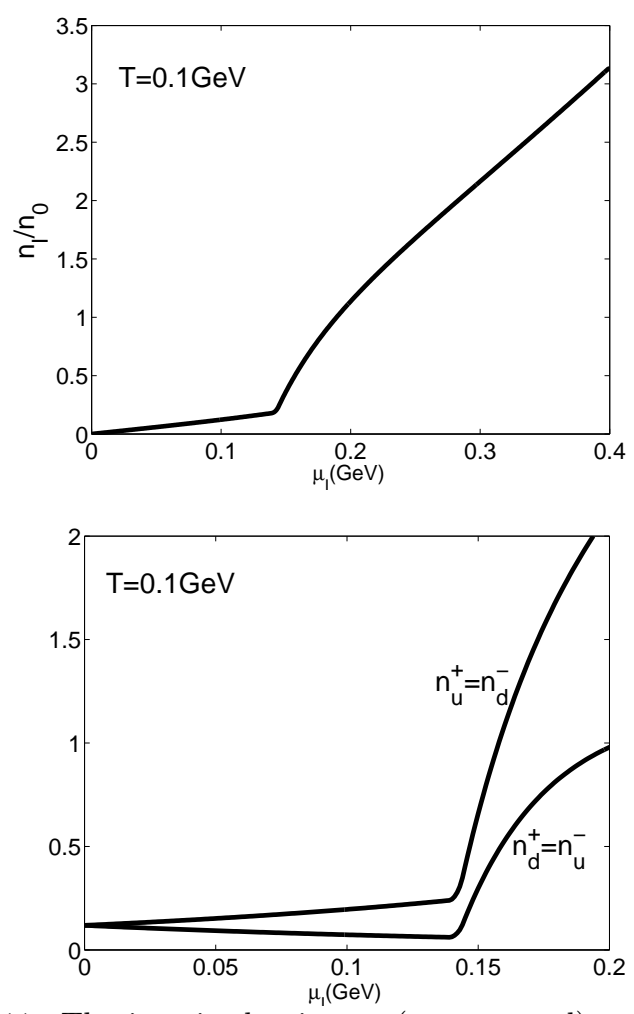

FIG. 11. The isospin density $n_{I}$ (upper panel) and flavor densities $n_{u, d}^{ \pm}$(lower panel), scaled by the normal nuclear density $n_{0}$, as functions of isospin chemical potential $\mu_{I}$ at $\mu_{B}=0$ and $T=0.1 \mathrm{GeV}$.
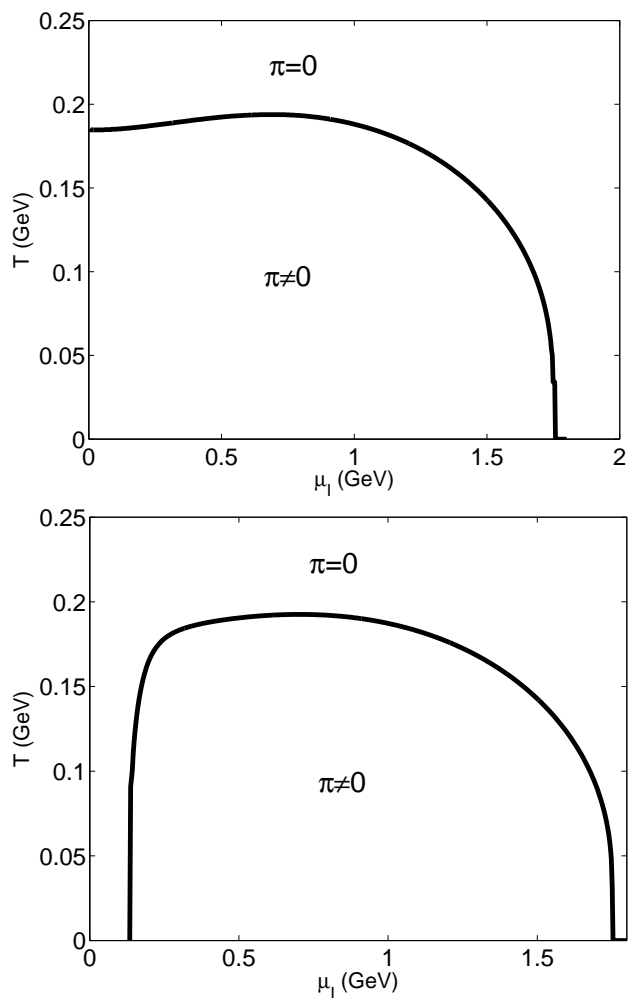

FIG. 12. The phase diagram in the $T-\mu_{I}$ plane in the chiral limit (upper panel) and real world (lower panel) at $\mu_{B}=0$. 


\section{MESONS AT FINITE ISOSPIN DENSITY}

We now investigate meson properties at finite isospin chemical potential. In the NJL model, the meson modes are regarded as quantum fluctuations above the mean field. The meson modes can be calculated in the frame of RPA [27]. When the mean field quark propagator is diagonal, for instance the case with only chiral condensation, the summation of bubbles in RPA selects its specific channel by choosing at each stage the same proper polarization function, a meson mode which is determined by the pole of the corresponding meson propagator is related to its own polarization function $\Pi_{M M}(k)[30,31]$ only,

$$
1-2 G \Pi_{M M}(k)=0 .
$$

However, for the quark propagator with off-diagonal elements, like the cases of $\eta$ and $\eta^{\prime}$ meson spectrum [27], pion superfluidity considered here, and color superconductivity in Section VII, we must consider all possible channels in the bubble summation in RPA. Using matrix notation, the effective quark propagator (14) leads to the meson modes determined by

$$
\operatorname{det}(1-2 G \Pi(k))=0,
$$

with the polarization function matrix

$$
1-2 G \Pi=
$$

$\left(\begin{array}{cccc}1-2 G \Pi_{\sigma \sigma} & -2 G \Pi_{\sigma \pi_{+}} & -2 G \Pi_{\sigma \pi_{-}} & -2 G \Pi_{\sigma \pi_{0}} \\ -2 G \Pi_{\pi_{+} \sigma} & 1-2 G \Pi_{\pi_{+} \pi_{+}} & -2 G \Pi_{\pi_{+} \pi_{-}} & -2 G \Pi_{\pi_{+} \pi_{0}} \\ -2 G \Pi_{\pi_{-} \sigma} & -2 G \Pi_{\pi_{-} \pi_{+}} & 1-2 G \Pi_{\pi_{-} \pi_{-}} & -2 G \Pi_{\pi_{-} \pi_{0}} \\ -2 G \Pi_{\pi_{0} \sigma} & -2 G \Pi_{\pi_{0} \pi_{+}} & -2 G \Pi_{\pi_{0} \pi_{-}} & 1-2 G \Pi_{\pi_{0} \pi_{0}}\end{array}\right)$

Here the polarization functions defined as

$$
\Pi_{M M^{\prime}}(k)=i \int \frac{d^{4} p}{(2 \pi)^{4}} \operatorname{Tr}\left(\Gamma_{M}^{*} \mathcal{S}_{m f}(p+k) \Gamma_{M^{\prime}} \mathcal{S}_{m f}(p)\right)
$$

with the vertexes

$$
\begin{gathered}
\Gamma_{M}= \begin{cases}1 & M=\sigma \\
i \tau_{+} \gamma_{5} & M=\pi_{+} \\
i \tau_{-} \gamma_{5} & M=\pi_{-} \\
i \tau_{3} \gamma_{5} & M=\pi_{0}\end{cases} \\
\Gamma_{M}^{*}= \begin{cases}1 & M=\sigma \\
i \tau_{-} \gamma_{5} & M=\pi_{+} \\
i \tau_{+} \gamma_{5} & M=\pi_{-} \\
i \tau_{3} \gamma_{5} & M=\pi_{0}\end{cases}
\end{gathered}
$$

are shown in Appendix B. Here the trace Tr is taken in color, flavor and Dirac spaces.

As will be seen in the following, in the normal phase with vanished pion condensate, the polarization matrix is diagonal at finite isospin chemical potential and one can then directly use the method developed in $[30,31]$ to calculate the masses of $\sigma, \pi_{+}, \pi_{-}$and $\pi_{0}$. In the superfluidity phase with nonzero $\pi$ and off diagonal matrix elements in the mean field quark propagator, the collective excitations of the system will in principle not be the original meson modes in the vacuum but their mixture. Especially it is expected that there will be a Goldstone mode in the superfluidity phase corresponding to the spontaneously isospin symmetry breaking.

In the following we will study the meson properties in the normal phase $(\pi=0)$ and superfluidity $\operatorname{phase}(\pi \neq 0)$ separately.

\section{A. Meson Properties in Normal Phase}

In the vacuum, due to isospin symmetry, one can regard $\pi_{1}, \pi_{2}$ or $\pi_{+}, \pi_{-}$or any other linear combination of $\pi_{1}, \pi_{2}$ as eigen collective modes. In the normal phase with vanished pion condensate, the polarization function matrix is diagonal with respect to the basis $\left(\sigma, \pi_{+}, \pi_{-}, \pi_{3}\right)$ but not diagonal with respect to the basis $\left(\sigma, \pi_{1}, \pi_{2}, \pi_{3}\right)$. This means that in the normal phase, the eigen collective modes are $\sigma, \pi_{+}, \pi_{-}, \pi_{0}$. Thus the dispersion relation for each mode is determined through the equation

$$
1-2 G \Pi_{M M}(k)=0 .
$$

(51)The meson mass is defined as the root $k_{0}$ at $\mathbf{k}=\mathbf{0}$.

Doing the trace in flavor and color spaces, we obtain the polarization functions in terms of the matrix elements of the quark propagator,

$$
\begin{aligned}
\Pi_{\sigma \sigma}(k) & =i N_{c} \int \frac{d^{4} p}{(2 \pi)^{4}} \operatorname{Tr}_{D}\left(\mathcal{S}_{u u}(p+k) \mathcal{S}_{u u}(p)\right. \\
& \left.+\mathcal{S}_{d d}(p+k) \mathcal{S}_{d d}(p)\right) \\
\Pi_{\pi_{0} \pi_{0}}(k) & =-i N_{c} \int \frac{d^{4} p}{(2 \pi)^{4}} \operatorname{Tr}_{D}\left(\gamma_{5} \mathcal{S}_{u u}(p+k) \gamma_{5} \mathcal{S}_{u u}(p)\right. \\
& \left.+\gamma_{5} \mathcal{S}_{d d}(p+k) \gamma_{5} \mathcal{S}_{d d}(p)\right)
\end{aligned}
$$

$\Pi_{\pi_{+} \pi_{+}}(k)=-2 i N_{c} \int \frac{d^{4} p}{(2 \pi)^{4}} \operatorname{Tr}_{D}\left(\gamma_{5} \mathcal{S}_{u u}(p+k) \gamma_{5} \mathcal{S}_{d d}(p)\right)$,

$\Pi_{\pi_{-} \pi_{-}}(k)=-2 i N_{c} \int \frac{d^{4} p}{(2 \pi)^{4}} \operatorname{Tr}_{D}\left(\gamma_{5} \mathcal{S}_{d d}(p+k) \gamma_{5} \mathcal{S}_{u u}(p)\right)$,

now the trace $\operatorname{Tr}_{D}$ is taken only in Dirac space. At zero temperature and zero baryon density, the polarization functions at $\mathbf{k}=\mathbf{0}$ are simplified as

$$
\begin{aligned}
& \Pi_{\sigma \sigma}\left(k_{0}\right)=4 N_{c} \int \frac{d^{3} \mathbf{p}}{\left(2 \pi^{3}\right)} \frac{1}{E_{p}} \frac{E_{p}^{2}-M_{q}^{2}}{E_{p}^{2}-k_{0}^{2} / 4}, \\
& \Pi_{\pi_{0} \pi_{0}}\left(k_{0}\right)=4 N_{c} \int \frac{d^{3} \mathbf{p}}{\left(2 \pi^{3}\right)} \frac{1}{E_{p}} \frac{E_{p}^{2}}{E_{p}^{2}-k_{0}^{2} / 4}, \\
& \Pi_{\pi_{+} \pi_{+}}\left(k_{0}\right)=4 N_{c} \int \frac{d^{3} \mathbf{p}}{\left(2 \pi^{3}\right)} \frac{1}{E_{p}} \frac{E_{p}^{2}}{E_{p}^{2}-\left(k_{0}+\mu_{I}\right)^{2} / 4},
\end{aligned}
$$




$$
\Pi_{\pi_{-} \pi_{-}}\left(k_{0}\right)=4 N_{c} \int \frac{d^{3} \mathbf{p}}{\left(2 \pi^{3}\right)} \frac{1}{E_{p}} \frac{E_{p}^{2}}{E_{p}^{2}-\left(k_{0}-\mu_{I}\right)^{2} / 4} .
$$

From the comparison with their expressions in the vacuum with $\mu_{I}=0$, we obtain the $\mu_{I}$ dependence of the meson masses,

$$
\begin{aligned}
M_{\sigma}\left(\mu_{I}\right) & =m_{\sigma}, \\
M_{\pi_{0}}\left(\mu_{I}\right) & =m_{\pi}, \\
M_{\pi_{+}}\left(\mu_{I}\right) & =m_{\pi}-\mu_{I}, \\
M_{\pi_{-}}\left(\mu_{I}\right) & =m_{\pi}+\mu_{I} .
\end{aligned}
$$

The mesons which carry zero isospin charge keep their vacuum masses, while the mass of the meson which carries positive (negative) isospin charge decreases (increases) with $\mu_{I}$ linearly. These relations hold before $\mu_{I}=m_{\pi}$ where the pion condensation starts.

After performing Matsubara frequency summation the meson polarization functions at $\mathbf{k}=0$ are explicitly expressed as

$$
\begin{aligned}
& \Pi_{\sigma \sigma}\left(k_{0}\right)=-2 N_{c} \int \frac{d^{3} \mathbf{p}}{\left(2 \pi^{3}\right)} \frac{1}{E_{p}} \frac{E_{p}^{2}-M_{q}^{2}}{E_{p}^{2}-k_{0}^{2} / 4} \\
& \quad \times\left(f\left(E_{-}^{-}\right)+f\left(E_{-}^{+}\right)-f\left(-E_{+}^{-}\right)-f\left(-E_{+}^{+}\right)\right), \\
& \Pi_{\pi_{0} \pi_{0}}\left(k_{0}\right)=-2 N_{c} \int \frac{d^{3} \mathbf{p}}{\left(2 \pi^{3}\right)} \frac{1}{E_{p}} \frac{E_{p}^{2}}{E_{p}^{2}-k_{0}^{2} / 4} \\
& \quad \times\left(f\left(E_{-}^{-}\right)+f\left(E_{-}^{+}\right)-f\left(-E_{+}^{-}\right)-f\left(-E_{+}^{+}\right)\right), \\
& \Pi_{\pi_{+} \pi_{+}}\left(k_{0}\right)=4 N_{c} \int \frac{d^{3} \mathbf{p}}{\left(2 \pi^{3}\right)} \\
& \quad \times\left[\frac{2 E_{p}-\mu_{I}+k_{0}}{k_{0}^{2}-4\left(E_{p}-\mu_{I} / 2\right)^{2}}\left(f\left(E_{-}^{-}\right)-f\left(-E_{+}^{-}\right)\right)\right. \\
& \left.\quad+\frac{2 E_{p}+\mu_{I}-k_{0}}{k_{0}^{2}-4\left(E_{p}+\mu_{I} / 2\right)^{2}}\left(f\left(E_{-}^{+}\right)-f\left(-E_{+}^{+}\right)\right)\right], \\
& \Pi_{\pi_{-} \pi_{-}}\left(k_{0}\right)=4 N_{c} \int \frac{d^{3} \mathbf{p}}{\left(2 \pi^{3}\right)} \\
& \quad \times\left[\frac{2 E_{p}-\mu_{I}-k_{0}}{k_{0}^{2}-4\left(E_{p}-\mu_{I} / 2\right)^{2}}\left(f\left(E_{-}^{-}\right)-f\left(-E_{+}^{-}\right)\right)\right. \\
& \left.\quad+\frac{2 E_{p}+\mu_{I}+k_{0}}{k_{0}^{2}-4\left(E_{p}+\mu_{I} / 2\right)^{2}}\left(f\left(E_{-}^{+}\right)-f\left(-E_{+}^{+}\right)\right)\right] .
\end{aligned}
$$

From the comparison of $\Pi_{\pi_{+} \pi_{+}}$at $\mu_{B}=0$ and $k_{0}=0$ with the last gap equation of (19) for the pion condensate $\pi$, the phase transition line from $\pi=0$ to $\pi \neq 0$ in the $T-\mu_{I}$ plane at $\mu_{B}=0$ can be calculated by the equation

$$
1-2 G \Pi_{\pi_{+} \pi_{+}}\left(k_{0}=0\right)=0 .
$$

Thus the $\pi_{+}$mass is always zero on the phase boundary.

\section{B. Meson Properties in Superfluidity Phase}

The polarization functions $\Pi_{M M^{\prime}}\left(k_{0}\right)$ at $\mathbf{k}=0$ are calculated in Appendix B as explicit functions of temperature $T$, baryon chemical potential $\mu_{B}$, and isospin chemical potential $\mu_{I}$ in general case with nonzero chiral and pion condensates. While $\sigma, \pi_{+}$and $\pi_{-}$are no longer collective excitation modes due to the pion condensation, there is still no mixing between $\pi_{0}$ and other mesons,

$$
\Pi_{\pi_{0} \sigma}(k)=\Pi_{\pi_{0} \pi_{+}}(k)=\Pi_{\pi_{0} \pi_{-}}(k)=0,
$$

its mass in the superfluidity phase is determined by

$$
1-2 G \Pi_{\pi_{0} \pi_{0}}\left(k_{0}=M_{\pi_{0}}, \mathbf{k}=0\right)=0 .
$$

Taking the polarization function $\Pi_{\pi_{0} \pi_{0}}$ evaluated in Appendix $\mathrm{B}$ and making comparison with the gap equation for $\pi$ at $T=\mu_{B}=0$, we get analytically

$$
M_{\pi_{0}}\left(\mu_{I}\right)=\mu_{I}, \quad \mu_{I}>m_{\pi} .
$$

The other meson masses are now determined by the equation

$$
\begin{aligned}
& \operatorname{det}\left(\begin{array}{ccc}
1-2 G \Pi_{\sigma \sigma} & -2 G \Pi_{\sigma \pi_{+}} & -2 G \Pi_{\sigma \pi_{-}} \\
-2 G \Pi_{\pi_{+} \sigma} & 1-2 G \Pi_{\pi_{+} \pi_{+}} & -2 G \Pi_{\pi_{+} \pi_{-}} \\
-2 G \Pi_{\pi_{-} \sigma} & -2 G \Pi_{\pi_{-} \pi_{+}} & 1-2 G \Pi_{\pi_{-} \pi_{-}}
\end{array}\right) \\
& =0
\end{aligned}
$$

at $\mathbf{k}=0$, namely,

$$
\begin{aligned}
& {\left[\left(\left(1-2 G \Pi_{\pi_{+} \pi_{+}}\left(k_{0}\right)\right)\left(1-2 G \Pi_{\pi_{-} \pi_{-}}\left(k_{0}\right)\right)-4 G^{2} \Pi_{\pi_{+} \pi_{-}}^{2}\left(k_{0}\right)\right)\right.} \\
& \times\left(1-2 G \Pi_{\sigma \sigma}\left(k_{0}\right)\right)-16 G^{3} \Pi_{\sigma \pi_{+}}\left(k_{0}\right) \Pi_{\sigma \pi_{-}}\left(k_{0}\right) \Pi_{\pi_{+} \pi_{-}}\left(k_{0}\right) \\
& -4 G^{2} \Pi_{\sigma \pi_{-}}^{2}\left(k_{0}\right)\left(1-2 G \Pi_{\pi_{+} \pi_{+}}\left(k_{0}\right)\right)-4 G^{2} \Pi_{\sigma \pi_{+}}^{2}\left(k_{0}\right) \\
& \left.\times\left(1-2 G \Pi_{\pi_{-} \pi_{-}}\left(k_{0}\right)\right)\right]_{k_{0}=M}=0 .
\end{aligned}
$$

We first discuss the masses in the chiral limit. In the chiral limit, the chiral condensate $\sigma$ as well as the effective quark mass $M_{q}$ are forced to be zero by any small isospin chemical potential. From the explicit form of the polarization functions evaluated in Appendix B, the mixing between $\sigma$ and $\pi_{ \pm}$disappears, $\Pi_{\sigma \pi_{ \pm}}=0$, then $\sigma$ is still an eigen collective mode. Since the polarization functions for $\sigma$ and $\pi_{0}$ are exactly the same in this case, $\Pi_{\sigma \sigma}=\Pi_{\pi_{0} \pi_{0}}$, the mesons $\pi^{0}$ and $\sigma$ have the same mass in the superfluidity phase,

$$
M_{\sigma}\left(T, \mu_{I}, \mu_{B}\right)=M_{\pi_{0}}\left(T, \mu_{I}, \mu_{B}\right),
$$

for any $T, \mu_{I}$ and $\mu_{B}$, which reflects the restoration of chiral symmetry. In the case of $T=\mu_{B}=0$ we have

$$
M_{\sigma}\left(\mu_{I}\right)=M_{\pi_{0}}\left(\mu_{I}\right)=\mu_{I} .
$$

The other two eigen modes are determined by 


$$
\begin{aligned}
& {\left[\left(1-2 G \Pi_{\pi_{+} \pi_{+}}\left(k_{0}\right)\right)\left(1-2 G \Pi_{\pi_{-} \pi_{-}}\left(k_{0}\right)\right)\right.} \\
& \left.\quad-4 G^{2} \Pi_{\pi_{+} \pi_{-}}^{2}\left(k_{0}\right)\right]_{k_{0}=M}=0 .
\end{aligned}
$$

From the polarization functions shown in Appendix B, it is easy to prove that $M=0$ is a solution of (67) corresponding to the spontaneously isospin symmetry breaking. We call the massless meson mode as $\pi_{L}$ and the other heavy mode as $\pi_{H}$. The meson mass spectra in the chiral limit are shown in Fig.(13) as functions of $\mu_{I}$ at $T=\mu_{B}=0$.

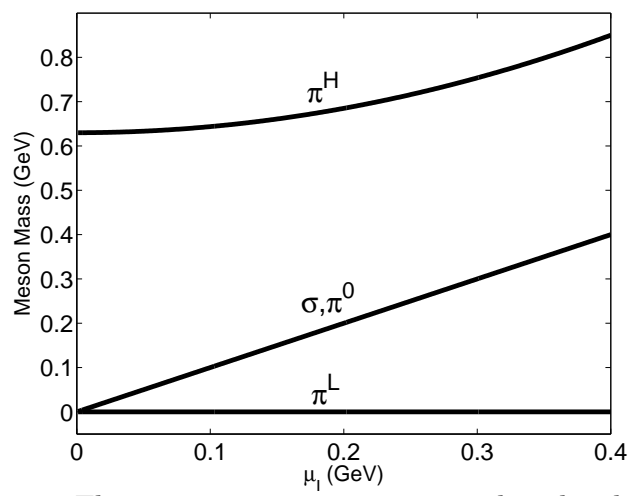

FIG. 13. The meson mass spectra in the chiral limit as functions of $\mu_{I}$ at $T=\mu_{B}=0$.

In the real world with nonzero current quark mass, there is not only the mixing between the charged pions, but also a mixing between $\sigma$ and the charged pions. From the explicit expression of the polarization functions shown in Appendix B, we have

$$
\begin{aligned}
& \Pi_{\sigma \pi_{ \pm}}=\Pi_{\pi_{ \pm} \sigma} \propto \sqrt{2} M_{q}|2 G \pi|, \\
& \Pi_{\pi_{+} \pi_{-}}=\Pi_{\pi_{-} \pi_{+}} \propto 4 G^{2} \pi^{2} .
\end{aligned}
$$

To quantitatively see the mixing degree among $\sigma, \pi_{+}$and $\pi_{-}$, we introduce two dimensionless parameters,

$$
\begin{aligned}
& \eta_{\sigma \pi}=\frac{\sqrt{2} M_{q}|2 G \pi|}{4 G^{2} \sigma_{0}^{2}} \\
& \eta_{\pi \pi}=\frac{4 G^{2} \pi^{2}}{4 G^{2} \sigma_{0}^{2}}
\end{aligned}
$$

describing the strengthes of $\sigma-\pi$ mixing and $\pi-\pi$ mixing. Their isospin dependence is shown in Fig.(14) at $T=\mu_{B}=0$. The $\sigma-\pi$ mixing is much weaker than the $\pi-\pi$ mixing, since the former is proportional to both chiral condensate and pion condensate, but the latter to the square of pion condensate, and in the superfluidity phase the chiral symmetry is almost restored. The $\sigma-\pi$ mixing is important only around the phase transition point $\mu_{I}^{c}=m_{\pi}$, but the $\pi-\pi$ mixing is strong in a wide region after the phase transition.

The mass spectra calculated through solving the Eq.(64) numerically are shown in Fig.(15), together with $M_{\pi_{0}}$, Eq.(62) in the superfluidity phase and the masses
(57) in the normal phase, as functions of $\mu_{I}$ at $T=\mu_{B}=$ 0 . Again we obtain the Goldstone mode reflecting correctly the spontaneous isospin symmetry breaking in the superfluidity phase. In the normal phase $\sigma, \pi_{+}, \pi_{-}$and $\pi_{0}$ themselves are eigen modes of the collective excitation of the system, but in the superfluidity phase, except for $\pi_{0}$ which is still an eigen mode, $\sigma, \pi_{+}$and $\pi_{-}$are no longer eigen modes. Since the masses determined by (64) are continuously connected with the masses of $\sigma, \pi_{+}$ and $\pi_{-}$at the phase transition point $\mu_{I}=m_{\pi}$, we call them $\Sigma, \Pi_{+}$and $\Pi_{-}$, shown in Fig.(15). When $\mu_{I}$ is high enough, the pion condensation will disappear due to the asymptotic freedom of QCD, this turning point in the NJL model is about $\tilde{\mu}_{I}^{c}=1.75 \mathrm{GeV}$, see Fig.(1). Above this turning point the mass spectra are again controlled by the polarization functions (56) and the $\mu_{I}$ dependence is similar to $(57)$.

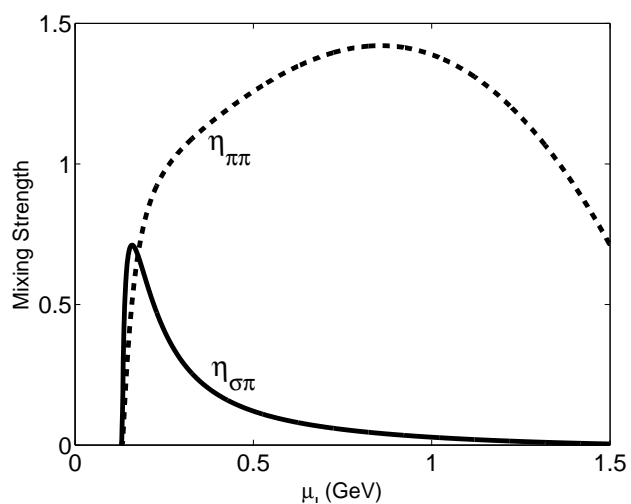

FIG. 14. The mixing strengthes $\eta_{\sigma \pi}$ and $\eta_{\pi \pi}$ as functions of isospin chemical potential $\mu_{I}$ at $T=\mu_{B}=0$.

The mass spectra in the superfluidity phase calculated in the NJL model here are very similar to the result predicted in chiral perturbation theory $[8,12]$,

$$
\begin{aligned}
& M_{\Pi_{+}}=0 \\
& M_{\Pi_{-}}=\mu_{I} \sqrt{1+3 m_{\pi}^{4} / \mu_{I}^{4}}, \\
& M_{\pi_{0}}=\mu_{I}
\end{aligned}
$$

However, if we neglect the $\sigma-\pi$ mixing in the superfluidity phase, $\sigma$ is still the eigen mode and the mass is calculated from the pole equation

$$
1-2 G \Pi_{\sigma \sigma}\left(k_{0}=M_{\sigma}, \mathbf{k}=0\right)=0 \text {, }
$$

and the other two eigen modes which are linear combinations of $\pi_{+}$and $\pi_{-}$are determined by the equation (67). One of the modes is still the Goldstone mode and the other is a heavy one. The numerical results for $\sigma$ and the heavy one are also shown in Fig.(15) as dashed lines. In this case, the broken $U_{A}(1)$ symmetry will be restored at sufficiently high isospin density, indicating by the same $\sigma$ and $\pi_{0}$ mass. It is clear that the difference between the full calculation and the approximation neglecting $\sigma-\pi$ 
mixing is mainly in the narrow region above the phase transition point $\mu_{I}=m_{\pi}$.

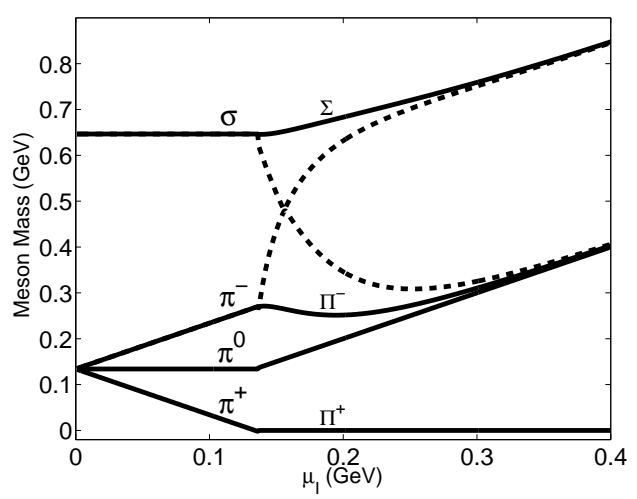

FIG. 15. The meson masses as functions of isospin chemical potential $\mu_{I}$ at $T=\mu_{B}=0$ in the real world. The solid lines are the full calculation and the dashed lines from the approximation neglecting $\sigma-\pi$ mixing.

We now analytically prove the Goldstone mode in general case in the NJL model. To this end we consider the polarization function matrix

$$
\begin{aligned}
& 1-2 G \Pi= \\
& \left(\begin{array}{cccc}
1-2 G \Pi_{00} & -2 G \Pi_{01} & -2 G \Pi_{02} & -2 G \Pi_{03} \\
-2 G \Pi_{10} & 1-2 G \Pi_{11} & -2 G \Pi_{12} & -2 G \Pi_{13} \\
-2 G \Pi_{20} & -2 G \Pi_{21} & 1-2 G \Pi_{22} & -2 G \Pi_{23} \\
-2 G \Pi_{30} & -2 G \Pi_{31} & -2 G \Pi_{32} & 1-2 G \Pi_{33}
\end{array}\right)
\end{aligned}
$$

with respect to the basis $\left(\sigma, \pi_{1}, \pi_{2}, \pi_{3}\right)$.

From Appendix B, $\pi_{3}$ does not mix with other modes,

$$
\Pi_{3 n}(k)=\Pi_{n 3}(k)=0, \quad n=0,1,2,
$$

its mass is determined by its own polarization function at $\mathbf{k}=0$,

$$
1-2 G \Pi_{33}\left(k_{0}=M_{\pi_{0}}\right)=0
$$

The masses of the other modes are solved from the equation

$$
\begin{aligned}
& \operatorname{det}\left(\begin{array}{ccc}
1-2 G \Pi_{00} & -2 G \Pi_{01} & -2 G \Pi_{02} \\
-2 G \Pi_{10} & 1-2 G \Pi_{11} & -2 G \Pi_{12} \\
-2 G \Pi_{20} & -2 G \Pi_{21} & 1-2 G \Pi_{22}
\end{array}\right)_{k_{0}=M} \\
& =0
\end{aligned}
$$

at $\mathbf{k}=0$. The existence of the Goldstone mode means that $M=0$ is a solution of the above equation. This is really true. In fact, setting $k_{0}=\mathbf{k}=0$ we have from the Appendix B

$$
\Pi_{02}(0, \mathbf{0})=\Pi_{20}(0, \mathbf{0})=\Pi_{12}(0, \mathbf{0})=\Pi_{21}(0, \mathbf{0})=0,
$$

the Goldstone mode can be checked by the equation

$$
1-2 G \Pi_{22}\left(k_{0}=0, \mathbf{k}=0\right)=0 \text {. }
$$

Using the analytical form of $\Pi_{22}$ evaluated in Appendix $\mathrm{B}$, we find that the above equation is exactly the same as the gap equation for the pion condensate. Therefore, in superfluidity phase with $\pi \neq 0$, there is always a Goldstone mode. Note that this massless mode holds in the whole superfluidity phase at finite temperature and isospin and baryon chemical potentials.

The Goldstone mode will extremely change the thermodynamics of the system. In mean field approximation, only quarks contribute to the thermodynamic potential, see (26). Since quarks are heavy at low temperature, the thermodynamic functions scaled by the temperature, for example, $p / T^{4}, \epsilon / T^{4}, s / T^{3}$ and $c / T^{3}$ approach to zero in the limit of $T \rightarrow 0$ [31]. However, when the meson fluctuations are included, the massless meson mode will lead to power laws of thermodynamic functions at low temperature, these scaled functions will approach to nonzero constants.

\section{PION SUPERFLUIDITY IN LINEAR SIGMA MODEL}

In this section we study the linear sigma model at finite isospin chemical potential and compare it with the NJL model. Since the linear sigma model can be regarded as a bosonized version of the NJL model, we want to know whether the former can reproduce the results we have worked out with the latter with quarks as elementary degrees of freedom. It is well-known that one can use the bosonization technique to integrate out the quark degrees of freedom and obtain the effective Lagrangian for the meson bound states in the NJL model $[27,43]$. In the real world the Lagrangian is just the one of the linear sigma model with an explicit chiral breaking term,

$$
\begin{aligned}
\mathcal{L} & =\frac{1}{2}\left[\left(\partial_{\mu} \sigma\right)^{2}+\left(\partial_{\mu} \vec{\pi}\right)^{2}\right]+\frac{1}{2}\left(2 g_{\pi}^{2} f_{\pi}^{2}-m_{\pi}^{2}\right)\left(\sigma^{2}+\vec{\pi}^{2}\right) \\
& -\frac{g_{\pi}^{2}}{2}\left(\sigma^{2}+\vec{\pi}^{2}\right)^{4}-f_{\pi} m_{\pi}^{2} \sigma
\end{aligned}
$$

where $g_{\pi}=g_{\pi q \bar{q}}$ is the pion-quark-antiquark coupling constant, $f_{\pi}$ the pion decay constant, and $m_{\pi}$ the pion mass in the vacuum, $g_{\pi}$ and $f_{\pi}$ can be calculated in the NJL model in RPA.

\section{A. Zero Temperature}

Introducing the isospin chemical potential $\mu_{I}$ corresponding to the third component of isospin charge

$$
I_{3}=\int d^{3} \mathbf{x}\left(\pi_{1} \partial_{t} \pi_{2}-\pi_{2} \partial_{t} \pi_{1}\right),
$$

and the chiral and pion condensates 


$$
\begin{aligned}
& \langle\sigma\rangle=\xi, \\
& \left\langle\pi^{+}\right\rangle=\left\langle\pi^{-}\right\rangle=\frac{\rho}{\sqrt{2}},
\end{aligned}
$$

we obtain the effective Lagrangian at tree level,

$$
\begin{aligned}
\mathcal{L}_{e f f} & =-U(\xi, \rho)+\frac{1}{2}\left[\left(\partial_{\mu} \sigma\right)^{2}+\left(\partial_{\mu} \pi_{3}\right)^{2}+\left(\partial_{t} \pi_{1}-\mu_{I} \pi_{2}\right)^{2}\right. \\
& \left.+\left(\partial_{t} \pi_{2}+\mu_{I} \pi_{1}\right)^{2}-\left(\nabla \pi_{1}\right)^{2}-\left(\nabla \pi_{2}\right)^{2}\right] \\
& -g_{\pi}^{2}\left[\left(3 \xi^{2}+\rho^{2}-f_{\pi}^{2}+\frac{m_{\pi}^{2}}{2 g_{\pi}^{2}}\right) \sigma^{2}\right. \\
& +\left(\xi^{2}+3 \rho^{2}-f_{\pi}^{2}+\frac{m_{\pi}^{2}}{2 g_{\pi}^{2}}\right) \pi_{1}^{2} \\
& \left.+\left(\xi^{2}+\rho^{2}-f_{\pi}^{2}+\frac{m_{\pi}^{2}}{2 g_{\pi}^{2}}\right)\left(\pi_{2}^{2}+\pi_{3}^{2}\right)+4 \xi \rho \sigma \pi_{1}\right] \\
& -2 g_{\pi}^{2}\left(\xi \sigma+\rho \pi_{1}\right)\left(\sigma^{2}+\pi^{2}\right)-\frac{g_{\pi}^{2}}{2}\left(\sigma^{2}+\pi^{2}\right)^{2}, \quad(81)
\end{aligned}
$$

with the classical potential

$$
\begin{aligned}
U(\xi, \rho) & =-\frac{1}{2}\left(2 g_{\pi}^{2} f_{\pi}^{2}-m_{\pi}^{2}\right)\left(\xi^{2}+\rho^{2}\right)+\frac{g_{\pi}^{2}}{2}\left(\xi^{2}+\rho^{2}\right)^{2} \\
& -f_{\pi} m_{\pi}^{2} \xi-\frac{1}{2} \mu_{I}^{2} \rho^{2}
\end{aligned}
$$

where the two condensates are determined by minimizing the potential,

$$
\frac{\partial U}{\partial \xi}=0, \quad \frac{\partial U}{\partial \rho}=0 .
$$

We have chosen pion condensate to be real as in the NJL model. In this section we use $\xi$ and $\rho$ standing for the chiral and pion condensates in order to avoid confusion with the definitions in the NJL model.

In the vacuum the constraints (83) on the potential give $\xi=f_{\pi}$ and $\rho=0$, and the sigma mass can be read out from the quadratic term in the sigma field in the effective Lagrangian (81), $m_{\sigma}^{2}=4 g_{\pi}^{2} f_{\pi}^{2}+m_{\pi}^{2}$.

At finite isospin density the two condensates in the ground state satisfy $\xi=0, \rho=f_{\pi} \sqrt{1+2 \mu_{I}^{2} / m_{\sigma}^{2}}$ in the chiral limit. This is consistent with the NJL model: Any small isospin density can force the chiral symmetry to be restored in the chiral limit. In the real world, we obtain $\xi=f_{\pi}, \rho=0$ for $\mu_{I}<m_{\pi}$ which is the same as in the vacuum, and [5]

$$
\begin{aligned}
& \xi=f_{\pi} \frac{m_{\pi}^{2}}{\mu_{I}^{2}} \\
& \rho=f_{\pi} \sqrt{1-\left(\frac{m_{\pi}^{2}}{\mu_{I}^{2}}\right)^{2}+2 \frac{\mu_{I}^{2}-m_{\pi}^{2}}{m_{\sigma}^{2}-m_{\pi}^{2}}},
\end{aligned}
$$

for $\mu_{I}>m_{\pi}$. This result is qualitatively consistent with the NJL model and the chiral perturbation theory. Especially the critical behaviors of the two condensates around the phase transition point $\mu_{I}^{c}=m_{\pi}$ in the three effective models are almost the same.

The thermodynamic functions can be evaluated from the potential $U$. The isospin density $n_{I}$, pressure $p$ and energy density $\epsilon$ are analytically written as

$$
\begin{aligned}
n_{I} & =-\frac{\partial U}{\partial \mu_{I}}=\mu_{I} \rho^{2} \\
& =\mu_{I} f_{\pi}^{2}\left(1-\frac{m_{\pi}^{4}}{\mu_{I}^{4}}+2 \frac{\mu_{I}^{2}-m_{\pi}^{2}}{m_{\sigma}^{2}-m_{\pi}^{2}}\right), \\
p & =-U(\xi, \rho)+U\left(f_{\pi}, 0\right) \\
& =\frac{1}{2} f_{\pi}^{2}\left(\mu_{I}^{2}-m_{\pi}^{2}\right)^{2}\left(\frac{1}{\mu_{I}^{2}}+\frac{1}{m_{\sigma}^{2}-m_{\pi}^{2}}\right), \\
\epsilon & =-p+\mu_{I} n_{I} \\
& =\frac{1}{2} f_{\pi}^{2}\left(\mu_{I}^{2}-m_{\pi}^{2}\right)\left(\frac{\mu_{I}^{2}+3 m_{\pi}^{3}}{\mu_{I}^{2}}+\frac{3 \mu_{I}^{2}+m_{\pi}^{2}}{m_{\sigma}^{2}-m_{\pi}^{2}}\right) .
\end{aligned}
$$
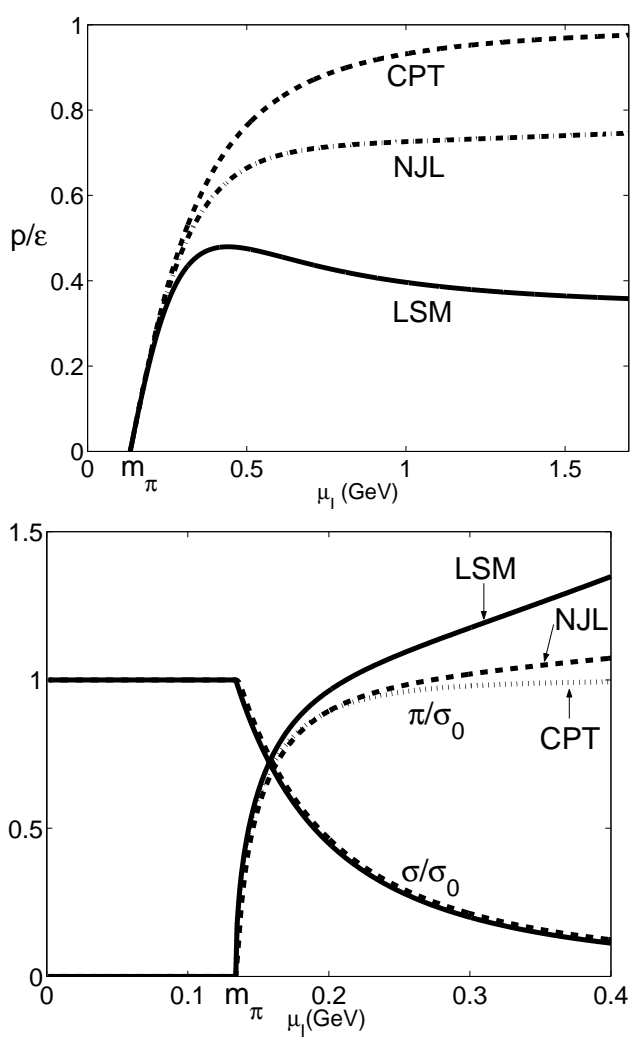

FIG. 16. The ratio $p / \epsilon$ (upper panel) and the chiral and pion condensates (lower panel) as functions of isospin chemical potential $\mu_{I}$ at $T=\mu_{B}=0$ in the NJL model (NJL), linear sigma model (LSM), and chiral perturbation theory (CPT).

If we take the limit $m_{\sigma} \rightarrow \infty$, we reproduce the result in the chiral perturbation theory $[8,12]$. However, the finite sigma mass leads to a big difference at large $\mu_{I}$. At large $\mu_{I}$, the ratio $p / \epsilon$ approaches 1 in the chiral perturbation theory, but only about $1 / 3$ in the linear sigma model and 0.7 in the NJL model, as shown in Fig.(16). While the three effective models are very different in high 
density region, they behavior almost the same in the region close to the critical point, see also the chiral and pion condensates shown in the lower panel of (16).

The meson mass spectrum is easily obtained by diagonalizing the quadratic part of the effective Lagrangian (81). It is clear that $\pi_{0}$ itself is an eigen mode of the system, while $\pi_{+}$and $\pi_{-}$are not, like the case in the NJL model. A simple algebra calculation gives the result

$$
\begin{aligned}
& M_{\sigma}\left(\mu_{I}\right)=m_{\sigma}, \\
& M_{\pi^{-}}\left(\mu_{I}\right)=m_{\pi}+\mu_{I}, \\
& M_{\pi^{+}}\left(\mu_{I}\right)=m_{\pi}-\mu_{I}, \\
& M_{\pi_{0}}\left(\mu_{I}\right)=m_{\pi},
\end{aligned}
$$

for $\mu_{I}<m_{\pi}$ and

$$
\begin{aligned}
& M_{\Sigma}\left(\mu_{I}\right)= \\
& \sqrt{2 g_{\pi}^{2} f_{\pi}^{2}+\frac{7 \mu_{I}^{2}}{2}-m_{\pi}^{2}+2 g_{\pi}^{2} \sqrt{\left(\xi^{2}-\rho^{2}-\frac{3 \mu_{I}^{2}}{4 g_{\pi}^{2}}\right)^{2}+4 \xi^{2} \rho^{2}}}, \\
& M_{\Pi_{-}}\left(\mu_{I}\right)= \\
& \sqrt{2 g_{\pi}^{2} f_{\pi}^{2}+\frac{7 \mu_{I}^{2}}{2}-m_{\pi}^{2}-2 g_{\pi}^{2} \sqrt{\left(\xi^{2}-\rho^{2}-\frac{3 \mu_{I}^{2}}{4 g_{\pi}^{2}}\right)^{2}+4 \xi^{2} \rho^{2}}}, \\
& M_{\Pi_{+}}\left(\mu_{I}\right)=0, \\
& M_{\pi_{0}}\left(\mu_{I}\right)=\mu_{I},
\end{aligned}
$$

for $\mu_{I}>m_{\pi}$. The numerical result is shown in $\operatorname{Fig}(17)$. The $\sigma-\pi$ mixing here plays also an important role for the meson mass spectra. The calculation without considering $\sigma-\pi$ mixing is indicated by dashed lines. We find that the mass spectra are qualitatively consistent with the NJL model and the chiral perturbation theory.

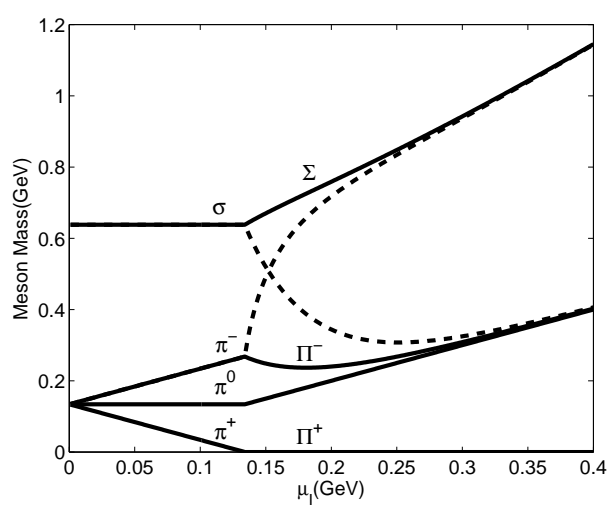

FIG. 17. The meson masses as functions of isospin chemical potential $\mu_{I}$ at $T=0$ in the linear sigma model. The solid lines are the full calculation and the dashed lines from the approximation neglecting $\sigma-\pi$ mixing.

\section{B. Finite Temperature}

There are two approaches to treat thermal excitation in the linear sigma model, the Hartree-Fock approxima- tion and the large $N$ expansion. It is well-known that a disadvantage of the Hartree-Fock approach in the discussion of chiral symmetry restoration is the lack of the Goldstone mode in the symmetry breaking phase [44]. Here we calculate the mass spectra and phase diagram in the two approaches at finite temperature and isospin density and compare the results with the NJL calculation.

Since the sigma model (78) can be regarded as a $O(4)$ model, we adopt the large $N$ expansion method in the $O(N)$ sigma model with boson chemical potential. With the same method and technics given by Harber and Weldon [45], the mass parameter $M$ and condensates $\xi$ and $\rho$ are determined by the following gap equations

$$
\begin{aligned}
& M^{2}=2 g_{\pi}^{2}\left[\xi^{2}+\rho^{2}-f_{\pi}^{2}+2 J^{\prime}\left(T, \mu_{I}, M\right)\right]+m_{\pi}^{2}, \\
& \rho\left(M^{2}-\mu_{I}^{2}\right)=0, \\
& \xi M^{2}-f_{\pi} m_{\pi}^{2}=0,
\end{aligned}
$$

the thermal excitation is introduced by the function

$$
\begin{aligned}
& J^{\prime}\left(T, \mu_{I}, M\right)=\frac{1}{2} \int \frac{d^{3} \mathbf{k}}{(2 \pi)^{3}} \frac{1}{\sqrt{k^{2}+M^{2}}} \\
& \times\left[2 f_{b}(T, 0, M)+f_{b}\left(T, \mu_{I}, M\right)+f_{b}\left(T,-\mu_{I}, M\right)\right],
\end{aligned}
$$

with the Bose-Einstein distribution function

$$
f_{b}\left(T, \mu_{I}, M\right)=\frac{1}{e^{\left(\sqrt{\mathbf{k}^{2}+M^{2}}-\mu_{I}\right) / T}-1} .
$$

It is easy to derive the solution

$$
\begin{aligned}
& M\left(T, \mu_{I}\right)=\mu_{I}, \\
& \xi\left(T, \mu_{I}\right)=f_{\pi} \frac{m_{\pi}^{2}}{\mu_{I}^{2}}, \\
& \rho\left(T, \mu_{I}\right)=\sqrt{\rho^{2}\left(0, \mu_{I}\right)-2 J^{\prime}\left(T, \mu_{I}, \mu_{I}\right)},
\end{aligned}
$$

in the pion superfluidity phase, and the coupled equations

$$
\begin{aligned}
& M^{2}=2 g_{\pi}^{2}\left[\xi^{2}-f_{\pi}^{2}+2 J^{\prime}\left(T, \mu_{I}, M\right)\right]+m_{\pi}^{2}, \\
& \xi=f_{\pi} \frac{m_{\pi}^{2}}{M^{2}},
\end{aligned}
$$

for $M$ and $\xi$ in the normal phase with $\rho=0$. From the above two groups of solutions we can obtain the phase diagram in the $T-\mu_{I}$ plane, shown in Fig.(18).

The Hartree-Fock approach in the superfluidity phase is rather complicated, and there is no Goldstone mode at finite temperature in this approach. Here we will not present the treatment in the superfluidity phase with $\rho \neq$ 0 . However, to calculate the phase transition line of pion superfluidity in the $T-\mu_{I}$ plane is much easier. We need only the formulas in the normal phase with $\rho=0$. From the detailed derivation given in Appendix C, the effective meson masses and the chiral condensate in this phase are governed by the coupled equations, 
$M_{\sigma}^{2}=2 g_{\pi}^{2}\left(3 \xi^{2}-f_{\pi}^{2}+3\langle\sigma \sigma\rangle+2\langle\pi \pi\rangle+\left\langle\pi_{0} \pi_{0}\right\rangle\right)+m_{\pi}^{2}$,

$M_{\pi_{0}}^{2}=2 g_{\pi}^{2}\left(\xi^{2}-f_{\pi}^{2}+\langle\sigma \sigma\rangle+2\langle\pi \pi\rangle+3\left\langle\pi_{0} \pi_{0}\right\rangle\right)+m_{\pi}^{2}$,

$M_{\pi_{ \pm}}^{2}=2 g_{\pi}^{2}\left(\xi^{2}-f_{\pi}^{2}+\langle\sigma \sigma\rangle+4\langle\pi \pi\rangle+\left\langle\pi_{0} \pi_{0}\right\rangle\right)+m_{\pi}^{2}$,

$2 g_{\pi}^{2} \xi\left(\xi^{2}-f_{\pi}^{2}+3\langle\sigma \sigma\rangle+2\langle\pi \pi\rangle+\left\langle\pi_{0} \pi_{0}\right\rangle\right)+\xi m_{\pi}^{2}=f_{\pi} m_{\pi}^{2}$.

To illustrate how to determine the critical temperature from the quantities obtained in the normal phase, we consider the isospin density derived from the thermodynamic potential shown in Appendix C,

$n_{I}=-\frac{\partial \Omega}{\partial \mu_{I}}=\int \frac{d^{3} \mathbf{k}}{\left(2 \pi^{3}\right)}\left(f_{b}\left(T, \mu_{I}, M_{\pi}\right)+f_{b}\left(T,-\mu_{I}, M_{\pi}\right)\right)$.

Note that this is only the thermal contribution to the isospin density and true only in the normal phase. In the superfluidity phase we should include the contribution from the condensation of charged pions. From the definition of Bose-Einstein condensation, the phase transition point where the occupation number density is divergent at zero momentum is determined by

$$
M_{\pi}\left(T_{c}, \mu_{I}\right)=\mu_{I} .
$$

We have checked that this equation can also be derived from the the superfluidity phase in the limit of $\rho=0$.
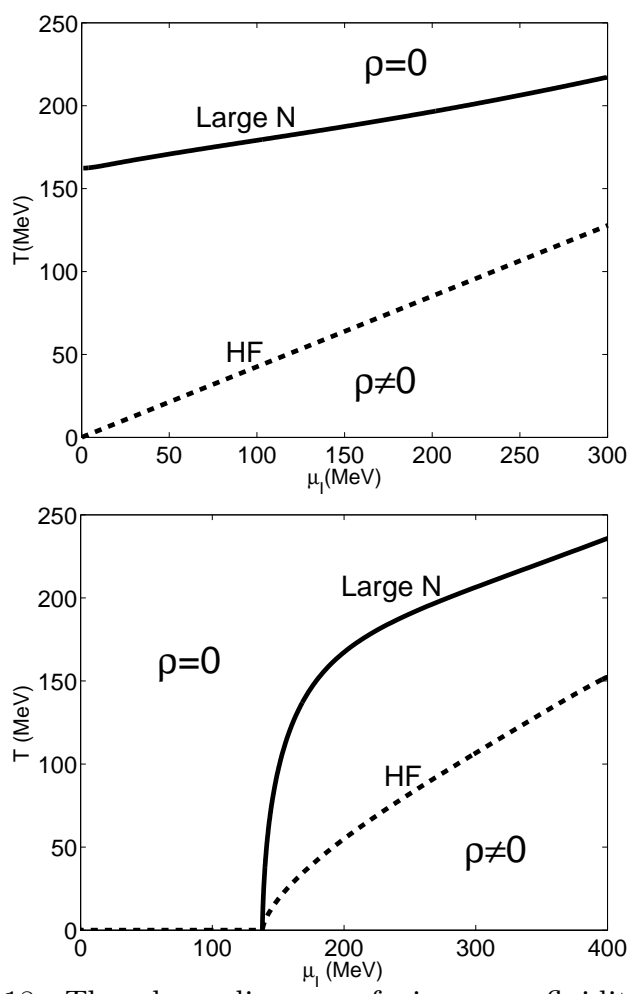

FIG. 18. The phase diagram of pion superfluidity in the $T-\mu_{I}$ plane in the chiral limit (upper panel) and the real world (lower panel) in the linear sigma model. The solid and dashed lines are, respectively, the results of the large $N$ expansion and the Hartree-Fock approximation.
The phase diagram in the $T-\mu_{I}$ plane calculated with the large $N$ expansion method and Hartree-Fock approximation is shown in Fig.(18) in the chiral limit (upper panel) and the real world (lower panel). It is clear that the result of the large $N$ expansion is qualitatively consistent with the NJL model calculation. Especially, in the chiral limit, when $\mu_{I} \rightarrow 0$ the critical temperature in the large $N$ approach is very close to the one obtained in the NJL model, while in the Hartree-Fock approximation the critical temperature approaches zero, which is certainly wrong.

\section{FINITE BARYON DENSITY AND DIQUARK CONDENSATION}

We have investigated the NJL model at finite isospin chemical potential $\mu_{I}$ and temperature $T$ and made comparison with the other effective models. We now turn to the discussion at finite baryon chemical potential $\mu_{B}$. It is well-known that $[7,27,47]$ the baryon density effect on deconfinement and chiral restoration is qualitatively different from the temperature effect: the phase transition is of first order at high baryon density but of second order or even a smooth crossover at high temperature. The physics in high baryon density region is also very different from that in high isospin density region: the spontaneously broken symmetry is of color symmetry at sufficiently high baryon chemical potential but isospin symmetry at sufficiently high isospin chemical potential, the former is described by the diquark condensate $\langle\psi \psi\rangle$ and the latter by the pion condensate $\pi$. We will first consider the baryon density effect on the pion condensation without considering the color superconductivity, and then discuss the competition between the pion superfluidity and color superconductivity in the $\mu_{B}-\mu_{I}$ plane.

\section{A. Finite Baryon Density Effect without Diquark Condensate}

As we indicated in the Section B, when the two chemical potentials $\mu_{B}$ and $\mu_{I}$ are both nonzero, the $u$ and $d$ quark condensates are different from each other, see (19). It is clear that the isospin chemical potential $\mu_{I}$ should be large enough to guarantee the phase transition of pion superfluidity at finite baryon chemical potential $\mu_{B}$ and temperature $T$, at least it should be larger than the critical value $m_{\pi}$ at $T=\mu_{B}=0$. In our numerical calculation we took it as $0.2 \mathrm{GeV}$. The $\mu_{B}$ dependence of the chiral and pion condensates, again scaled by the chiral condensate $\sigma_{0}$ in the vacuum, at fixed $T$ and $\mu_{I}$ is shown in Fig.(19). At zero temperature, the $u$ and $d$ quark condensates $\sigma_{u}$ and $\sigma_{d}$ are almost the same, and all the three condensates, $\sigma_{u}, \sigma_{d}$ and $\pi$ keep their vacuum 
values in the pion superfluidity phase. At a critical value $\mu_{B} \sim 0.85 \mathrm{GeV}$, a first order phase transition happens, the pion condensate jumps down from its vacuum value to zero, and the $u$ and $d$ quark condensates jump up from their vacuum value to some larger values. In the normal phase with $\pi=0$, the two chiral condensates decrease with increasing baryon chemical potential, and the difference between them becomes remarkable. The temperature effect changes the phase transition from first order to second order, see the lower panel for $T=0.1 \mathrm{GeV}$, and reduces the critical baryon chemical potential from about $0.85 \mathrm{GeV}$ to about $0.65 \mathrm{GeV}$. Also the temperature effect leads to remarkable difference between the two chiral condensates not only in the normal phase but also in the pion superfluidity phase.
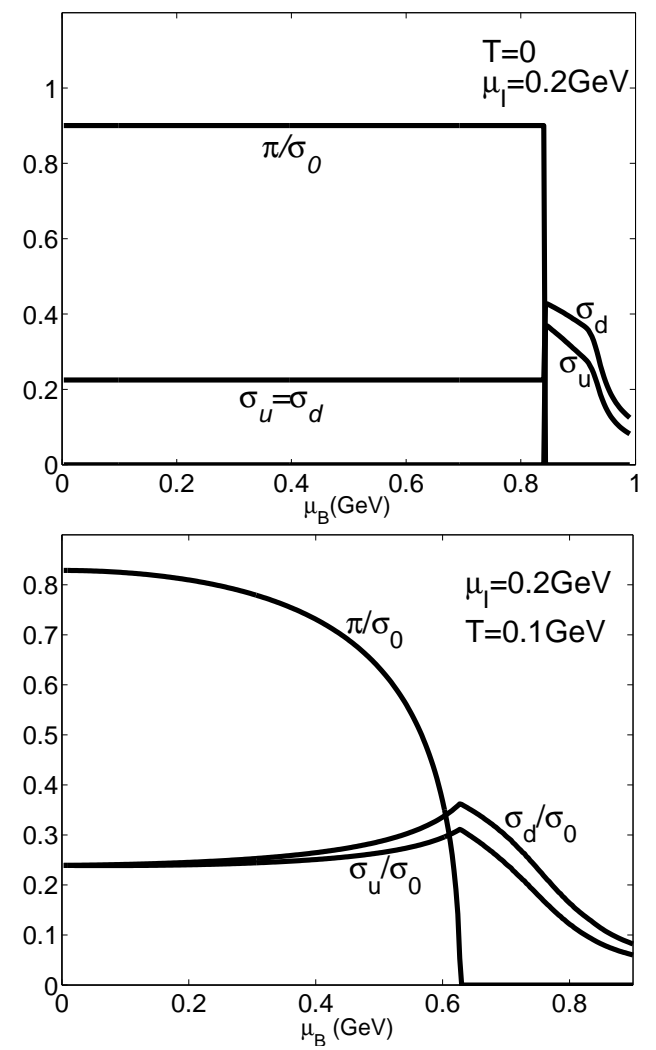

FIG. 19. The $u$ and $d$ quark condensates and pion condensate, scaled by the chiral condensate $\sigma_{0}$ in the vacuum, as functions of $\mu_{B}$ at $\mu_{I}=0.2 \mathrm{GeV}$ and $T=0$ (upper panel) and $T=0.1 \mathrm{GeV}$ (lower panel).

The temperature dependence of the three condensates is indicated in Fig.(20) at fixed chemical potentials. Similar to the case $[30,31]$ of investigating chiral symmetry restoration without pion condensation, the temperature effect here results in a second order phase transition of pion superfluidity. The phase diagram in the $T-\mu_{B}$ plane at fixed $\mu_{I}$ is shown in Fig.(21), which is very similar to the chiral phase transition line at $\mu_{I}=0$ [31]. The phase transition is of second order in high temperature region and of first order in high baryon chemical potential region, and the tricritical point which connects the first and second order phase transitions is located at $T=0.045 \mathrm{GeV}$ and $\mu_{B}=0.78 \mathrm{GeV}$.

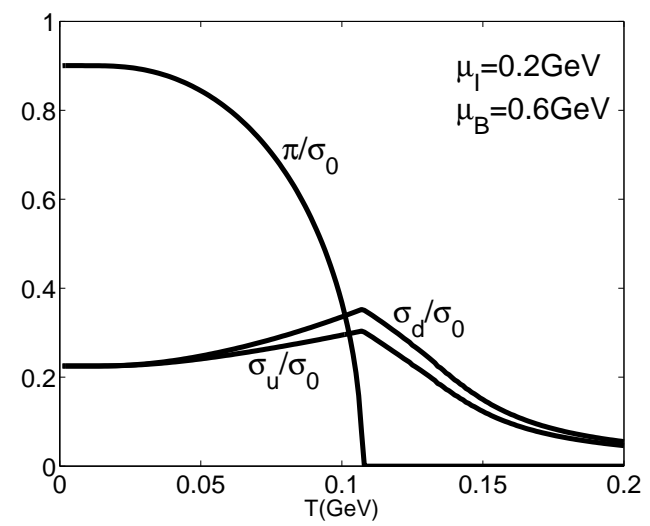

FIG. 20. The $u$ and $d$ quark condensates and pion condensate, scaled by the chiral condensate $\sigma_{0}$ in the vacuum, as functions of $T$ at $\mu_{I}=0.2 \mathrm{GeV}$ and $\mu_{B}=0.6 \mathrm{GeV}$.

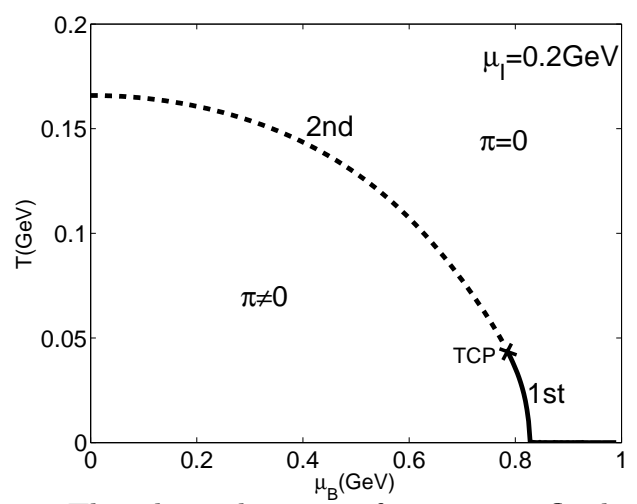

FIG. 21. The phase diagram of pion superfluidity in the $T-\mu_{B}$ plane at $\mu_{I}=0.2 \mathrm{GeV}$. The solid part of the line indicates a first order phase transition while the dashed part indicates one of second order. The symbol $\times$ is the position of the tricritical point.

\section{B. Competition between Pion and Diquark Condensates at $N_{c}=2$}

To study both pion superfluidity and color superconductivity at finite baryon and isospin chemical potentials, we first consider the two color NJL model with both quark-antiquark and diquark channels,

$$
\begin{aligned}
\mathcal{L} & =\bar{\psi}\left(i \gamma^{\mu} \partial_{\mu}-m_{0}\right) \psi+G_{S}\left((\bar{\psi} \psi)^{2}+\left(\bar{\psi} i \gamma_{5} \vec{\tau} \psi\right)^{2}\right) \\
& +G_{D}\left(\bar{\psi}^{c} i \gamma_{5} \tau_{2}^{c} \tau_{2}^{f} \psi\right)\left(\bar{\psi} i \gamma_{5} \tau_{2}^{c} \tau_{2}^{f} \psi^{c}\right)
\end{aligned}
$$

where $\tau_{2}^{c}$ and $\tau_{2}^{f}$ are the second Pauli matrix in color and flavor spaces, $G_{S}$ and $G_{D}$ are the coupling constants in the quark-antiquark channel and diquark channel, and from the Fierz transformation we have $G_{D}=G_{S}$ for $N_{c}=2$. 
At sufficiently small $\mu_{I}$ and large $\mu_{B}$, there should be only chiral condensate $\sigma$ and diquark condensate $\Delta$ defined as

$$
\Delta=\left\langle\bar{\psi}^{c} i \gamma_{5} \tau_{1}^{c} \tau_{1}^{f} \psi\right\rangle .
$$

Taking the similar way used in Section II, it is straightforward to derive the quark propagator matrix in NambuGorkov space and then obtain the gap equations for the two condensates $[34,35]$. At zero temperature they are

$$
\begin{aligned}
\sigma & +2 N_{c} M_{q} \int \frac{d^{3} \mathbf{k}}{(2 \pi)^{3}} \frac{1}{E_{k}}\left[\frac{E_{k}-\mu_{B} / N_{c}}{E_{\Delta}^{-}} \Theta\left(E_{\Delta}^{-}-\left|\mu_{I}\right| / 2\right)\right. \\
& \left.+\frac{E_{k}+\mu_{B} / N_{c}}{E_{\Delta}^{+}} \Theta\left(E_{\Delta}^{+}-\left|\mu_{I}\right| / 2\right)\right]=0 \\
\Delta & {\left[1-4 N_{c} G_{D} \int \frac{d^{3} \mathbf{k}}{(2 \pi)^{3}}\left(\frac{1}{E_{\Delta}^{-}} \Theta\left(E_{\Delta}^{-}-\left|\mu_{I}\right| / 2\right)\right.\right.} \\
& \left.\left.+\frac{1}{E_{\Delta}^{+}} \Theta\left(E_{\Delta}^{-}+\left|\mu_{I}\right| / 2\right)\right)\right]=0
\end{aligned}
$$

with the energy functions $E_{\Delta}^{ \pm}$and effective quark mass $M_{q}$ defined as

$$
\begin{aligned}
& E_{\Delta}^{ \pm}=\sqrt{\left(E_{k} \pm \mu_{B} / N_{c}\right)^{2}+4 G_{D}^{2} \Delta^{2}}, \\
& M_{q}=m_{0}-2 G_{S} \sigma .
\end{aligned}
$$

These gap equations for $\sigma$ and $\Delta$ are exactly the same as the gap equations for $\sigma$ and $\pi$ shown in Section II at $T=0$, if we do the replacement $\Delta \rightarrow \pi, \mu_{B} \rightarrow \mu_{I}$. Making comparison with the pion mass equation (37) in the vacuum and considering the symmetry between $\Delta$ and $\pi$, we obtain the critical baryon chemical potential $\mu_{B}^{c}$ of color superconductivity and the critical isospin chemical potential $\mu_{I}^{c}$ of pion superfluidity,

$$
\begin{aligned}
& \mu_{B}^{c}=m_{\pi}, \quad\left(\mu_{I}<m_{\pi}\right), \\
& \mu_{I}^{c}=m_{\pi}, \quad\left(\mu_{B}<m_{\pi}\right) .
\end{aligned}
$$

Therefore, the normal phase without pion and diquark condensations is in the square box $\mu_{I}<m_{\pi}$ and $\mu_{B}<$ $m_{\pi}$, shown in Fig.(22). The system is in the phase of color superconductivity with $\pi=0$ and $\Delta \neq 0$ for $\mu_{I}<$ $m_{\pi}$ and $\mu_{B}>m_{\pi}$ and in the phase of pion superfluidity with $\pi \neq 0$ and $\Delta=0$ for $\mu_{B}<m_{\pi}$ and $\mu_{I}>m_{\pi}$. In the region of high $\mu_{I}$ and $\mu_{B}$, due to the symmetry between the pion and diquark condensates proposed by their gap equations, we can conclude that the pion and diquark condensates are equivalent in the phase diagram in the $\mu_{I}-\mu_{B}$ plane. This can be confirmed by taking the bosonized version [42] of the two color NJL model with chiral, pion and diquark condensates. The effective potential in the bosonized version can be derived as

$$
\begin{aligned}
V(\sigma, \pi, \Delta) & =-a\left(\sigma^{2}+\pi^{2}+\Delta^{2}\right)+b\left(\sigma^{2}+\pi^{2}+\Delta^{2}\right)^{2} \\
& -c \sigma-\frac{1}{2} \mu_{I}^{2} \pi^{2}-\frac{1}{2} \mu_{B}^{2} \Delta^{2},
\end{aligned}
$$

where $a, b$ and $c$ are constants. We can easily obtain a first order phase transition line, shown by the dashed line in Fig.(22), by minimizing the effective potential. The phase diagram calculated here in the frame of NJL model is exactly the same as the one obtained with effective chiral Lagrangian for $N_{c}=2$ [13].
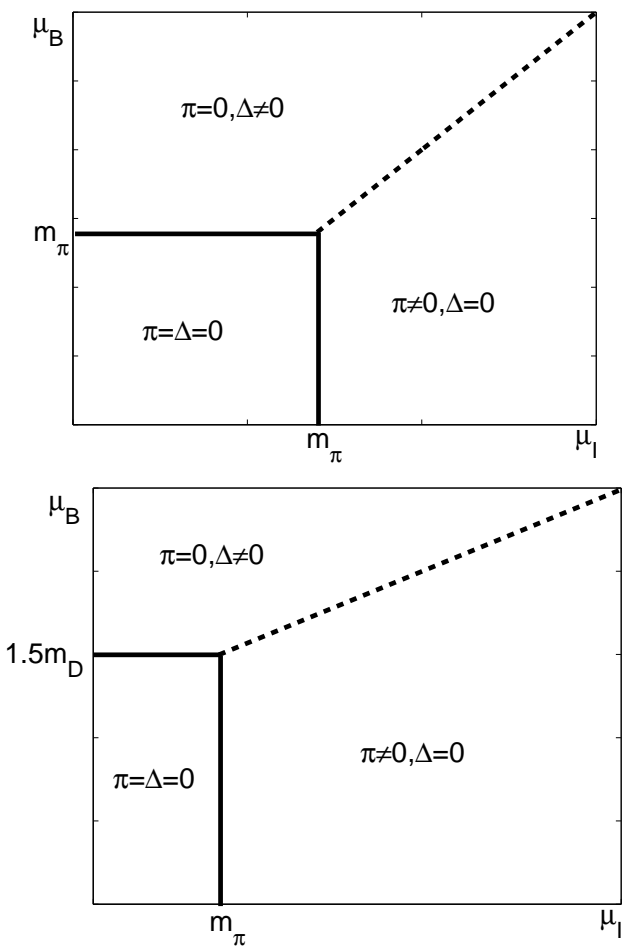

FIG. 22. The phase diagram of pion superfluidity and color superconductivity in the $\mu_{B}-\mu_{I}$ plane at $T=0$ in the frame of $N_{c}=2$ (upper panel) and $N_{c}=3$ (lower panel) NJL models.

\section{Competition between Pion and Diquark Condensations at $N_{c}=3$}

In the real world with color degrees of freedom $N_{c}=3$, the NJL model including the diquark sector is defined by the Lagrangian density

$$
\begin{aligned}
\mathcal{L} & =\bar{\psi}\left(i \gamma^{\mu} \partial_{\mu}-m_{0}\right) \psi+G_{S}\left((\bar{\psi} \psi)^{2}+\left(\bar{\psi} i \gamma_{5} \vec{\tau} \psi\right)^{2}\right) \\
& +G_{D}\left(\bar{\psi}_{i \alpha}^{c} i \gamma_{5} \epsilon_{c}^{\alpha \beta \gamma} \epsilon_{f}^{i j} \psi_{j \beta}\right)\left(\bar{\psi}_{i \alpha} i \gamma_{5} \epsilon_{c}^{\alpha \beta \gamma} \epsilon_{f}^{i j} \psi_{j \beta}^{c}\right)
\end{aligned}
$$

where $\epsilon_{f}^{i j}$ and $\epsilon_{c}^{\alpha \beta \gamma}$ are totally antisymmetric tensors in flavor and color spaces.

At sufficiently small $\mu_{I}$ and large $\mu_{B}$, there should be only chiral and diquark condensates. Taking the standard way used in Section II we can derive the quark propagator matrix in the Nambu-Gorkov space and then obtain the gap equations determining the chiral condensate $\sigma$ and diquark condensate $\Delta$ defined as

$$
\Delta=\left\langle\bar{\psi}_{i \alpha}^{c} \epsilon_{f}^{i j} \epsilon_{c}^{\alpha \beta 3} i \gamma_{5} \psi_{j \beta}\right\rangle,
$$


where it has been regarded that only the first two colors participate in the condensate, while the third one does not. At zero temperature, the $\mu_{B}$ and $\mu_{I}$ dependence of the two condensates are governed by the equations

$$
\begin{aligned}
\sigma+ & 4 M_{q} \int \frac{d^{3} \mathbf{k}}{(2 \pi)^{3}} \frac{1}{E_{k}}\left[\frac{E_{k}-\mu_{B} / 3}{E_{\Delta}^{-}} \Theta\left(E_{\Delta}^{-}-\left|\mu_{I}\right| / 2\right)\right. \\
& +\frac{E_{k}+\mu_{B} / 3}{E_{\Delta}^{+}} \Theta\left(E_{\Delta}^{+}-\left|\mu_{I}\right| / 2\right) \\
& \left.+\frac{1}{2} \Theta\left(E_{0}^{-}-\left|\mu_{I}\right| / 2\right)+\frac{1}{2} \Theta\left(E_{0}^{+}-\left|\mu_{I}\right| / 2\right)\right]=0, \\
\Delta & {\left[1-8 G_{D} \int \frac{d^{3} \mathbf{k}}{(2 \pi)^{3}}\left(\frac{1}{E_{\Delta}^{-}} \Theta\left(E_{\Delta}^{-}-\left|\mu_{I}\right| / 2\right)\right.\right.} \\
& \left.\left.+\frac{1}{E_{\Delta}^{+}} \Theta\left(E_{\Delta}^{+}-\left|\mu_{I}\right| / 2\right)\right)\right]=0 .
\end{aligned}
$$

Similar to the meson mass equations derived in RPA $[26,27,31]$, we can obtain the diquark mass in the vacuum by the pole equation

$$
1-8 G_{D} \int \frac{d^{3} \mathbf{k}}{(2 \pi)^{3}}\left(\frac{1}{E_{k}+m_{D} / 2}+\frac{1}{E_{k}-m_{D} / 2}\right)=0 .
$$

At sufficiently small $\mu_{B}$ and large $\mu_{I}$, the system should be in the pion superfluidity phase. The chiral and pion condensates $\sigma$ and $\pi$ are controlled by the gap equations shown in Section II. From the comparison of them at zero temperature with the pion mass equation in the vacuum, and the comparison of the gap equations (104) with the diquark mass equation (105), we get the conclusion that in the $\mu_{B}-\mu_{I}$ plane the normal phase without pion and diquark condensates is in the rectangle defined by

$$
\begin{aligned}
& \mu_{I}<m_{\pi}, \\
& \mu_{B}<\frac{3}{2} m_{D},
\end{aligned}
$$

shown in Fig.(22). Outside this rectangle, we know that the system is in color superconductivity phase with $\Delta \neq$ 0 and $\pi=0$ for $\mu_{I}<m_{\pi}$ and $\mu_{B}>\frac{3}{2} m_{D}$, and in pion superfluidity phase with $\pi \neq 0$ and $\Delta=0$ for $\mu_{B}<\frac{3}{2} m_{D}$ and $\mu_{I}>m_{\pi}$. As for the phase structure in the region with high baryon and isospin chemical potentials, it needs to consider the gap equations for the three condensates $\sigma, \pi$ and $\Delta$ simultaneously. The dashed line in the lower panel of Fig.(22) which separates the phase with $\pi=0$ and $\Delta \neq 0$ from the phase $\pi \neq 0$ and $\Delta=0$ is just an estimation of us.

\section{EXTENSION TO $S U(3)$ NJL MODEL}

We examine now the possible pion and kaon superfluidity at finite isospin and strangeness chemical potentials in the frame of flavor $S U(3)$ NJL model. The Lagrangian density is defined as [26-29]

$$
\begin{aligned}
\mathcal{L} & =\bar{\psi}\left(i \gamma^{\mu} \partial_{\mu}-m_{0}\right) \psi+G \sum_{a=0}^{8}\left[\left(\bar{\psi} \lambda_{a} \psi\right)^{2}+\left(\bar{\psi} i \gamma_{5} \lambda_{a} \psi\right)^{2}\right] \\
& -K\left[\operatorname{det} \bar{\psi}\left(1+\gamma_{5}\right) \psi+\operatorname{det} \bar{\psi}\left(1-\gamma_{5}\right) \psi\right]
\end{aligned}
$$

where $m_{0}=\operatorname{diag}\left(m_{0 u}, m_{0 d}, m_{0 s}\right)$ is the mass matrix of current quarks, $G$ and $K$ are coupling constants, and the t'Hooft's determinant includes six-fermion interaction. The three flavor NJL Lagrangian can be brought into an effective form similar to (5) of the two flavor NJL model by writing the six-fermion interaction in an effective four-body form [26-29],

$$
\begin{aligned}
\mathcal{L}_{e f f} & =\bar{\psi}\left(i \gamma^{\mu} \partial_{\mu}-m_{0}\right) \psi \\
& +\sum_{i=0}^{8}\left[G_{i}^{-}\left(\bar{\psi} \lambda^{i} \psi\right)^{2}+G_{i}^{+}\left(\bar{\psi} i \gamma_{5} \lambda^{i} \psi\right)^{2}\right] \\
& +\left[G_{03}^{-}\left(\bar{\psi} \lambda^{0} \psi\right)\left(\bar{\psi} \lambda^{3} \psi\right)+G_{03}^{+}\left(\bar{\psi} i \gamma_{5} \lambda^{0} \psi\right)\left(\bar{\psi} i \gamma_{5} \lambda^{3} \psi\right)\right] \\
& +\left[G_{30}^{-}\left(\bar{\psi} \lambda^{3} \psi\right)\left(\bar{\psi} \lambda^{0} \psi\right)+G_{30}^{+}\left(\bar{\psi} i \gamma_{5} \lambda^{3} \psi\right)\left(\bar{\psi} i \gamma_{5} \lambda^{0} \psi\right)\right] \\
& +\left[G_{08}^{-}\left(\bar{\psi} \lambda^{0} \psi\right)\left(\bar{\psi} \lambda^{8} \psi\right)+G_{08}^{+}\left(\bar{\psi} i \gamma_{5} \lambda^{0} \psi\right)\left(\bar{\psi} i \gamma_{5} \lambda^{8} \psi\right)\right] \\
& +\left[G_{80}^{-}\left(\bar{\psi} \lambda^{8} \psi\right)\left(\bar{\psi} \lambda^{0} \psi\right)+G_{80}^{+}\left(\bar{\psi} i \gamma_{5} \lambda^{8} \psi\right)\left(\bar{\psi} i \gamma_{5} \lambda^{0} \psi\right)\right] \\
& +\left[G_{38}^{-}\left(\bar{\psi} \lambda^{3} \psi\right)\left(\bar{\psi} \lambda^{8} \psi\right)+G_{38}^{+}\left(\bar{\psi} i \gamma_{5} \lambda^{3} \psi\right)\left(\bar{\psi} i \gamma_{5} \lambda^{8} \psi\right)\right] \\
& +\left[G_{83}^{-}\left(\bar{\psi} \lambda^{8} \psi\right)\left(\bar{\psi} \lambda^{3} \psi\right)+G_{83}^{+}\left(\bar{\psi} i \gamma_{5} \lambda^{8} \psi\right)\left(\bar{\psi} i \gamma_{5} \lambda^{3} \psi\right)\right]
\end{aligned}
$$

with the effective couplings

$$
\begin{aligned}
& G_{0}^{ \pm}=G \mp \frac{1}{3} N_{c} K\left(\sigma_{u}+\sigma_{d}+\sigma_{s}\right) \\
& G_{1}^{ \pm}=G_{2}^{ \pm}=G_{3}^{ \pm}=G \pm \frac{1}{2} N_{c} K \sigma_{s} \\
& G_{4}^{ \pm}=G_{5}^{ \pm}=G \pm \frac{1}{2} N_{c} K \sigma_{d} \\
& G_{6}^{ \pm}=G_{7}^{ \pm}=G \pm \frac{1}{2} N_{c} K \sigma_{u}, \\
& G_{8}^{ \pm}=G \pm \frac{1}{6} N_{c} K\left(2 \sigma_{u}+2 \sigma_{d}-\sigma_{s}\right) \\
& G_{03}^{ \pm}=G_{30}^{ \pm}=\mp \frac{1}{2 \sqrt{6}} N_{c} K\left(\sigma_{u}-\sigma_{d}\right) \\
& G_{08}^{ \pm}=G_{80}^{ \pm}= \pm \frac{\sqrt{2}}{12} N_{c} K\left(\sigma_{u}+\sigma_{d}-2 \sigma_{s}\right) \\
& G_{38}^{ \pm}=G_{83}^{ \pm}= \pm \frac{1}{2 \sqrt{3}} N_{c} K\left(\sigma_{u}-\sigma_{d}\right)
\end{aligned}
$$

where $\sigma_{s}=\langle\bar{s} s\rangle$ is the $s$ quark condensate. The key thermodynamic quantity, the partition function $Z$ in three flavor case, is defined by

$$
Z\left(T, \mu_{I}, \mu_{B}, \mu_{S}, V\right)=\operatorname{Tr} e^{-\beta\left(H-\mu_{B} B-\mu_{I} I_{3}-\mu_{S} S\right)},
$$

with baryon number, isospin number and strangeness number 


$$
\begin{aligned}
& B=\frac{1}{3} \int d^{3} \mathbf{x} \bar{\psi} \gamma^{0} \psi, \\
& I_{3}=\frac{1}{2} \int d^{3} \mathbf{x} \bar{\psi} \gamma^{0} \lambda_{3} \psi, \\
& S=-\int d^{3} \mathbf{x} \bar{s} \gamma^{0} s,
\end{aligned}
$$

as conserved charges, and baryon, isospin and strangeness chemical potentials $\mu_{B}, \mu_{I}$ and $\mu_{S}$. In the frame of imaginary time formulism of finite temperature field theory, the partition function can be represented as

$$
\begin{aligned}
& Z\left(T, \mu_{I}, \mu_{B}, \mu_{S}, V\right)= \\
& \quad \int[d \bar{\psi}][d \psi] e^{\int_{0}^{\beta} d \tau \int d^{3} \mathbf{x}\left(\mathcal{L}_{e f f}+\bar{\psi} \mu \gamma_{0} \psi\right),}
\end{aligned}
$$

where $\mu=\operatorname{diag}\left(\mu_{u}, \mu_{d}, \mu_{s}\right)$ is the chemical potential matrix in flavor space with the chemical potential for the strangeness quark

$$
\mu_{s}=\frac{\mu_{B}}{3}-\mu_{S}
$$

\section{A. Chiral Properties at Low $\mu_{I}$ and $\mu_{S}$}

We discuss first the three chiral condensates $\sigma_{u}, \sigma_{d}$ and $\sigma_{s}$ under the condition of low isospin and low strangeness chemical potentials, $\mu_{I}<\mu_{I}^{c}$ and $\mu_{S}<\mu_{S}^{c}$, where $\mu_{I}^{c}$ is the critical isospin chemical potential for pion condensation and $\mu_{S}^{c}$ the critical strangeness chemical potential for kaon condensation. They will be determined in the following subsection. Performing the standard mean field approach and keeping only the linear terms in the meson fluctuations, we obtain the Lagrangian in the mean field approximation

$$
\begin{aligned}
\mathcal{L}_{m f} & =\bar{\psi}\left(i \gamma^{\mu} \partial_{\mu}+\mu \gamma_{0}-M\right) \psi \\
& -2 G\left(\sigma_{u}^{2}+\sigma_{d}^{2}+\sigma_{s}^{2}\right)-4 K \sigma_{u} \sigma_{d} \sigma_{s},
\end{aligned}
$$

where $M=\left(M_{u}, M_{d}, M_{s}\right)$ is the mass matrix in flavor space with the effective quark masses

$$
\begin{aligned}
& M_{i}=m_{0 i}-4 G \sigma_{i}+2 K \sigma_{j} \sigma_{k}, \\
& \quad(i=u, d, s, i \neq j \neq k, j<k) .
\end{aligned}
$$

Because of the lack of pion and kaon condensates, the mean field quark propagator is diagonal in flavor space,

$$
\mathcal{S}_{m f}(k)=\operatorname{diag}\left(\mathcal{S}_{u}(k), \mathcal{S}_{d}(k), \mathcal{S}_{s}(k)\right),
$$

with the matrix elements

$$
\mathcal{S}_{i}(k)=\frac{\Lambda_{+}^{i} \gamma_{0}}{k_{0}-E_{i}^{-}(\mathbf{k})}+\frac{\Lambda_{-}^{i} \gamma_{0}}{k_{0}+E_{i}^{+}(\mathbf{k})},
$$

where $E_{i}^{ \pm}$are the effective quark energies

$$
E_{i}^{ \pm}(\mathbf{k})=\sqrt{\mathbf{k}^{2}+M_{i}^{2}} \pm \mu_{i}
$$

and $\Lambda_{ \pm}^{i}$ the energy projectors

$$
\Lambda_{ \pm}^{i}=\frac{1}{2}\left(1 \pm \frac{\gamma_{0}\left(\gamma \cdot \mathbf{k}+M_{i}\right)}{\sqrt{\mathbf{k}^{2}+M_{i}^{2}}}\right) .
$$

In self-consistent Hatree-Fock approximation the gap equations which determine the value of the chiral condensates $\sigma_{u}, \sigma_{d}, \sigma_{s}$ are expressed in terms of the quark propagators,

$$
\sigma_{i}=-i N_{c} \int \frac{d^{4} p}{(2 \pi)^{4}} \operatorname{Tr}_{D} \mathcal{S}_{i}(p)
$$

After performing the Matsubara frequency summation, we have

$$
\sigma_{i}=-6 \int \frac{d^{3} \mathbf{k}}{(2 \pi)^{3}} \frac{M_{i}}{\sqrt{k^{2}+M_{i}^{2}}}\left(1-f\left(E_{i}^{-}\right)-f\left(E_{i}^{+}\right)\right) .
$$

The three effective quark masses are shown in Fig.(23) as functions of baryon chemical potential $\mu_{B}$ at $T=\mu_{S}=0$ and $\mu_{I}=0.06 \mathrm{GeV}<m_{\pi}$. We use the model parameters fixed in [46]. The three masses keep their vacuum values till the common critical point where they suddenly jump down. The mass difference between $u$ and $d$ quarks arisen from the finite isospin chemical potential can only be seen after the chiral transition.

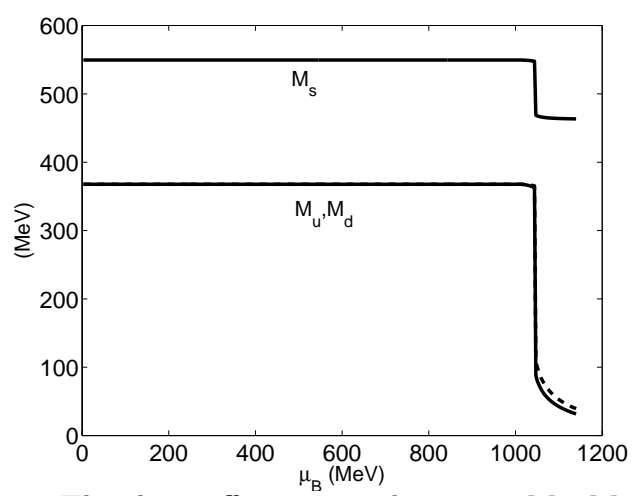

FIG. 23. The three effective quark masses $M_{u}, M_{d}$ and $M_{s}$ as functions of baryon chemical potential $\mu_{B}$ at $T=\mu_{S}=0$ and $\mu_{I}=0.06 \mathrm{GeV}$ in the flavor $\mathrm{SU}(3)$ NJL model.

In principle the meson mass spectra can be evaluated by solving the corresponding pole equation like (54) for two flavor NJL model. The meson polarization functions in flavor space are evaluated in Appendix D. In general case with nonzero pion and kaon condensates, the off diagonal elements in the polarization function matrix in flavor space makes it complicated to solve the pole equation. However, in the region without pion and kaon condensates we are interested in here, the off diagonal elements disappear and the pole equation is reduced to 


$$
1-2 G_{i}^{ \pm} \Pi_{M M}\left(k_{0}=M_{M}, \mathbf{k}=\mathbf{0}\right)=0,
$$

with $G_{1}^{-}$for $M=a^{0}, a^{+}, a^{-}, G_{4}^{-}$for $M=\kappa^{+}, \kappa^{-}, G_{6}^{-}$for $\kappa^{0}, \bar{\kappa}^{0}, G_{1}^{+}$for $M=\pi^{0}, \pi^{+}, \pi^{-}, G_{4}^{+}$for $M=K^{+}, K^{-}$and $G_{6}^{+}$for $K^{0}, \bar{K}^{0}$. At zero temperature, we can analytically obtain the meson masses as functions of $\mu_{I}$ and $\mu_{S}$ by comparing the above mass equations at finite $\mu_{I}$ and $\mu_{S}$ with the same equations but in the vacuum,

$$
M_{M}\left(\mu_{I}, \mu_{S}\right)=m_{M}-Q_{I} \mu_{I}-Q_{S} \mu_{S},
$$

where $Q_{I}$ and $Q_{S}$ are the isospin number and strangeness number of the meson $M$, respectively.

\section{B. Phase Diagram in $\mu_{S}-\mu_{I}$ plane}

In this subsection, we analytically determine the critical isospin chemical potential $\mu_{I}^{c}$ for pion condensation and the critical strangeness chemical potential $\mu_{S}^{c}$ for kaon condensation in the three flavor NJL model, and briefly discuss the phase structure in $\mu_{S}-\mu_{I}$ plane. For simplicity, we always set $T=\mu_{B}=0$ in this subsection.

We first consider the case with only pion condensation in the region of $\left|\mu_{S}\right|<\mu_{S}^{C}$ and determine the critical value $\mu_{I}^{c}$. Taking the standard mean field approximation, we obtain the inverse of the quark propagator

$$
\mathcal{S}_{m f}^{-1}(k)=\left(\begin{array}{ccc}
G_{u}^{-1} & 2 i G_{1}^{+} \gamma_{5} \pi & 0 \\
2 i G_{1}^{+} \gamma_{5} \pi & G_{d}^{-1} & 0 \\
0 & 0 & G_{s}^{-1}
\end{array}\right),
$$

with $G_{i}^{-1}=\gamma^{\mu} k_{\mu}+\mu_{i} \gamma_{0}-M_{i}$ for $i=u, d$, s. Since in the current case the pion condensate is decoupled from the $s$ quark propagator, we can take the same procedure as in the two flavor case and obtain the same critical isospin chemical potential

$$
\mu_{I}^{c}=m_{\pi} \quad\left(\mu_{S}<\mu_{S}^{c}\right) .
$$

We then study the case with only kaon condensate in the region of $\mu_{I}<m_{\pi}$. We define the $K^{+}$and $K^{-}$condensates

$$
K_{u s}=2\left\langle\bar{u} i \gamma_{5} s\right\rangle=2\left\langle\bar{s} i \gamma_{5} u\right\rangle,
$$

and $K^{0}, \bar{K}^{0}$ condensates

$$
K_{d s}=2\left\langle\bar{d} i \gamma_{5} s\right\rangle=2\left\langle\bar{s} i \gamma_{5} d\right\rangle .
$$

Which kind of kaon condensation happens depends on the sign of $\mu_{S}$ and $\mu_{I}$. In the physical world with $\mu_{I}<0$, only $K^{0}$ and $K^{-}$condensates can be realized. Without losing generality, we consider here the case $\mu_{I}>0$ and $\mu_{S}>0$ only, and then map the result to the other regions in the $\mu_{S}-\mu_{I}$ plane. In this case the possible kaon condensation is $K^{+}$.
At mean field level the inverse quark propagator in the flavor space can be expressed as

$$
\mathcal{S}_{m f}^{-1}(k)=\left(\begin{array}{ccc}
G_{u}^{-1} & 2 i G_{4}^{+} K_{u s} \gamma_{5} & 0 \\
2 i G_{4}^{+} K_{u s} \gamma_{5} & G_{s}^{-1} & 0 \\
0 & 0 & G_{d}^{-1}
\end{array}\right) .
$$

For convenience, we have changed the basis in the flavor space from $(u, d, s)$ in $(124)$ to $(u, s, d)$ in (128). It is straightforward to derive the propagator itself from its inverse,

$$
\mathcal{S}_{m f}(k)=\left(\begin{array}{ccc}
\mathcal{S}_{u}(k) & \mathcal{S}_{u s}(k) & 0 \\
\mathcal{S}_{s u}(k) & \mathcal{S}_{s}(k) & 0 \\
0 & 0 & \mathcal{S}_{d}(k)
\end{array}\right),
$$

with the elements

$$
\begin{aligned}
\mathcal{S}_{u}(k) & =\left[\left(\gamma^{\mu} k_{\mu}+\mu_{u} \gamma_{0}-M_{u}\right)\right. \\
& \left.+4\left(G_{4}^{+}\right)^{2} K_{u s}^{2} \gamma_{5}\left(\gamma^{\mu} k_{\mu}+\mu_{s} \gamma_{0}-M_{s}\right)^{-1} \gamma_{5}\right]^{-1}, \\
\mathcal{S}_{d}(k) & =\left(\gamma^{\mu} k_{\mu}+\mu_{d} \gamma_{0}-M_{d}\right)^{-1} \\
\mathcal{S}_{s}(k) & =\left[\left(\gamma^{\mu} k_{\mu}+\mu_{s} \gamma_{0}-M_{s}\right)\right. \\
& \left.+4\left(G_{4}^{+}\right)^{2} K_{u s}^{2} \gamma_{5}\left(\gamma^{\mu} k_{\mu}+\mu_{u} \gamma_{0}-M_{u}\right)^{-1} \gamma_{5}\right]^{-1}, \\
\mathcal{S}_{u s}(k) & =-2 i G_{4}^{+} K_{u s} \mathcal{S}_{u}(k) \gamma_{5}\left(\gamma^{\mu} k_{\mu}+\mu_{s} \gamma_{0}-M_{s}\right)^{-1}, \\
\mathcal{S}_{s u}(k) & =-2 i G_{4}^{+} K_{u s} \mathcal{S}_{s}(k) \gamma_{5}\left(\gamma^{\mu} k_{\mu}+\mu_{u} \gamma_{0}-M_{u}\right)^{-1}
\end{aligned}
$$

With the propagator we can express the condensates as

$$
\begin{aligned}
\sigma_{i} & =-N_{c} \int \frac{d^{4} k}{(2 \pi)^{4}} \operatorname{Tr}_{D}\left[i\left(\mathcal{S}_{i}(k)\right], \quad(i=u, d, s)\right. \\
K_{u s} & =N_{c} \int \frac{d^{4} k}{(2 \pi)^{4}} \operatorname{Tr}_{D}\left[\left(\mathcal{S}_{u s}(k)+\mathcal{S}_{s u}(k)\right) \gamma_{5}\right] .
\end{aligned}
$$

To calculate the critical strangeness chemical potential, we set $K_{u s} \rightarrow 0^{+}$, and the gap equations on the phase transition line become

$$
\begin{aligned}
& \sigma_{i}+i N_{c} \int \frac{d^{4} k}{(2 \pi)^{4}} \operatorname{Tr}_{D} \frac{1}{\gamma^{\mu} k_{\mu}+\mu_{i} \gamma_{0}-M_{i}}=0, \\
& 1+2 N_{c} i G_{4}^{+} \int \frac{d^{4} k}{(2 \pi)^{4}} \\
& \operatorname{Tr}_{D}\left[\frac{1}{\gamma^{\mu} k_{\mu}+\mu_{u} \gamma_{0}-M_{u}} \gamma_{5} \frac{1}{\gamma^{\mu} k_{\mu}+\mu_{s} \gamma_{0}-M_{s}} \gamma_{5}\right]=0 .
\end{aligned}
$$

Doing the trace in Dirac space, performing the Matsubara frequency summation, and then comparing the gap equations with the mass equations for kaons in the vacuum, we find

$$
\mu_{S}^{c}=m_{K}-\mu_{I} / 2, \quad\left(\mu_{I}<m_{\pi}\right) .
$$

This result is certainly independent of the model parameters and the regularization scheme. The above analytic 
conclusions $\mu_{I}^{c}=m_{\pi}$ and $\mu_{S}^{c}=m_{K}-\mu_{I} / 2$ were obtained numerically in the three flavor NJL model without $U_{A}(1)$ breaking term [18].
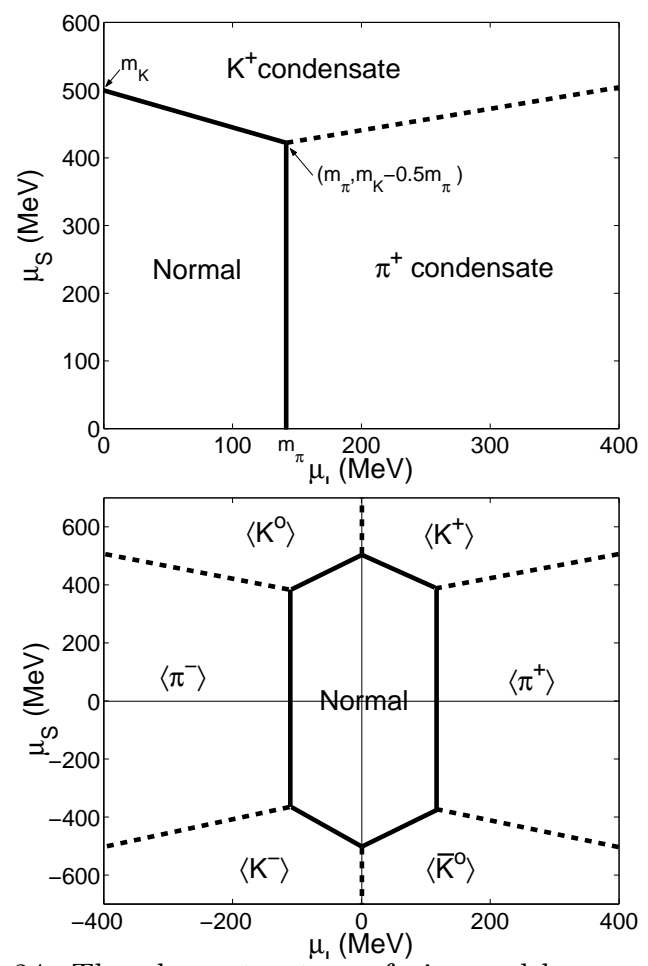

FIG. 24. The phase structure of pion and kaon condensations in $\mu_{S}-\mu_{I}$ plane at $T=\mu_{B}=0$ in the flavor $S U(3)$ NJL model. The upper panel is for $\mu_{I}>0$ and $\mu_{S}>0$ and the lower panel is for all possible $\mu_{I}$ and $\mu_{S}$.

Combining the two critical chemical potentials $\mu_{I}$ and $\mu_{S}$, the system is in the normal phase without pion and kaon condensations when $\mu_{I}$ and $\mu_{S}$ satisfy the constraints

$$
\begin{aligned}
& \mu_{I}<m_{\pi}, \\
& \mu_{S}<m_{K}-\mu_{I} / 2 .
\end{aligned}
$$

In the region of $\mu_{I}<m_{\pi}$ and $\mu_{S}>m_{K}-\mu_{I} / 2$, there is only $K^{+}$condensation, and in the region of $\mu_{S}<m_{K}-\mu_{I} / 2$ and $\mu_{I}>m_{\pi}$ there is only pion condensation. The phase transition lines in the $\mu_{S}-\mu_{I}$ plane are indicated by solid lines in Fig.(24). As for the other region in the $\mu_{S}-\mu_{I}$ plane we should consider both pion and kaon condensations and the calculation will become much complicated. The dashed line in Fig.(24) which separates the region with pion condensate from the region with kaon condensate is just an estimation. However, with the bosonized chiral Lagrangian, this dashed line is described by [12]

$\mu_{S}^{C}\left(\left|\mu_{I}\right|>m_{\pi}\right)=\frac{1}{2 \mu_{I}}\left[\sqrt{\left(\mu_{I}^{2}-m_{\pi}^{2}\right)^{2}+4 m_{K}^{2} \mu_{I}^{2}}-m_{\pi}^{2}\right]$.
Considering the symmetries between $\pi_{+}$and $\pi_{-}$and among $K^{+}, K^{-}, K^{0}$ and $\bar{K}^{0}$, we display in the lower panel of Fig.(24) the phase diagram with different pion and kaon condensations, which is the same as the one obtained in the frame of bosonized chiral lagrangian [12].

\section{SUMMARY}

It is well-known that physical symmetries of a system dominate its behavior not only in the vacuum but also in hot and dense medium. The symmetries in the vacuum are usually changed by the temperature and density effect. In this paper we have investigated the changes of isospin symmetry, chiral symmetry and color symmetry and their reflection in meson spectra and thermodynamic functions at finite temperature $T$ and isospin, baryon and strangeness chemical potentials $\mu_{I}, \mu_{B}$ and $\mu_{S}$ in effective models.

We studied the flavor $S U(2)$ NJL model in detail. With the standard technics we set up in flavor space the quark propagator matrix with off diagonal elements arisen from the pion condensation at finite isospin density. With the propagator we derived the gap equations determining the temperature and chemical potential dependence of the pion and chiral condensates, and obtained the thermodynamic functions in mean field approximation. By self-consistently solving the gap equations at finite isospin density and the pion mass equation in the vacuum, we analytically derived the critical isospin chemical potential for pion superfluidity, $\mu_{I}^{c}=m_{\pi}$, at quark level. Therefore, in chiral limit with vanishing pion mass, any small isospin effect will lead to chiral symmetry restoration and isospin symmetry spontaneous breaking even in the vacuum. In real world with finite current quark mass and at finite temperature, there is a strong competition between the pion and chiral condensates in their coexistence region. The chiral condensate goes up continuously in the mixed region and drops down outside, while the pion condensate decreases monotonously in the whole temperature region. The bulk properties of the system in the limit of high isospin density is also quite different from the corresponding ideal gas, the equation of state is hard and the ration of pressure to energy density approaches 0.7. We investigated the effect of pion superfluidity on the meson properties in the hot and dense quark-meson plasma, by taking into account the off diagonal elements in the meson polarization function matrix in isospin space. We found that the mean field approximation to quarks together with RPA to mesons can well describe the isospin spontaneous breaking, there is a massless Goldstone mode which is a linear combination of the $\pi_{+}, \pi_{-}$and $\sigma$ modes in the whole superfluidity phase. We proved the Goldstone mode analytically in general case with finite $T, \mu_{I}$ and $\mu_{B}$. In the superfluidity phase, while the $\pi_{0}$ mode is still an eigen mode, the $\sigma, \pi_{+}$ 
and $\pi_{-}$mixing is very strong, especially in the region close to the phase transition point. We discussed also the competition between the pion superfluidity and color superconductivity at finite baryon density. While we did not make full calculation with both pion and diquark condensates, we determined the critical baryon chemical potential $\mu_{B}^{c}$ for color superconductivity. The region of the normal phase without pion and diquark condensates in $\mu_{S}-\mu_{I}$ plane is controlled by the pion mass $m_{\pi}$ and diquark mass $m_{D}$ in the vacuum. In the case of two colors it is in a square box defined by $\mu_{I}<m_{\pi}$ and $\mu_{B}<m_{\pi}$, and in the case of three colors it is in a rectangle defined by $\mu_{I}<m_{\pi}$ and $\mu_{B}<1.5 m_{D}$.

We briefly extended our discussion on pion superfluidity to the flavor $S U(3)$ NJL model at finite isospin and strangeness densities. We found that the conclusion of the critical isospin chemical potential $\mu_{I}^{c}=m_{\pi}$ for pion superfluidity does not change, and that the critical strangeness chemical potential for kaon superfluidity is related not only to the kaon mass $m_{K}$ but also to the isospin chemical potential, $\mu_{S}^{c}=m_{K}-\mu_{I} / 2$. We showed the phase diagram of pion and kaon superfluidity in $\mu_{S}-\mu_{I}$ plane with possible charged pion condensates and kaon condensates.

We have investigated the pion superfluidity and meson properties at tree level and the thermal excitation at finite isospin density in the bosonized version of the NJL model, namely, the linear sigma model, and made comparison of our calculations in the NJL model and sigma model with the result obtained in chiral perturbation theory. Many conclusions in the linear sigma model are qualitatively or even quantitatively consistent with that in the NJL model. We found that at zero temperature the linear sigma model can reproduce the result of chiral perturbation theory by taking the limit $m_{\sigma} \rightarrow \infty$, but at finite temperature the Hartree-Fock approximation can not recover the Goldstone mode in the pion superfluidity phase. The phase diagram in the $T-\mu_{I}$ plane in large $N$ expansion approach is more reasonable than the one in the Hartree-Fock approximation.

It is necessary to discuss the difference among the effective models. The conclusion $\mu_{I}^{c}=m_{\pi}$ is observed in almost all model calculations and lattice simulation. While obtaining this result is easy and looks trivial in the meson effective models such as chiral perturbation theory and linear sigma model, because the pion mass is a parameter in these models, it is in the NJL model a general result of mean field approximation to quarks and RPA to mesons and diquarks. This is also true for kaon condensation. In chiral perturbation theory, the chiral and pion condensates are regarded as a rotation from each other. As a result, the maximum pion condensate should be the same as the chiral condensate in the vacuum. In the NJL model, we found that this rotation point of view is only true around the phase transition point. Beyond this region the pion condensate can be larger than the maximal chiral condensate in the vacuum. This behavior is qualitatively consistent with the lattice simulation [9-11]. As for the linear sigma model, because the pion condensate increases with $\mu_{I}$ without bound, the phase structure at high $\mu_{I}$ is unphysical. Of course, due to the lack of confinement mechanism in the NJL model, we need a momentum cutoff $\Lambda$ in numerical calculations. Therefore, we can not take the numerical results in high temperature and high density regions seriously.

Acknowledgments: The work was supported in part by the grants NSFC10135030, 10425810, 10435080, G2000077407 and SRFDP20040003103.

\section{APPENDIX A: TRACE IN DIRAC SPACE}

In obtaining the gap equations for the condensates and the meson and diquark polarization functions, we need to take the trace of the quark propagators including energy projectors in Dirac space. Here we list the results.

$$
\begin{aligned}
& \operatorname{Tr}\left[\Lambda_{+}(\mathbf{p}) \gamma_{0} \Lambda_{+}(\mathbf{p}) \gamma_{0}\right]=\operatorname{Tr}\left[\Lambda_{-}(\mathbf{p}) \gamma_{0} \Lambda_{-}(\mathbf{p}) \gamma_{0}\right] \\
& =2 M^{2} / E_{p}^{2}, \\
& \operatorname{Tr}\left[\Lambda_{+}(\mathbf{p}) \gamma_{0} \Lambda_{-}(\mathbf{p}) \gamma_{0}\right]=\operatorname{Tr}\left[\Lambda_{-}(\mathbf{p}) \gamma_{0} \Lambda_{+}(\mathbf{p}) \gamma_{0}\right] \\
& =2-2 M^{2} / E_{p}^{2}, \\
& \operatorname{Tr}\left[\Lambda_{+}(\mathbf{p}) \gamma_{5} \Lambda_{+}(\mathbf{p}) \gamma_{5}\right]=\operatorname{Tr}\left[\Lambda_{-}(\mathbf{p}) \gamma_{5} \Lambda_{-}(\mathbf{p}) \gamma_{5}\right] \\
& =2-2 M^{2} / E_{p}^{2}, \\
& \operatorname{Tr}\left[\Lambda_{+}(\mathbf{p}) \gamma_{5} \Lambda_{-}(\mathbf{p}) \gamma_{5}\right]=\operatorname{Tr}\left[\Lambda_{-}(\mathbf{p}) \gamma_{5} \Lambda_{+}(\mathbf{p}) \gamma_{5}\right] \\
& =2 M^{2} / E_{p}^{2}, \\
& \operatorname{Tr}\left[\Lambda_{e}(\mathbf{p}) \gamma_{0} \Lambda_{e^{\prime}}(\mathbf{p}) \gamma_{5}\right]=\operatorname{Tr}\left[\Lambda_{e}(\mathbf{p}) \gamma_{5} \Lambda_{e^{\prime}}(\mathbf{p}) \gamma_{0}\right] \\
& =0 \text {, } \\
& \operatorname{Tr}\left[\gamma_{5} \Lambda_{+}(\mathbf{p}) \gamma_{0} \gamma_{5} \Lambda_{+}(\mathbf{p}) \gamma_{0}\right]=\operatorname{Tr}\left[\gamma_{5} \Lambda_{-}(\mathbf{p}) \gamma_{0} \gamma_{5} \Lambda_{-}(\mathbf{p}) \gamma_{0}\right] \\
& =0 \text {, } \\
& \operatorname{Tr}\left[\gamma_{5} \Lambda_{+}(\mathbf{p}) \gamma_{0} \gamma_{5} \Lambda_{-}(\mathbf{p}) \gamma_{0}\right]=\operatorname{Tr}\left[\gamma_{5} \Lambda_{-}(\mathbf{p}) \gamma_{0} \gamma_{5} \Lambda_{+}(\mathbf{p}) \gamma_{0}\right] \\
& =-2 \text {, } \\
& \operatorname{Tr}\left[\gamma_{5} \Lambda_{+}(\mathbf{p}) \gamma_{5} \gamma_{5} \Lambda_{+}(\mathbf{p}) \gamma_{5}\right]=\operatorname{Tr}\left[\gamma_{5} \Lambda_{-}(\mathbf{p}) \gamma_{5} \gamma_{5} \Lambda_{-}(\mathbf{p}) \gamma_{5}\right] \\
& =2 \text {, } \\
& \operatorname{Tr}\left[\gamma_{5} \Lambda_{+}(\mathbf{p}) \gamma_{5} \gamma_{5} \Lambda_{-}(\mathbf{p}) \gamma_{5}\right]=\operatorname{Tr}\left[\gamma_{5} \Lambda_{-}(\mathbf{p}) \gamma_{5} \gamma_{5} \Lambda_{+}(\mathbf{p}) \gamma_{5}\right] \\
& =0 \text {, } \\
& \operatorname{Tr}\left[\gamma_{5} \Lambda_{e}(\mathbf{p}) \gamma_{0} \gamma_{5} \Lambda_{e^{\prime}}(\mathbf{p}) \gamma_{5}\right]=\operatorname{Tr}\left[\gamma_{5} \Lambda_{e}(\mathbf{p}) \gamma_{5} \gamma_{5} \Lambda_{e^{\prime}}(\mathbf{p}) \gamma_{0}\right] \\
& =0 \text {, } \\
& \operatorname{Tr}\left[\Lambda_{e}(\mathbf{p}) \gamma_{0} \gamma_{5} \Lambda_{e^{\prime}}(\mathbf{p}) \gamma_{0}\right]=\operatorname{Tr}\left[\gamma_{5} \Lambda_{e}(\mathbf{p}) \gamma_{0} \Lambda_{e^{\prime}}(\mathbf{p}) \gamma_{0}\right] \\
& =0 \text {, } \\
& \operatorname{Tr}\left[\Lambda_{e}(\mathbf{p}) \gamma_{5} \gamma_{5} \Lambda_{e^{\prime}}(\mathbf{p}) \gamma_{5}\right]=\operatorname{Tr}\left[\gamma_{5} \Lambda_{e}(\mathbf{p}) \gamma_{5} \Lambda_{e^{\prime}}(\mathbf{p}) \gamma_{5}\right] \\
& =0 \text {, } \\
& \operatorname{Tr}\left[\Lambda_{+}(\mathbf{p}) \gamma_{0} \gamma_{5} \Lambda_{+}(\mathbf{p}) \gamma_{5}\right]=\operatorname{Tr}\left[\Lambda_{-}(\mathbf{p}) \gamma_{0} \gamma_{5} \Lambda_{-}(\mathbf{p}) \gamma_{5}\right] \\
& =0 \text {, } \\
& \operatorname{Tr}\left[\Lambda_{+}(\mathbf{p}) \gamma_{0} \gamma_{5} \Lambda_{-}(\mathbf{p}) \gamma_{5}\right]=2 M / E_{p}, \\
& \operatorname{Tr}\left[\Lambda_{-}(\mathbf{p}) \gamma_{0} \gamma_{5} \Lambda_{+}(\mathbf{p}) \gamma_{5}\right]=-2 M / E_{p} \text {, }
\end{aligned}
$$




$$
\begin{aligned}
\operatorname{Tr}\left[\Lambda_{+}(\mathbf{p}) \gamma_{5} \gamma_{5} \Lambda_{-}(\mathbf{p}) \gamma_{0}\right] & =\operatorname{Tr}\left[\Lambda_{-}(\mathbf{p}) \gamma_{5} \gamma_{5} \Lambda_{+}(\mathbf{p}) \gamma_{0}\right] \\
& =0, \\
\operatorname{Tr}\left[\Lambda_{+}(\mathbf{p}) \gamma_{5} \gamma_{5} \Lambda_{+}(\mathbf{p}) \gamma_{0}\right] & =2 M / E_{p}, \\
\operatorname{Tr}\left[\Lambda_{-}(\mathbf{p}) \gamma_{5} \gamma_{5} \Lambda_{-}(\mathbf{p}) \gamma_{0}\right] & =-2 M / E_{p}, \\
\operatorname{Tr}\left[\gamma_{5} \Lambda_{+}(\mathbf{p}) \gamma_{5} \Lambda_{+}(\mathbf{p}) \gamma_{0}\right] & =\operatorname{Tr}\left[\gamma_{5} \Lambda_{-}(\mathbf{p}) \gamma_{5} \Lambda_{-}(\mathbf{p}) \gamma_{0}\right] \\
& =0, \\
\operatorname{Tr}\left[\gamma_{5} \Lambda_{-}(\mathbf{p}) \gamma_{5} \Lambda_{+}(\mathbf{p}) \gamma_{0}\right] & =2 M / E_{p}, \\
\operatorname{Tr}\left[\gamma_{5} \Lambda_{+}(\mathbf{p}) \gamma_{5} \Lambda_{-}(\mathbf{p}) \gamma_{0}\right] & =-2 M / E_{p}, \\
\operatorname{Tr}\left[\gamma_{5} \Lambda_{-}(\mathbf{p}) \gamma_{0} \Lambda_{+}(\mathbf{p}) \gamma_{5}\right] & =\operatorname{Tr}\left[\gamma_{5} \Lambda_{+}(\mathbf{p}) \gamma_{0} \Lambda_{-}(\mathbf{p}) \gamma_{5}\right] \\
& =0, \\
\operatorname{Tr}\left[\gamma_{5} \Lambda_{+}(\mathbf{p}) \gamma_{0} \Lambda_{+}(\mathbf{p}) \gamma_{5}\right] & =2 M / E_{p}, \\
\operatorname{Tr}\left[\gamma_{5} \Lambda_{-}(\mathbf{p}) \gamma_{0} \Lambda_{-}(\mathbf{p}) \gamma_{5}\right] & =-2 M / E_{p} .
\end{aligned}
$$

\section{APPENDIX B: POLARIZATION FUNCTIONS IN SU(2) NJL MODEL}

We list the meson polarization functions $\Pi_{i j}(k)$ in the $\mathrm{SU}(2)$ NJL model for $i, j=\sigma, \pi_{1}, \pi_{2}, \pi_{3}$ and for $i, j=$ $\sigma, \pi_{+}, \pi_{-}, \pi_{0}$. With respect to the basis $\left(\sigma, \pi_{1}, \pi_{2}, \pi_{3}\right)$, they are

$$
\begin{aligned}
\Pi_{00}(k) & =i N_{c} \int \frac{d^{4} p}{(2 \pi)^{4}} \operatorname{Tr}_{D}\left(\mathcal{S}_{u u}(q) \mathcal{S}_{u u}(p)+\mathcal{S}_{d d}(q) \mathcal{S}_{d d}(p)\right. \\
& \left.+\mathcal{S}_{u d}(q) \mathcal{S}_{d u}(p)+\mathcal{S}_{d u}(q) \mathcal{S}_{u d}(p)\right) \\
\Pi_{11}(k) & =-i N_{c} \int \frac{d^{4} p}{(2 \pi)^{4}} \operatorname{Tr}_{D}\left(\gamma_{5} \mathcal{S}_{u u}(q) \gamma_{5} \mathcal{S}_{d d}(p)\right. \\
& +\gamma_{5} \mathcal{S}_{d d}(q) \gamma_{5} \mathcal{S}_{u u}(p)+\gamma_{5} \mathcal{S}_{u d}(q) \gamma_{5} \mathcal{S}_{u d}(p) \\
& \left.+\gamma_{5} \mathcal{S}_{d u}(q) \gamma_{5} \mathcal{S}_{d u}(p)\right) \\
\Pi_{22}(k) & =-i N_{c} \int \frac{d^{4} p}{(2 \pi)^{4}} \operatorname{Tr}_{D}\left(\gamma_{5} \mathcal{S}_{u u}(q) \gamma_{5} \mathcal{S}_{d d}(p)\right. \\
& +\gamma_{5} \mathcal{S}_{d d}(q) \gamma_{5} \mathcal{S}_{u u}(p)-\gamma_{5} \mathcal{S}_{u d}(q) \gamma_{5} \mathcal{S}_{u d}(p) \\
& \left.-\gamma_{5} \mathcal{S}_{d u}(q) \gamma_{5} \mathcal{S}_{d u}(p)\right) \\
\Pi_{33}(k) & =-i N_{c} \int \frac{d^{4} p}{(2 \pi)^{4}} \operatorname{Tr}_{D}\left(\gamma_{5} \mathcal{S}_{u u}(q) \gamma_{5} \mathcal{S}_{u u}(p)\right. \\
& +\gamma_{5} \mathcal{S}_{d d}(q) \gamma_{5} \mathcal{S}_{d d}(p)-\gamma_{5} \mathcal{S}_{u d}(q) \gamma_{5} \mathcal{S}_{d u}(p) \\
& \left.-\gamma_{5} \mathcal{S}_{d u}(q) \gamma_{5} \mathcal{S}_{u d}(p)\right) \\
\Pi_{01}(k) & =-N_{c} \int \frac{d^{4} p}{(2 \pi)^{4}} \operatorname{Tr}_{D}\left(\mathcal{S}_{u u}(q) \gamma_{5} \mathcal{S}_{d u}(p)\right. \\
& +\mathcal{S}_{d d}(q) \gamma_{5} \mathcal{S}_{u d}(p)+\mathcal{S}_{u d}(q) \gamma_{5} \mathcal{S}_{u u}(p) \\
& \left.+\mathcal{S}_{d u}(q) \gamma_{5} \mathcal{S}_{d d}(p)\right) \\
& \left.+\gamma_{5} \mathcal{S}_{d u}(q) \mathcal{S}_{u u}(p)\right) \\
\Pi_{10}(k) & =-N_{c} \int \frac{d^{4} p}{(2 \pi)^{4}} \operatorname{Tr}_{D}\left(\gamma_{5} \mathcal{S}_{u u}(q) \mathcal{S}_{u d}(p)\right. \\
& \gamma_{5} \mathcal{S}_{d d}(q) \mathcal{S}_{d u}(p)+\gamma_{5} \mathcal{S}_{u d}(q) \mathcal{S}_{d d}(p) \\
&
\end{aligned}
$$

$$
\begin{aligned}
& \Pi_{02}(k)=i N_{c} \int \frac{d^{4} p}{(2 \pi)^{4}} \operatorname{Tr}_{D}\left(\mathcal{S}_{u u}(q) \gamma_{5} \mathcal{S}_{d u}(p)\right. \\
& -\mathcal{S}_{d d}(q) \gamma_{5} \mathcal{S}_{u d}(p)+\mathcal{S}_{d u}(q) \gamma_{5} \mathcal{S}_{d d}(p) \\
& \left.-\mathcal{S}_{u d}(q) \gamma_{5} \mathcal{S}_{u u}(p)\right) \text {, } \\
& \Pi_{20}(k)=i N_{c} \int \frac{d^{4} p}{(2 \pi)^{4}} \operatorname{Tr}_{D}\left(\gamma_{5} \mathcal{S}_{d d}(q) \mathcal{S}_{d u}(p)\right. \\
& -\gamma_{5} \mathcal{S}_{u u}(q) \mathcal{S}_{u d}(p)+\gamma_{5} \mathcal{S}_{d u}(q) \mathcal{S}_{u u}(p) \\
& \left.-\gamma_{5} \mathcal{S}_{u d}(q) \mathcal{S}_{d d}(p)\right) \text {, } \\
& \Pi_{03}(k)=N_{c} \int \frac{d^{4} p}{(2 \pi)^{4}} \operatorname{Tr}_{D}\left(\mathcal{S}_{d d}(q) \gamma_{5} \mathcal{S}_{d d}(p)\right. \\
& -\mathcal{S}_{u u}(q) \gamma_{5} \mathcal{S}_{u u}(p)+\mathcal{S}_{u d}(q) \gamma_{5} \mathcal{S}_{d u}(p) \\
& \left.-\mathcal{S}_{d u}(q) \gamma_{5} \mathcal{S}_{u d}(p)\right) \text {, } \\
& \Pi_{30}(k)=N_{c} \int \frac{d^{4} p}{(2 \pi)^{4}} \operatorname{Tr}_{D}\left(\gamma_{5} \mathcal{S}_{d d}(q) \mathcal{S}_{d d}(p)\right. \\
& -\gamma_{5} \mathcal{S}_{u u}(q) \mathcal{S}_{u u}(p)+\gamma_{5} \mathcal{S}_{d u}(q) \mathcal{S}_{u d}(p) \\
& \left.-\gamma_{5} \mathcal{S}_{u d}(q) \mathcal{S}_{d u}(p)\right) \\
& \Pi_{12}(k)=-N_{c} \int \frac{d^{4} p}{(2 \pi)^{4}} \operatorname{Tr}_{D}\left(\gamma_{5} \mathcal{S}_{u u}(q) \gamma_{5} \mathcal{S}_{d d}(p)\right. \\
& -\gamma_{5} \mathcal{S}_{d d}(q) \gamma_{5} \mathcal{S}_{u u}(p)+\gamma_{5} \mathcal{S}_{d u}(q) \gamma_{5} \mathcal{S}_{d u}(p) \\
& \left.-\gamma_{5} \mathcal{S}_{u d}(q) \gamma_{5} \mathcal{S}_{u d}(p)\right) \text {, } \\
& \Pi_{21}(k)=-N_{c} \int \frac{d^{4} p}{(2 \pi)^{4}} \operatorname{Tr}_{D}\left(\gamma_{5} \mathcal{S}_{d d}(q) \gamma_{5} \mathcal{S}_{u u}(p)\right. \\
& -\gamma_{5} \mathcal{S}_{u u}(q) \gamma_{5} \mathcal{S}_{d d}(p)+\gamma_{5} \mathcal{S}_{d u}(q) \gamma_{5} \mathcal{S}_{d u}(p) \\
& \left.-\gamma_{5} \mathcal{S}_{u d}(q) \gamma_{5} \mathcal{S}_{u d}(p)\right), \\
& \Pi_{13}(k)=i N_{c} \int \frac{d^{4} p}{(2 \pi)^{4}} \operatorname{Tr}_{D}\left(\gamma_{5} \mathcal{S}_{u u}(q) \gamma_{5} \mathcal{S}_{u d}(p)\right. \\
& -\gamma_{5} \mathcal{S}_{d d}(q) \gamma_{5} \mathcal{S}_{d u}(p)+\gamma_{5} \mathcal{S}_{d u}(q) \gamma_{5} \mathcal{S}_{u u}(p) \\
& \left.-\gamma_{5} \mathcal{S}_{u d}(q) \gamma_{5} \mathcal{S}_{d d}(p)\right), \\
& \Pi_{31}(k)=i N_{c} \int \frac{d^{4} p}{(2 \pi)^{4}} \operatorname{Tr}_{D}\left(\gamma_{5} \mathcal{S}_{u u}(q) \gamma_{5} \mathcal{S}_{d u}(p)\right. \\
& -\gamma_{5} \mathcal{S}_{d d}(q) \gamma_{5} \mathcal{S}_{u d}(p)+\gamma_{5} \mathcal{S}_{u d}(q) \gamma_{5} \mathcal{S}_{u u}(p) \\
& \left.-\gamma_{5} \mathcal{S}_{d u}(q) \gamma_{5} \mathcal{S}_{d d}(p)\right) \text {, } \\
& \Pi_{23}(k)=N_{c} \int \frac{d^{4} p}{(2 \pi)^{4}} \operatorname{Tr}_{D}\left(\gamma_{5} \mathcal{S}_{d u}(q) \gamma_{5} \mathcal{S}_{u u}(p)\right. \\
& +\gamma_{5} \mathcal{S}_{u d}(q) \gamma_{5} \mathcal{S}_{d d}(p)-\gamma_{5} \mathcal{S}_{u u}(q) \gamma_{5} \mathcal{S}_{u d}(p) \\
& \left.-\gamma_{5} \mathcal{S}_{d d}(q) \gamma_{5} \mathcal{S}_{d u}(p)\right) \text {, } \\
& \Pi_{32}(k)=N_{c} \int \frac{d^{4} p}{(2 \pi)^{4}} \operatorname{Tr}_{D}\left(\gamma_{5} \mathcal{S}_{u u}(q) \gamma_{5} \mathcal{S}_{d u}(p)\right. \\
& +\gamma_{5} \mathcal{S}_{d d}(q) \gamma_{5} \mathcal{S}_{u d}(p)-\gamma_{5} \mathcal{S}_{u d}(q) \gamma_{5} \mathcal{S}_{u u}(p) \\
& \left.-\gamma_{5} \mathcal{S}_{d u}(q) \gamma_{5} \mathcal{S}_{d d}(p)\right) \text {, }
\end{aligned}
$$

where the momentum $q$ is defined as $q=p+k$.

For determining the meson masses in RPA, we need 
only the polarization functions at $\mathbf{k}=0$. With the calculated trace shown in Appendix 1, we have

$$
\Pi_{03}=\Pi_{30}=\Pi_{13}=\Pi_{31}=\Pi_{23}=\Pi_{32}=0 .
$$

If we further set $k_{0}=0$ which is used to determine the temperature and chemical potentials corresponding to the zero meson mass, we obtain

$$
\Pi_{02}=\Pi_{20}=\Pi_{12}=\Pi_{21}=0 .
$$

After performing the Matsubara frequency summation we derive the expressions as explicit functions of temperature and chemical potentials,

$$
\begin{aligned}
& \Pi_{22}\left(k_{0}=0\right)=-2 N_{c} \int \frac{d^{3} \mathbf{p}}{(2 \pi)^{3}}\left[\frac{1}{E_{p}^{-}}\left(f\left(E_{-}^{-}\right)-f\left(-E_{+}^{-}\right)\right)\right. \\
& \left.+\frac{1}{E_{p}^{+}}\left(f\left(E_{-}^{+}\right)-f\left(-E_{+}^{+}\right)\right)\right], \\
& \Pi_{33}\left(k_{0}\right)=2 N_{c} \int \frac{d^{3} \mathbf{p}}{(2 \pi)^{3}} \\
& {\left[\frac{E_{p}^{-} E_{p}^{+}-\left(E_{p}^{2}-\mu_{I}^{2} / 4\right)-4 G^{2} \pi^{2}}{k_{0}^{2}-\left(E_{p}^{-}-E_{p}^{+}\right)^{2}}\left(\frac{1}{E_{p}^{+}}-\frac{1}{E_{p}^{-}}\right)\right.} \\
& +\frac{E_{p}^{-} E_{p}^{+}+\left(E_{p}^{-}-\mu_{I}^{2} / 4\right)+4 G^{2} \pi^{2}}{k_{0}^{2}-\left(E_{p}^{-}+E_{p}^{+}\right)^{2}}\left(\frac{1}{E_{p}^{+}}+\frac{1}{E_{p}^{-}}\right) \\
& \left.\left(f\left(E_{-}^{-}\right)+f\left(E_{-}^{+}\right)-f\left(-E_{+}^{-}\right)-f\left(-E_{+}^{+}\right)\right)\right] .
\end{aligned}
$$

The meson polarization functions in the basis $\left(\sigma, \pi_{+}, \pi_{-}, \pi_{0}\right)$ are defined as

$$
\begin{aligned}
\Pi_{\sigma \sigma}(k) & =i N_{c} \int \frac{d^{4} p}{(2 \pi)^{4}} \operatorname{Tr}_{D}\left(\mathcal{S}_{u u}(q) \mathcal{S}_{u u}(p)+\mathcal{S}_{d d}(q) \mathcal{S}_{d d}(p)\right. \\
& \left.+\mathcal{S}_{u d}(q) \mathcal{S}_{d u}(p)+\mathcal{S}_{d u}(q) \mathcal{S}_{u d}(p)\right) \\
\Pi_{\pi_{+} \pi_{+}}(k) & =-2 i N_{c} \int \frac{d^{4} p}{(2 \pi)^{4}} \operatorname{Tr}_{D}\left(\gamma_{5} \mathcal{S}_{u u}(p+k) \gamma_{5} \mathcal{S}_{d d}(p)\right) \\
\Pi_{\pi_{-} \pi_{-}}(k) & =-2 i N_{c} \int \frac{d^{4} p}{(2 \pi)^{4}} \operatorname{Tr}_{D}\left(\gamma_{5} \mathcal{S}_{d d}(p+k) \gamma_{5} \mathcal{S}_{u u}(p)\right) \\
\Pi_{\pi_{+} \pi_{-}}(k) & =-2 i N_{c} \int \frac{d^{4} p}{(2 \pi)^{4}} \operatorname{Tr}_{D}\left(\gamma_{5} \mathcal{S}_{u d}(p+k) \gamma_{5} \mathcal{S}_{u d}(p)\right) \\
\Pi_{\pi_{-} \pi_{+}}(k) & =-2 i N_{c} \int \frac{d^{4} p}{(2 \pi)^{4}} \operatorname{Tr}_{D}\left(\gamma_{5} \mathcal{S}_{d u}(p+k) \gamma_{5} \mathcal{S}_{d u}(p)\right) \\
\Pi_{\sigma \pi_{+}}(k) & =-\sqrt{2} N_{c} \int \frac{d^{4} p}{(2 \pi)^{4}} \operatorname{Tr}_{D}\left(\mathcal{S}_{u u}(p+k) \gamma_{5} \mathcal{S}_{d u}(p)\right. \\
& \left.+\mathcal{S}_{d u}(p+k) \gamma_{5} \mathcal{S}_{d d}(p)\right) \\
\Pi_{\sigma \pi_{-}}(k) & =-\sqrt{2} N_{c} \int \frac{d^{4} p}{(2 \pi)^{4}} \operatorname{Tr}_{D}\left(\mathcal{S}_{u d}(p+k) \gamma_{5} \mathcal{S}_{u u}(p)\right. \\
& \left.+\mathcal{S}_{d d}(p+k) \gamma_{5} \mathcal{S}_{u d}(p)\right)
\end{aligned}
$$

$$
\begin{aligned}
\Pi_{\pi_{+} \sigma}(k) & =-\sqrt{2} N_{c} \int \frac{d^{4} p}{(2 \pi)^{4}} \operatorname{Tr}_{D}\left(\gamma_{5} \mathcal{S}_{u u}(p+k) \mathcal{S}_{u d}(p)\right. \\
& \left.+\gamma_{5} \mathcal{S}_{u d}(p+k) \mathcal{S}_{d d}(p)\right) \\
\Pi_{\pi_{-} \sigma}(k) & =-\sqrt{2} N_{c} \int \frac{d^{4} p}{(2 \pi)^{4}} \operatorname{Tr}_{D}\left(\gamma_{5} \mathcal{S}_{d u}(p+k) \mathcal{S}_{u u}(p)\right. \\
& \left.+\gamma_{5} \mathcal{S}_{d d}(p+k) \mathcal{S}_{d u}(p)\right)
\end{aligned}
$$

The explicit expressions as functions of temperature and chemical potentials at $\mathbf{k}=0$ can be written as

$$
\begin{aligned}
& \Pi_{\sigma \sigma}\left(k_{0}\right)=2 N_{c} \int \frac{d^{3} \mathbf{p}}{(2 \pi)^{3}} \frac{\mathbf{p}^{2}}{E_{p}^{2}} \\
& {\left[\frac{E_{p}^{-} E_{p}^{+}-\left(E_{p}^{2}-\mu_{I}^{2} / 4\right)-4 G^{2} \pi^{2}}{k_{0}^{2}-\left(E_{p}^{-}-E_{p}^{+}\right)^{2}}\left(\frac{1}{E_{p}^{+}}-\frac{1}{E_{p}^{-}}\right)\right.} \\
& \left(f\left(E_{-}^{-}\right)+f\left(-E_{+}^{+}\right)-f\left(-E_{+}^{-}\right)-f\left(E_{-}^{+}\right)\right) \\
& +\frac{E_{p}^{-} E_{p}^{+}+\left(E_{p}^{2}-\mu_{I}^{2} / 4\right)+4 G^{2} \pi^{2}}{k_{0}^{2}-\left(E_{p}^{-}+E_{p}^{+}\right)^{2}}\left(\frac{1}{E_{p}^{+}}+\frac{1}{E_{p}^{-}}\right) \\
& \left.\left.\left(f\left(E_{-}^{-}\right)+f\left(E_{-}^{+}\right)-f\left(-E_{+}^{-}\right)-f_{(}-E_{+}^{+}\right)\right)\right], \\
& +4 N_{c} \int \frac{d^{3} \mathbf{p}}{(2 \pi)^{3}} \frac{M_{q}^{2}}{E_{p}^{2}} \\
& {\left[\frac{4 G^{2} \pi^{2}}{k_{0}^{2}-4\left(E_{p}^{-}\right)^{2}} \frac{1}{E_{p}^{-}}\left(f\left(E_{-}^{-}\right)-f\left(-E_{+}^{-}\right)\right)\right.} \\
& \left.+\frac{4 G^{2} \pi^{2}}{k_{0}^{2}-4\left(E_{p}^{+}\right)^{2}} \frac{1}{E_{p}^{+}}\left(f\left(E_{-}^{+}\right)-f\left(-E_{+}^{+}\right)\right)\right], \\
& \Pi_{\pi_{+} \pi_{+}}\left(k_{0}\right)=4 N_{c} \int \frac{d^{3} \mathbf{p}}{(2 \pi)^{3}} \\
& {\left[\frac{\left(E_{p}^{-}\right)^{2}+\left(E_{p}-\mu_{I} / 2\right)^{2}+k_{0}\left(E_{p}-\mu_{I} / 2\right)}{k_{0}^{2}-4\left(E_{p}^{-}\right)^{2}}\right.} \\
& \frac{1}{E_{p}^{-}}\left(f\left(E_{-}^{-}\right)-f\left(-E_{+}^{-}\right)\right) \\
& +\frac{\left(E_{p}^{+}\right)^{2}+\left(E_{p}+\mu_{I} / 2\right)^{2}-k_{0}\left(E_{p}+\mu_{I} / 2\right)}{k_{0}^{2}-4\left(E_{p}^{+}\right)^{2}} \\
& \left.\frac{1}{E_{p}^{+}}\left(f\left(E_{-}^{+}\right)-f\left(-E_{+}^{+}\right)\right)\right] \text {, } \\
& \Pi_{\pi_{-} \pi_{-}}\left(k_{0}\right)=4 N_{c} \int \frac{d^{3} \mathbf{p}}{(2 \pi)^{3}} \\
& {\left[\frac{\left(E_{p}^{-}\right)^{2}+\left(E_{p}-\mu_{I} / 2\right)^{2}-k_{0}\left(E_{p}-\mu_{I} / 2\right)}{k_{0}^{2}-4\left(E_{p}^{-}\right)^{2}}\right.} \\
& \frac{1}{E_{p}^{-}}\left(f\left(E_{-}^{-}\right)-f\left(-E_{+}^{-}\right)\right) \\
& +\frac{\left(E_{p}^{+}\right)^{2}+\left(E_{p}+\mu_{I} / 2\right)^{2}+k_{0}\left(E_{p}+\mu_{I} / 2\right)}{k_{0}^{2}-4\left(E_{p}^{+}\right)^{2}}
\end{aligned}
$$




$$
\begin{aligned}
& \left.\quad \frac{1}{E_{p}^{+}}\left(f\left(E_{+}^{-}\right)-f\left(-E_{+}^{+}\right)\right)\right], \\
& \Pi_{\pi_{+} \pi_{-}}\left(k_{0}\right)=\Pi_{\pi_{-} \pi_{+}}\left(k_{0}\right)=-4 N_{c} \int \frac{d^{3} \mathbf{p}}{(2 \pi)^{3}} \\
& {\left[\frac{4 G^{2} \pi^{2}}{k_{0}^{2}-4\left(E_{p}^{-}\right)^{2}} \frac{1}{E_{p}^{-}}\left(f\left(E_{-}^{-}\right)-f\left(-E_{+}^{-}\right)\right)\right.} \\
& \left.+\frac{4 G^{2} \pi^{2}}{k_{0}^{2}-4\left(E_{p}^{+}\right)^{2}} \frac{1}{E_{p}^{+}}\left(f\left(E_{-}^{+}\right)-f\left(-E_{+}^{+}\right)\right)\right], \\
& \Pi_{\sigma \pi_{+}}\left(k_{0}\right)=\Pi_{\pi_{+} \sigma}\left(k_{0}\right)=4 \sqrt{2} N_{c} G \pi \int \frac{d^{3} \mathbf{p}}{(2 \pi)^{3}} \frac{M_{q}}{E_{p}} \\
& {\left[\frac{2 E_{p}-\mu_{I}+k_{0}}{k_{0}^{2}-4\left(E_{p}^{-}\right)^{2}} \frac{1}{E_{p}^{-}}\left(f\left(E_{-}^{-}\right)-f\left(-E_{+}^{-}\right)\right)\right.} \\
& \left.+\frac{2 E_{p}+\mu_{I}-k_{0}}{k_{0}^{2}-4\left(E_{p}^{+}\right)^{2}} \frac{1}{E_{p}^{+}}\left(f\left(E_{-}^{+}\right)-f\left(-E_{+}^{+}\right)\right)\right] \\
& \Pi_{\sigma \pi_{-}}\left(k_{0}\right)=\Pi_{\pi_{-} \sigma}\left(k_{0}\right)=4 \sqrt{2} N_{c} G \pi \int \frac{d^{3} \mathbf{p}}{(2 \pi)^{3}} \frac{M_{q}}{E_{p}} \\
& \quad\left[\frac{2 E_{p}-\mu_{I}-k_{0}}{k_{0}^{2}-4\left(E_{p}^{-}\right)^{2}} \frac{1}{E_{p}^{-}}\left(f\left(E_{-}^{-}\right)-f\left(-E_{+}^{-}\right)\right)\right. \\
& \left.+\frac{2 E_{p}+\mu_{I}+k_{0}}{k_{0}^{2}-4\left(E_{p}^{+}\right)^{2}} \frac{1}{E_{p}^{+}}\left(f\left(E_{-}^{+}\right)-f\left(-E_{+}^{+}\right)\right)\right] .
\end{aligned}
$$

where the thermal excitation functions $\langle\sigma \sigma\rangle,\langle\pi \pi\rangle$ and $\left\langle\pi_{0} \pi_{0}\right\rangle$ are calculated from the mean field propagators,

$$
\begin{aligned}
\langle\sigma \sigma\rangle= & \int \frac{d^{3} \mathbf{k}}{(2 \pi)^{3}} \frac{1}{\sqrt{k^{2}+M_{\sigma}^{2}}} \frac{1}{e^{\sqrt{\mathbf{k}^{2}+\mathbf{M}_{\sigma}^{2}} / T}-1}, \\
\left\langle\pi_{0} \pi_{0}\right\rangle= & \int \frac{d^{3} \mathbf{k}}{(2 \pi)^{3}} \frac{1}{\sqrt{k^{2}+M_{0}^{2}}} \frac{1}{e^{\sqrt{\mathbf{k}^{2}+\mathbf{M}_{\mathbf{0}}^{2}} / T}-1}, \\
\langle\pi \pi\rangle= & \int \frac{d^{3} \mathbf{k}}{(2 \pi)^{3}} \frac{1}{2 \sqrt{k^{2}+M_{\pi}^{2}}} \\
& {\left[\frac{1}{e^{\left(\sqrt{\mathbf{k}^{2}+\mathbf{M}_{\pi}^{2}}-\mu_{I}\right) / T}-1}+\frac{1}{e^{\left(\sqrt{\mathbf{k}^{2}+\mathbf{M}_{\pi}^{2}}+\mu_{I}\right) / T}-1}\right] . }
\end{aligned}
$$

The thermodynamic potential of the system in HartreeFock approximation can be expressed as the summation of the quasi-particle contributions plus the effective potential,

$$
\begin{aligned}
\Omega & =\bar{U}(\xi)+T \int \frac{d^{3} \mathbf{k}}{\left(2 \pi^{3}\right)} \ln \left[1-e^{-\sqrt{\mathbf{k}^{2}+\mathbf{M}_{\sigma}^{2}} / T}\right] \\
& +T \int \frac{d^{3} \mathbf{k}}{\left(2 \pi^{3}\right)} \ln \left[1-e^{-\sqrt{\mathbf{k}^{2}+\mathbf{M}_{\mathbf{0}}^{2}} / T}\right] \\
& +T \int \frac{d^{3} \mathbf{k}}{\left(2 \pi^{3}\right)} \ln \left[1-e^{-\left(\sqrt{\mathbf{k}^{2}+\mathbf{M}_{\pi}^{2}}-\mu_{I}\right) / T}\right] \\
& +T \int \frac{d^{3} \mathbf{k}}{\left(2 \pi^{3}\right)} \ln \left[1-e^{-\left(\sqrt{\mathbf{k}^{2}+\mathbf{M}_{\pi}^{2}}+\mu_{I}\right) / T}\right] .
\end{aligned}
$$

The gap equation for the chiral condensate $\xi$ is derived by minimizing the thermodynamic potential,

$2 g_{\pi}^{2} \xi\left(\xi^{2}-f_{\pi}^{2}+3\langle\sigma \sigma\rangle+2\langle\pi \pi\rangle+\left\langle\pi_{0} \pi_{0}\right\rangle\right)+\xi m_{\pi}^{2}=f_{\pi} m_{\pi}^{2}$.

The mass equations (C2) with the thermal excitation functions (C4) and the gap equation (C6) form a group of coupled equations and determine self-consistently the temperature and chemical potential behavior of the chiral condensate and the effective meson masses.

\section{APPENDIX D: POLARIZATION FUNCTIONS IN \\ $S U(3)$ NJL MODEL}

$$
\begin{aligned}
& M_{\sigma}^{2}=2 g_{\pi}^{2}\left(3 \xi^{2}-f_{\pi}^{2}+3\langle\sigma \sigma\rangle+2\langle\pi \pi\rangle+\left\langle\pi_{0} \pi_{0}\right\rangle\right)+m_{\pi}^{2}, \\
& M_{0}^{2}=2 g_{\pi}^{2}\left(\xi^{2}-f_{\pi}^{2}+\langle\sigma \sigma\rangle+2\langle\pi \pi\rangle+3\left\langle\pi_{0} \pi_{0}\right\rangle\right)+m_{\pi}^{2}, \\
& M_{\pi}^{2}=2 g_{\pi}^{2}\left(\xi^{2}-f_{\pi}^{2}+\langle\sigma \sigma\rangle+4\langle\pi \pi\rangle+\left\langle\pi_{0} \pi_{0}\right\rangle\right)+m_{\pi}^{2},
\end{aligned}
$$

We define the following matrices

$$
\lambda_{a b}^{ \pm}=\frac{1}{\sqrt{2}}\left(\lambda_{a} \pm i \lambda_{b}\right) .
$$


The meson polarization functions are defined as

$$
\Pi_{M M}(k)=i \int \frac{d^{4} p}{(2 \pi)^{4}} \operatorname{Tr}\left(\Gamma_{M}^{*} \mathcal{S}_{m f}(p+k) \Gamma_{M} \mathcal{S}_{m f}(p)\right)
$$

with the couplings

$$
\Gamma_{M}= \begin{cases}\lambda_{12}^{+} & M=a^{+} \\ \lambda_{12}^{-} & M=a^{-} \\ \lambda_{3} & M=a^{0} \\ \lambda_{45}^{+} & M=\kappa^{+} \\ \lambda_{45}^{-} & M=\kappa^{-} \\ \lambda_{67}^{+} & M=\kappa^{0} \\ \lambda_{67}^{-} & M=\bar{\kappa}^{0} \\ i \gamma_{5} \lambda_{12}^{+} & M=\pi^{+} \\ i \gamma_{5} \lambda_{12}^{-} & M=\pi^{-} \\ i \gamma_{5} \lambda_{3} & M=\pi^{0} \\ i \gamma_{5} \lambda_{45}^{+} & M=K^{+} \\ i \gamma_{5} \lambda_{45}^{-} & M=K^{-} \\ i \gamma_{5} \lambda_{67}^{+} & M=K^{0} \\ i \gamma_{5} \lambda_{67}^{-} & M=\bar{K}^{0} \\ \Gamma_{M}^{-} & M=a^{+} \\ \lambda_{12}^{+} & M=a^{-} \\ \lambda_{3} & M=a^{0} \\ \lambda_{45}^{-} & M=\kappa^{+} \\ \lambda_{45}^{+} & M=\kappa^{-} \\ \lambda_{67}^{-} & M=\kappa^{0} \\ \lambda_{67}^{+} & M=\bar{\kappa}^{0} \\ i \gamma_{5} \lambda_{12}^{-} & M=\pi^{+} \\ i \gamma_{5} \lambda_{12}^{+} & M=\pi^{-} \\ i \gamma_{5} \lambda_{3} & M=\pi^{0} \\ i \gamma_{5} \lambda_{45}^{-} & M=K^{+} \\ i \gamma_{5} \lambda_{45}^{+} & M=K^{-} \\ i \gamma_{5} \lambda_{67}^{-} & M=K^{0} \\ i \gamma_{5} \lambda_{67}^{+} & M=\bar{K}^{0}\end{cases}
$$

Doing the trace in color and flavor space first, we obtain

$$
\begin{aligned}
\Pi_{a_{0} a_{0}}(k) & =i N_{c} \int \frac{d^{4} p}{(2 \pi)^{4}} \operatorname{Tr}_{D}\left(\mathcal{S}_{u}(p+k) \mathcal{S}_{u}(p)\right. \\
& \left.+\mathcal{S}_{d}(p+k) \mathcal{S}_{d}(p)\right), \\
\Pi_{a_{+} a_{+}}(k) & =2 i N_{c} \int \frac{d^{4} p}{(2 \pi)^{4}} \operatorname{Tr}_{D}\left(\mathcal{S}_{u}(p+k) \mathcal{S}_{d}(p)\right), \\
\Pi_{a_{-} a_{-}}(k) & =6 i \int \frac{d^{4} p}{(2 \pi)^{4}} \operatorname{Tr}_{D}\left(\mathcal{S}_{d}(p+k) \mathcal{S}_{u}(p)\right), \\
\Pi_{k_{+} k_{+}}(k) & =2 N_{c} i \int \frac{d^{4} p}{(2 \pi)^{4}} \operatorname{Tr}_{D}\left(\mathcal{S}_{u}(p+k) \mathcal{S}_{s}(p)\right), \\
\Pi_{k_{-} k_{-}}(k) & =2 N_{c} i \int \frac{d^{4} p}{(2 \pi)^{4}} \operatorname{Tr}_{D}\left(\mathcal{S}_{s}(p+k) \mathcal{S}_{u}(p)\right), \\
\Pi_{k^{0} k^{0}}(k) & =2 N_{c} i \int \frac{d^{4} p}{(2 \pi)^{4}} \operatorname{Tr}_{D}\left(\mathcal{S}_{d}(p+k) \mathcal{S}_{s}(p)\right), \\
\Pi_{\bar{k}^{0} \bar{k}^{0}}(k) & =2 N_{c} i \int \frac{d^{4} p}{(2 \pi)^{4}} \operatorname{Tr}_{D}\left(\mathcal{S}_{s}(p+k) \mathcal{S}_{d}(p)\right),
\end{aligned}
$$

$$
\begin{aligned}
\Pi_{\pi_{0} \pi_{0}}(k) & =-N_{c} i \int \frac{d^{4} p}{(2 \pi)^{4}} \operatorname{Tr}_{D}\left(\gamma_{5} \mathcal{S}_{u}(p+k) \gamma_{5} \mathcal{S}_{u}(p)\right. \\
& \left.+\gamma_{5} \mathcal{S}_{d}(p+k) \gamma_{5} \mathcal{S}_{d}(p)\right), \\
\Pi_{\pi_{+} \pi_{+}}(k) & =-2 N_{c} i \int \frac{d^{4} p}{(2 \pi)^{4}} \operatorname{Tr}_{D}\left(\gamma_{5} \mathcal{S}_{u}(p+k) \gamma_{5} \mathcal{S}_{d}(p)\right), \\
\Pi_{\pi_{-} \pi_{-}}(k) & =-2 N_{c} i \int \frac{d^{4} p}{(2 \pi)^{4}} \operatorname{Tr}_{D}\left(\gamma_{5} \mathcal{S}_{d}(p+k) \gamma_{5} \mathcal{S}_{u}(p)\right), \\
\Pi_{K_{+} K_{+}}(k) & =-2 N_{c} i \int \frac{d^{4} p}{(2 \pi)^{4}} \operatorname{Tr}_{D}\left(\gamma_{5} \mathcal{S}_{u}(p+k) \gamma_{5} \mathcal{S}_{s}(p)\right), \\
\Pi_{K_{-} K_{-}}(k) & =-2 N_{c} i \int \frac{d^{4} p}{(2 \pi)^{4}} \operatorname{Tr}_{D}\left(\gamma_{5} \mathcal{S}_{s}(p+k) \gamma_{5} \mathcal{S}_{u}(p)\right), \\
\Pi_{K^{0} K^{0}}(k) & =-2 N_{c} i \int \frac{d^{4} p}{(2 \pi)^{4}} \operatorname{Tr}_{D}\left(\gamma_{5} \mathcal{S}_{d}(p+k) \gamma_{5} \mathcal{S}_{s}(p)\right), \\
\Pi_{\bar{K}^{0} \bar{K}^{0}}(k) & =-2 N_{c} i \int \frac{d^{4} p}{(2 \pi)^{4}} \operatorname{Tr}_{D}\left(\gamma_{5} \mathcal{S}_{s}(p+k) \gamma_{5} \mathcal{S}_{d}(p)\right) .
\end{aligned}
$$

[1] For instance, see Quark-Gluon Plasma, ed. R.C.Hwa (world Scientific, Singapore, 1990).

[2] M.Alford, K.Rajagopal, and F.Wilczek, Phys. Lett. B422, 247(1998); R.Rapp, T.Schäfer, E.V.Shuryak, and M.Velkovsky, Phys. Rev. Lett. 81, 53(1998).

[3]

A.B.Migdal,Zh.Eksp.Teor.Fiz.61,2210(1971)(Sov.Phys.JETP 36, 1052(1973))

[4] R.F.Sawyer,

Phys.Rev.Lett.29,382(1972); D.J.Scalapino,Phys.Rev.Lett.29,386(1972)

[5] D.K.Campbell,R.F.Dashen and J.T.Manassah, Phys.Rev.D12,979(1975);D12,1010(1975)

[6] D.B.Kaplan and A.E.Nelson, Phys.Lett.B175,57(1986)

[7] F.karsch, Lect. Notes Phys. 583, 209(2002).

[8] D.T.Son and M.A.Stephanov, Phys.Rev.Lett.86,592(2001);Phys.Atom.Nucl.64,834(2001)

[9] J.B.Kogut,D.K.Sinclair, Phys.Rev.D66, 034505(2002)

[10] J.B.Kogut,D.K.Sinclair, Phys.Rev.D66, 014508(2002)

[11] J.B.Kogut,D.K.Sinclair, Phys.Rev.D70, 094501(2004)

[12] J.B.Kogut and D.Toublan, Phys.Rev.D64, 034007(2001)

[13] K.Splittorff, D.T.Son and M.A.Stephanov, Phys.Rev. D64 016003(2001)

[14] M.Loewe and C.Villavicencio, Phys.Rev. D67, 074034(2003); Phys.Rev.D70, 074005(2004)

[15] Michael C. Birse, Thomas D. Cohen and Judith A. McGovern, Phys.Lett. B516 27(2001)

[16] D.Toublan and J.B.Kogut, Phys.Lett. B564, 212(2003)

[17] A.Barducci, R.Casalbuoni, G.Pettini, L.Ravagli, Phys.Rev. D69, 096004(2004)

[18] A.Barducci, R.Casalbuoni, G.Pettini, L.Ravagli, Phys.Rev. D71, 016011(2005)

[19] M.Frank, M.Buballa and M.Oertel, Phys.Lett. B562, $221(2003)$ 
[20] L.He and P.Zhuang, hep-ph/0501024, Phys. lett. B (in press)

[21] B.Klein, D.Toublan and J.J.M.Verbaarschot, hepph/0405180

[22] B.Klein, D.Toublan and J.J.M.Verbaarschot, Phys.Rev. D68, 014009(2003)

[23] A.Barducci, R.Casalbuoni, G.Pettini, L.Ravagli, Phys.Lett. B564, 217(2003)

[24] Y.Nishida, Phys.Rev.D69, 094501(2004)

[25] Nambu and G.Jona-Lasinio, Phys.Rev.122, 345(1961); 124, 246(1961)

[26] U.Vogl and Weise, Prog. Part. and Nucl. Phys. 27, 195(1991)

[27] S.P.Klevansky, Rev.Mod.Phys.64, 649(1992)

[28] M.K.Volkov, Phys.Part.Nucl.24, 35(1993)

[29] T.Hatsuda and T.Kunihiro, Phys.Rep.247, 221(1994)

[30] J.Hufner, S.P.Klevansky, P.Zhuang and H.Voss, Ann.Phys(N.Y)234, 225(1994)

[31] P.Zhuang, J.Hufner, S.P.Klevansky, Nucl.Phys.A576,525(1994)

[32] P.Zhuang and Z.Yang, Phys.Rev.C62, 054901(2000)

[33] T.M.Schwarz,S.P.Klevansky and G.Rapp, Phys.Rev.C60, 055205(1999)

[34] M.Huang,P.Zhuang and W.Chao, Phys.Rev.D65, 076012(2002)

[35] M.Huang,P.Zhuang and W.Chao, Phys.Rev.D67, 065015(2003)

[36] I.Shovkovy and M.Huang, Phys.Lett.B564, 205(2003)

[37] J.Liao and P.Zhuang, Phys.Rev.D68, 114016(2003)

[38] D.Ebert, K.G.Klimenko, V.L.Yudichev, hep-ph/0412129

[39] S.B.Ruster, V.Werth, M.Buballa, I.A.Shovkovy, D.H.Rischke, hep-ph/0503184

[40] D.Blaschke, S.Fredriksson, H.Grigorian, A.M.Oztas, F.Sandin, hep-ph/0503194

[41] V.Bernard, R.L.Jaffe and U.-G.Meissner, Nucl. Phys. B308, 753(1988)

[42] R.Rapp, T.Schäfer, E.V. Shuryak and M. Velkovsky, Phys.Rev.Lett.81, 53(1998)

[43] T.Eguchi and H.Sugawara, Phys.Rev.D10, 4257(1974);T.Eguchi, Phys.Rev.D14, 2755(1976)

[44] N.Petropoulos, hep-ph/0402136

[45] H.E.Haber and H.Arthur.Weldon, Phys.Rev.D25, 502(1982)

[46] P.Rehberg, S.P.Klevansky and J.Hüfner, Phys.Rev.C53, 410(1996)

[47] Z.Fodor and S.D.Katz, Nucl. Phys. B106 (Proc. Suppl.), 441(2002) 